\title{
LOXL2-mediated H3K4 oxidation reduces chromatin accessibility in triple-negative breast cancer cells
}

\author{
J. P. Cebrià-Costa ${ }^{1}$ - L. Pascual-Reguant ${ }^{1} \cdot$ A. Gonzalez-Perez ${ }^{2} \cdot$ G. Serra-Bardenys ${ }^{1} \cdot$ J. Querol $^{1} \cdot$ M. Cosín $^{1}$ \\ G. Verde $\mathbb{1}^{1,3} \cdot$ R. A. Cigliano ${ }^{4}$-W. Sanseverino $\mathbb{1}^{4} \cdot$ S. Segura-Bayona $\mathbb{D}^{2} \cdot$ A. Iturbide ${ }^{5} \cdot$ D. Andreu $\mathbb{D}^{6}$. \\ P. Nuciforo ${ }^{1}{ }^{1} \cdot$ C. Bernado-Morales ${ }^{1,7} \cdot$ V. Rodilla ${ }^{1} \cdot$ J. Arribas ${ }^{1,7,8,9} \cdot$ J. Yelamos $^{10} \cdot$ A. Garcia de Herreros ${ }^{6,10}$. \\ T. H. Stracker ${ }^{2} \cdot$ S. Peiró ${ }^{1}$
}

Received: 28 January 2019 / Revised: 8 July 2019 / Accepted: 9 August 2019 / Published online: 28 August 2019

(c) The Author(s) 2019. This article is published with open access

\begin{abstract}
Oxidation of $\mathrm{H} 3$ at lysine 4 (H3K4ox) by lysyl oxidase-like 2 (LOXL2) generates an H3 modification with an unknown physiological function. We find that LOXL2 and H3K4ox are higher in triple-negative breast cancer (TNBC) cell lines and patient-derived xenografts (PDXs) than those from other breast cancer subtypes. ChIP-seq revealed that H3K4ox is located primarily in heterochromatin, where it is involved in chromatin compaction. Knocking down LOXL2 reduces H3K4ox levels and causes chromatin decompaction, resulting in a sustained activation of the DNA damage response (DDR) and increased susceptibility to anticancer agents. This critical role that LOXL2 and oxidized H3 play in chromatin compaction and DDR suggests that functionally targeting LOXL2 could be a way to sensitize TNBC cells to conventional therapy.
\end{abstract}

Supplementary information The online version of this article (https:// doi.org/10.1038/s41388-019-0969-1) contains supplementary material, which is available to authorized users.

\section{S. Peiró}

speiro@vhio.net

$1 \quad$ Vall d'Hebron Institute of Oncology (VHIO), 08035 Barcelona, Spain

2 Institute for Research in Biomedicine (IRB Barcelona), Barcelona Institute of Science and Technology, 08028 Barcelona, Spain

3 Faculty of Medicine and Health Sciences, Universitat Internacional de Catalunya, Barcelona, Spain

4 Sequentia Biotech SL, Comte d'Urgell, 240, Barcelona, Spain

5 Institute of Epigenetics and Stem Cells, Helmoholtz Zentrum München, D-81377 München, Germany

6 Departament de Ciències Experimentals i de la Salut, Universitat Pompeu Fabra, Barcelona, Spain

7 Centro de Investigación Biomédica en Red en Oncología (CIBERONC), 08035 Barcelona, Spain

8 Institució Catalana de Recerca I Estudis Avançats (ICREA), Barcelona, Spain

9 Departament de Bioquímica y Biología Molecular, Universitat Autónoma de Barcelona, Bellaterra, Spain

10 Programa de Recerca en Càncer, Institut Hospital del Mar d'Investigacions Mèdiques (IMIM), Barcelona, Spain

\section{Introduction}

Histone modifications contribute to gene regulation both by directly affecting chromatin structure and by recruiting effector proteins [1]. Deregulation of this enzymatic system can contribute to diseases, including cancer. The lysyl oxidase family of proteins are copper- and quinonedependent amine oxidases that oxidize the amino group located in the epsilon-position in lysines, thereby generating an aldehyde group [2]. One of the members of the LOX family, lysyl oxidase-like 2 (LOXL2), deaminates unmethylated and trimethylated lysine 4 in histone $\mathrm{H} 3$ (H3K4me3) through an amino-oxidase reaction that uses the $\mathrm{Cu}(\mathrm{II})$ ion and the internal cofactor lysine-tyrosylquinone, releasing the amino group and converting $\mathrm{K} 4$ into an allysine (H3K4ox) [3, 4]. Generation of this peptidyl aldehyde likely alters the local macromolecular structure of chromatin and the nature of any protein-protein or protein-nucleic acid interactions. This is particularly relevant for gene regulation, as changes in the macromolecular status of histones can affect chromatin conformation [4-6].

LOXL2 is overexpressed in many tumors, and especially in breast cancers [7-9]. In this light, it is intriguing that some breast cancers are intrinsically resistant to chemotherapy; for these subtypes, chemotherapy induces a mesenchymal phenotype through the epithelial-to-mesenchymal transition 
(EMT) [10]. EMT is likely to be a critical switch for tumor cell invasiveness and cell death resistance [11-13] and to involve chromatin reorganization, as it requires dramatic changes in cellular characteristics and gene expression $[6,14]$. Notably, the key transcription factor Snaill interacts with LOXL2 [15], and LOXL2 H3K4 oxidase activity generates an $\mathrm{H} 3 \mathrm{~K} 4$ ox that regulates the repression of the Ecadherin gene $(\mathrm{CDH1})$ and heterochromatin transcription, which play roles in two essential steps of EMT $[6,16]$.

Double-strand breaks (DSBs) are a major form of DNA damage and cause a specific signaling response, the DNA damage response (DDR), which can activate cell cycle checkpoint arrest and cell fate decisions, such as apoptosis or senescence. One of the first steps of DDR is the accumulation of DNA repair proteins at the damaged site [17]. Importantly, DDR activation can occur in the absence of DNA damage, by stable association of the repair factors with chromatin [18, 19]. Moreover, increasing evidence suggests that higher-order chromatin structures affect DSB repair and signaling [20]. For example, DDR actively regulates decondensation of chromatin after DSBs [21], and it is amplified when chromatin is in an "open" state [20]. Similarly, DDR signaling is affected by chromatin compaction in a DNA damage-independent manner [18, 19, 22-24].

We addressed the physiological functions of H3K4ox using an in-house generated antibody specific for this modification to analyze the H3K4ox levels in distinct breast cancer subtypes. Intriguingly, mesenchymal triple-negative breast cancer (TNBC) cell lines as well as breast cancer patient-derived xenografts (PDXs) had high H3K4ox levels that correlated with high LOXL2 expression, as compared with other subtypes. Using ChIP-seq to map its genome-wide localization, we found that $\mathrm{H} 3 \mathrm{~K} 4 \mathrm{ox}$ was enriched in heterochromatin in TNBC cells, which are highly metastatic and resistant to chemotherapy. Decreasing LOXL2 levels reduced the amount of H3K4ox in chromatin, resulting in chromatin decondensation and a sustained activation of DDR. Further, both LOXL2 depletion and treatment of TNBC with chromatin-modifying drugs sensitized cancer cells to conventional treatments. Thus, targeting H3K4ox levels may open a new therapeutic window for this subtype of breast cancer.

\section{Results}

\section{Generating an H3K40x-specific antibody}

We initially generated a specific antibody for the recently discovered histone modification of $\mathrm{H} 3 \mathrm{~K} 4 \mathrm{ox}$, as a prerequisite for studying its physiological function. As the aldehyde group generated by LOXL2 reaction on trimethylated lysine 4 is highly reactive, and hence unfit for immunochemical studies, we hypothesized that a primary alcohol might provide a similar oxygen-bearing functionality that is less reactive, in order to generate a modificationspecific antibody that provides a readout of $\mathrm{H} 3 \mathrm{~K} 4 \mathrm{ox}$ (Fig. 1a). We therefore synthesized a $\mathrm{H} 3$ peptide containing a 6-hydroxynorleucine residue as allysine at position 4 and used this for rabbit immunization (Fig. 1b). The resulting H3K4ox antibody was highly specific for the H3K4ox peptide, with very low cross-reactivity for unmodified $\mathrm{H} 3$ and no detected cross-reactivity for H3K9me3 or $\mathrm{H} 3 \mathrm{~K} 4 \mathrm{me} 3$, in a wide range of experimental conditions (dot blots, western blots, and chromatin immunoprecipitation (ChIP) experiments) (Fig. 1c-f). Analysis of purified nucleosomes from $293 \mathrm{~T}$ cells showed that H3K4ox levels increased, and $\mathrm{H} 3 \mathrm{~K} 4 \mathrm{me} 3$ levels decreased, when nucleosomes were incubated with wild-type (wt) recombinant LOXL2 but not with a catalytically inactive LOXL2 (LOXL2mut) [3] (Fig. 1d, upper panel). Moreover, the levels of H3K4ox also increased in MCF-7 cells transfected with LOXL2 as compared with cells transfected with the empty vector (Fig. 1d, lower panel). Finally, MDA-MB-231 cells infected with an shRNA targeting the human LOXL2 (LOXL2 knockdown (KD)) showed a specific reduction in H3K4ox levels as compared with cells infected with an irrelevant shRNA (control), in both western blots and ChIP-PCR experiments using the E-cadherin gene promoter $(C D H 1)$, which is a well-known LOXL2-mediated H3K4 oxidation target promoter (Fig. 1e) [3, 4]. Kinetics of the reaction using recombinant LOXL2 and nucleosomes revealed that levels of intermediate alcohol were maintained for $2 \mathrm{~h}$, after which they were reduced (Fig. 1f). Biotinylated hydrazide was used to detect the generated aldehyde group, which appears after $4 \mathrm{~h}$ of reaction (Fig. 1f). Thus, as the intermediate alcohol is relatively stable, the antibody we generated can be used as a readout of the oxidized histone $\mathrm{H} 3 \mathrm{~K} 4$, although we cannot distinguish whether it recognizes the intermediate alcohol, the aldehyde group, or both.

\section{H3K40x maps to heterochromatin and controls chromatin accessibility in TNBC cells}

As aberrant expression and activity of LOXL2 have been reported in various cancer types [7-9], we checked the levels of LOXL2 and H3K4ox in several breast cancer cell lines representing different subtypes: luminal $\mathrm{A}$, in the $\mathrm{T}-47 \mathrm{D}$ and MCF-7 cell lines $\left(\mathrm{ER}^{+} / \mathrm{HER} 2^{-} / \mathrm{PR}^{+/-}\right)$; luminal $\mathrm{B}$, in the $\mathrm{BT}$ 474 cell line $\left(\mathrm{ER}^{+} / \mathrm{HER}^{+} / \mathrm{PR}^{+/-}\right)$; and basal $\mathrm{TNBC}$, in the human MDA-MB-231 (ER $\left.{ }^{-} / \mathrm{HER}^{-} / \mathrm{PR}^{-}\right)$cell line [25]. As compared with the other cell lines, MDA-MB-231 (TNBC) showed high levels of LOXL2 and a corresponding 
A

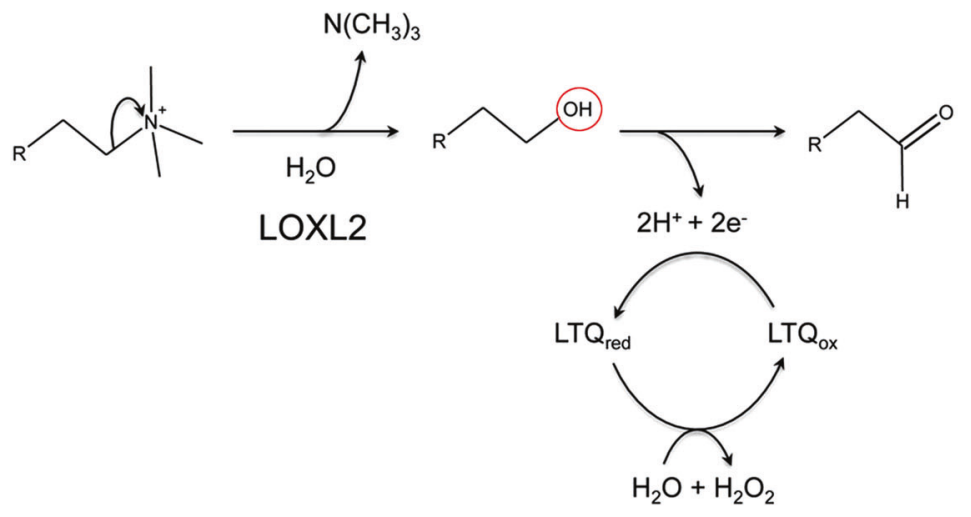

B
C

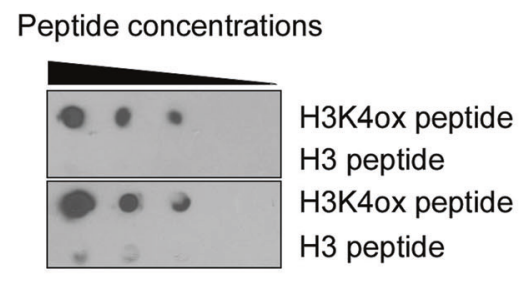

Peptide concentrations

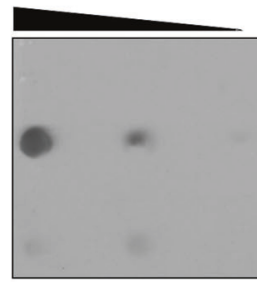

H3K9me3 peptide

H3K40x peptide

H3K4me3 peptide

H3 peptide 6-hydroxynorleucine<smiles>N[C@@H](CCCCc1ccccc1)C(=O)O</smiles>

D LOXL2r wt mut

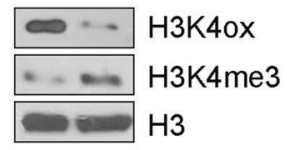

LOXL2 ø +

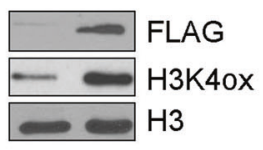

$\mathrm{F}$

E

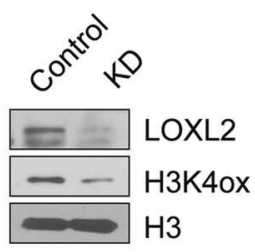

$\mathrm{CDH} 1$

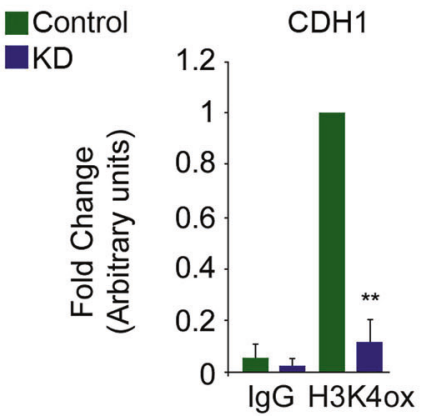

Fig. 1 Quality control of the anti-H3K4ox antibody. a Schematic representation of the LOXL2 reaction. The red circle indicates the intermediate residue that is targeted by the in-house generated antiH3K4ox antibody. b The artificial amino acid 6-hydroxynorleucine was used in the peptide to generate the anti-H3K4ox antibody. c The anti-H3K4ox antibody was found to be specific in western blot in two replicates of dot blots of dilution series of oxidized histone $\mathrm{H} 3$ peptide (H3K4ox) or unmodified H3 peptide (left panel), as well as in a representative dot blot of a dilution series of H3K9me3, H3K4ox, $\mathrm{H} 3 \mathrm{~K} 4 \mathrm{me} 3$, or $\mathrm{H} 3$ peptides (right panel). d Nucleosomes were incubated with recombinant wild-type (wt) LOXL2 or a catalytically inactive LOXL2 (mut) purified from baculovirus to detect H3K4ox/ H3K4me3 levels (upper panel). Lysates of MCF-7 cells transfected

enrichment of H3K4ox (Fig. 2a). Levels of H3K4ox also paralleled increases in LOXL2 expression levels in other TNBC cell lines (e.g., MDA-MB-468, CAL-51, HS-578-T, and BT-549), although with variable LOXL2 expression levels (Fig. 2b). Finally, comparing PDXs from luminal (3 PDXs) or TNBC (6 PDXs) subtypes of breast cancer, we with an empty vector $(\varnothing)$ or with $L O X L 2$ were analyzed by western blotting, using the indicated antibodies (lower panel). e Western blot for LOXL2, H3K4ox, and total H3 from MDA-MB-231 cells infected with short hairpin RNA (shRNA) as a control, or a knockdown (KD) using a shRNA specific for LOXL2 (LOXL2 KD) (left panel). AntiH3K4ox ChIP-PCR was used to analyze the E-cadherin gene ( $\mathrm{CDH1})$ promoter in MDA-MB-231 cells infected with shRNA for either control (green bar) or LOXL2 KD (blue bar). Data of qPCR amplifications were normalized to the input and to total $\mathrm{H} 3$ for each condition. Error bars indicate the SD from at least three independent experiments. $* * P<0.01$. f Western blot of $\mathrm{H} 3 \mathrm{~K} 4 \mathrm{ox}$ and biotin incorporation (BTH-H3) in nucleosomes incubated with recombinant LOXL2 purified from baculovirus, after different incubation times

found that in all TNBC PDXs the levels of H3K4ox were higher compared with luminal PDXs. In addition, in four out of six TNBC PDXs H3K4ox levels also correlated with high LOXL2 expression. (Fig. 2c).

To elucidate the function of H3K4ox in breast cancer cells, we first performed a ChIP-seq experiment with the 
anti-H3K4ox antibody to determine the genomic distribution of H3K4ox in the MDA-MB-231 cells. Peaks called using model-based analysis for ChIP-seq (MACS) [26] showed low differences in H3K4ox between two sequencing replicates, with a genome-wide Pearson correlation coefficient of the read count of the two replicates of 0.997 (Fig. 2d, upper panel). We observed that H3K4ox peaks were distributed throughout different genomic elements (Fig. 2d, lower panel). Using the ChromHMM tool [27], we assessed the statistical overrepresentation of the $\mathrm{H} 3 \mathrm{~K} 4 \mathrm{ox}$ peaks through different chromatin states (promoter, weak or strong enhancer, insulator, repressed, or heterochromatin [nonrepetitive sequences]) and found that $\mathrm{H} 3 \mathrm{~K} 4 \mathrm{ox}$ peaks were significantly overrepresented in heterochromatin (Fig. 2e, f). As generating an aldehyde in $\mathrm{H} 3$ removes a positive charge and creates a very reactive group, we hypothesized that this reaction affects chromatin structure. To test this, we used the assay for transposase-accessible chromatin [28, 29] followed by deep sequencing (ATAC-seq), which exploits the ability of the prokaryotic transposase $\mathrm{Tn} 5$ to integrate preferentially into accessible (open) chromatin. ATAC-seq showed an increased ATAC signal in LOXL2 KD cells, but not with control cells, at H3K4ox-positive sites (Fig. 2g). These results were validated in selected regions by ChIPqPCR and ATAC-qPCR in control and LOXL2 KD cells: H3K4ox enrichment decreased in LOXL2 KD cells, with a correlating increase of ATAC signal, in these regions (Fig. 2h). No changes were observed in a control promoter, POL2RA (Fig. 2h). These data demonstrated that, in the absence of LOXL2, H3K4ox levels decrease and chromatin adopts a more open conformation (Fig. 2g). Thus, our results showed that $\mathrm{H} 3 \mathrm{~K} 4 \mathrm{ox}$ is enriched in heterochromatin and is directly linked with chromatin accessibility in those regions.

\section{Chromatin structure alterations activate DDR in a LOXL2-dependent manner}

As the chromatin state can influence many aspects of DDR [30], we hypothesized that disruption of LOXL2 expression and impairment of $\mathrm{H} 3 \mathrm{~K} 4 \mathrm{ox}$ generation might influence DDR by affecting chromatin accessibility. To test this, we analyzed by immunofluorescence MDA-MB-231 cells that had been infected with either LOXL2 KD or control lentiviruses, using two well-established markers of DDR: phosphorylated $\mathrm{H} 2 \mathrm{AX}(\gamma-\mathrm{H} 2 \mathrm{AX})$ and TP53-binding protein 1 (53BP1). Depletion of LOXL2 (using LOXL2 KD) led to more foci of both $\gamma-\mathrm{H} 2 \mathrm{AX}$ and 53BP1 than in control cells, suggesting that LOXL2 KD cells may accumulate DNA breaks and/or activate DDR (Fig. 3a). To determine if the LOXL2 catalytic activity was involved in the observed phenotype, LOXL2 KD cells were complemented by reinfection with ectopic vector expressing either the wt LOXL2IRES-GFP or a catalytically inactive LOXL2 (LOXL2mutIRES-GFP), both of which were expressed at similar levels (Fig. S1). Fewer $\gamma-\mathrm{H} 2 \mathrm{AX}$ and 53BP1 foci were observed in LOXL2 KD cells after reintroduction of the wt (but not of the catalytically inactive) LOXL2 (Fig. 3b), establishing that suppressing DDR activation requires both the activity of LOXL2 and H3K4ox generation.

\section{LOXL2 KD activates DDR independently of DNA damage}

Increased DDR activation in LOXL2-depleted cells could be a consequence of more DSBs due to reduced H3K4ox levels and/or chromatin decondensation. Notably, aberrant silencing and conflicts between replication forks and transcription, as well as the presence of R-loops (a transcriptional intermediate), can result in DNA damage and are influenced by chromatin state, for example in cells lacking the linker histone H1 [31]. However, our analysis of RNAseq data revealed that $L O X L 2 \mathrm{KD}$ cells did not have altered expression of repetitive elements (Table 1), and DDR activation in LOXL2 KD cells was not affected by cordycepin, an inhibitor of RNA synthesis that abolishes R-loop formation [32, 33] (Fig. 3c). As R-loops can generate DNA damage during replication due to fork stalling and collapse $[32,34]$, we next analyzed $\gamma-\mathrm{H} 2 \mathrm{AX}$ and 53BP1 foci in noncycling $L O X L 2 \mathrm{KD}$ cells and found DDR activation to also be increased (Fig. 3d). Overall, these data suggested that overexpression of repetitive elements, R-loop formation, and replication fork stalling were not responsible for activating DDR following LOXL2 depletion, when heterochromatin adopts a more open state. As chromatin structure alterations can trigger the DDR, even in the absence of DNA damage [22, 35, 36], we checked for the presence of DNA damage following LOXL2 depletion more directly, using the comet assay, in LOXL2 KD or control cells under alkaline conditions. No increases in DNA damage (due to either single-strand or double-strand DNA breaks) were observed in LOXL2 KD cells (Fig. 3e). Moreover, we did not observe any significant differences in chromosomal lesions between LOXL2 KD or control cells in metaphase spreads (Fig. 3f). Finally, analyzing for mitotic aberrations (anaphase bridges and micronuclei), which can be indicative of replication stress or DNA repair defects, we observed only a mild increase in anaphase bridges in the absence of LOXL2 (Fig. S2). Taken together, our data suggested that the combination of loss of LOXL2 and reduced $\mathrm{H} 3 \mathrm{~K} 4 \mathrm{ox}$ levels in TNBC cells was sufficient to activate the DDR in the absence of detectable DNA lesions. 


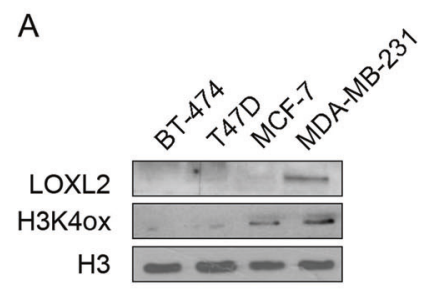

D
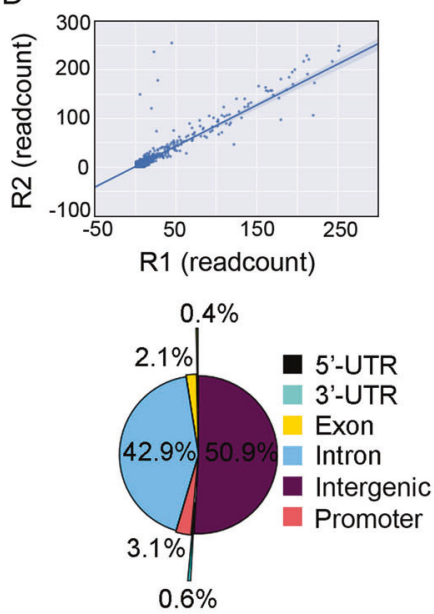

G

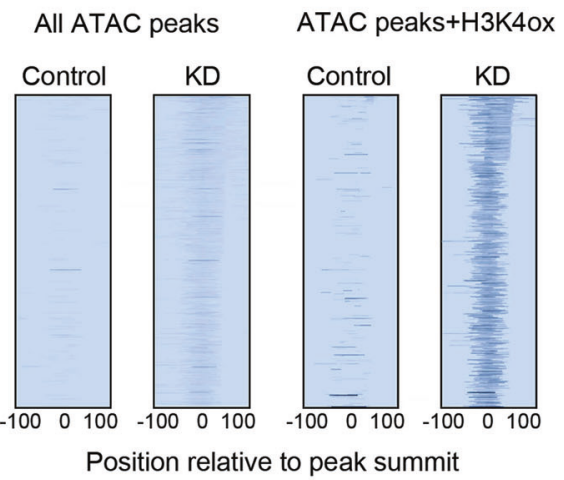

\section{Alterations in chromatin compaction activate the DDR}

To further address the origin of DDR signaling in LOXL2depleted cells, we analyzed the behavior of additional DDR signaling components. As both $\gamma$-H2AX and 53BP1 foci formation require the ATM kinase in some settings, we
C

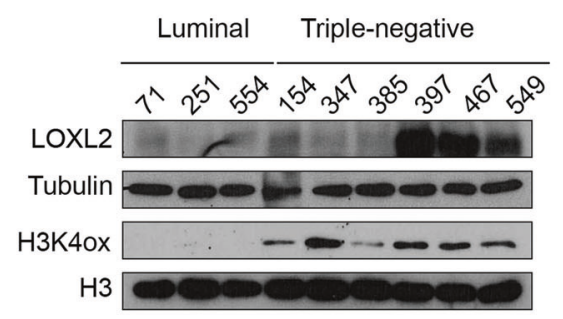

$\mathrm{F}$
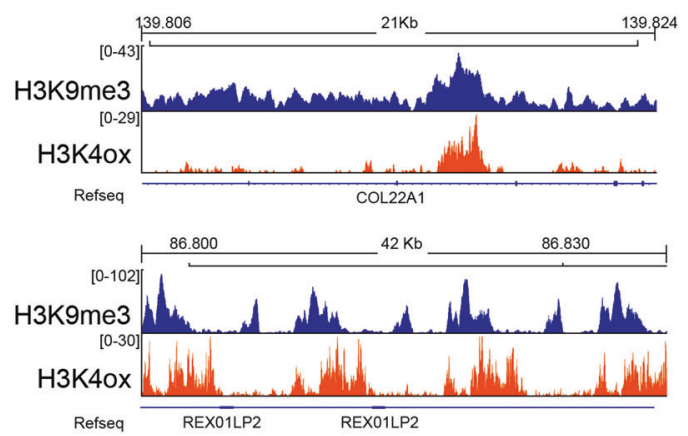

$\mathrm{H} \underset{\mathrm{K}}{\mathrm{C} \text { Control }}$

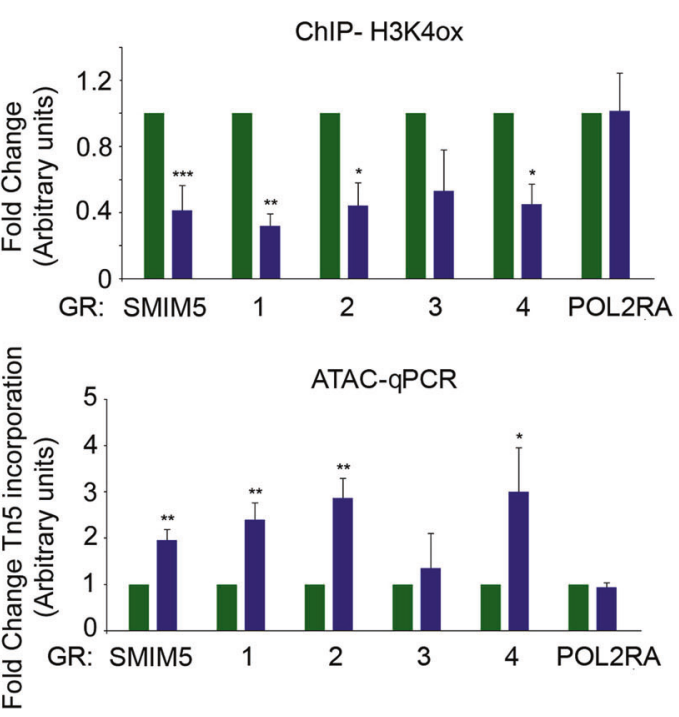

treated LOXL2 KD and control cells with the ATM inhibitor KU55933 and analyzed foci formation (Fig. 4a). Decreased foci of both markers were observed upon ATM inhibition, indicating that LOXL2-induced DDR was largely ATMdependent. Consistent with this, LOXL2 KD cells had increased phosphorylation of several ATM substrates, including KAP-1, CHK1, and CHK2 (Fig. 4b). However, 
Fig. $2 \mathrm{H} 3 \mathrm{~K} 4 \mathrm{ox}$ maps to heterochromatin and controls chromatin accessibility in TNBC cells. Western blot for the indicated antibodies in a panel of breast cancer cell lines (a), TNBC cell lines (b), or PDXs (c). d Pearson correlation between two H3K4ox sequencing replicates. Distribution of all H3K4ox ChIP-seq peaks in MDA-MB-231 cells are given, with the indicated percentages. e Contingency table of the Fisher's exact test showed the statistical overrepresentation of the H3K4ox peaks through different chromatin states. f Genome browser view of $\mathrm{H} 3 \mathrm{~K} 4 \mathrm{ox}$ and $\mathrm{H} 3 \mathrm{~K} 9 \mathrm{me} 3$-binding profiles at two representative heterochromatin regions. g Heatmaps show the ATAC signal in all peaks as well as in peaks that overlap with $\mathrm{H} 3 \mathrm{~K} 4 \mathrm{ox}$ in LOXL2 KD or control cells. h H3K4ox ChIP-PCR validation of the selected genomic regions from the ChIP-seq from control or LOXL2 KD MDA-MB-231 cells. Data of qPCR amplification were normalized to the input and to total H3 (upper panel). ATAC-qPCR validation of the incorporation of the transposase Tn5 at the selected genomic regions from the ChIP-seq from control or LOXL2 KD MDA-MB-231 cells. Data of qPCR amplification were normalized to an unchanging genomic region (the HPRT promoter) and expressed as the fold-change relative to data obtained from control cells, which were set to 1 (lower panel). In both experiments, the RNA polymerase II (POL2RA) promoter was used as a negative control. Error bars indicate the SD from at least three independent experiments. ${ }^{*} P<0.05, * * P<0.01$, $* * * P<0.001$

we ruled out that increased DDR signaling was due to apoptosis in LOXL2 KD cells, as no cleaved caspase-3 signal was observed in either LOXL2 KD or control cells (Fig. S3a). As these data suggested that the LOXL2 KD cells activated a checkpoint response, we analyzed cell cycle progression following LOXL2 depletion. After synchronization with a double thymidine block, LOXL2 KD cells were not able to efficiently progress through the cell cycle (Fig. 4c), and western blotting for H3S10-P showed that this histone mark was undetectable in LOXL2 KD cells as compared with control cells. (Fig. 4d). These data strongly suggested that LOXL2 KD cells arrested primarily in G1. Consistent with this possibility, cell proliferation capacity of LOXL2 KD cells was blocked (Fig. 4e, upper panel), and their colony-formation capacity was strongly reduced after only a few passages (Fig. 4e, lower panel). This effect on proliferation was further confirm by gene ontology analysis of the gene expression pattern of LOXL2 KD cells (Fig. S3b; Tables 2 and 3).

To test whether the effects on chromatin in LOXL2 KD cells directly activated ATM-dependent DDR signaling, we forced chromatin condensation in these cells by expressing the linker histone $\mathrm{H} 1$ or the H3K9 methyltransferase SUV39H1 (as green fluorescent protein (GFP)-labeled proteins) and counted the number of $\gamma-\mathrm{H} 2 \mathrm{AX}$ foci in GFP-positive cells. Notably, overexpression of either $\mathrm{H} 1^{\mathrm{GFP}}$ or SUV$39^{\mathrm{GFP}}$ in $L O X L 2 \mathrm{KD}$ cells reduced the number of $\gamma-\mathrm{H} 2 \mathrm{AX}$ foci as compared with LOXL2 KD cells that expressed GFP alone $\left(+\right.$ MOCK $^{\mathrm{GFP}}$ ) (Fig. 4f, g). This suggested that lack of LOXL2 and reduced $\mathrm{H} 3 \mathrm{~K} 4 \mathrm{ox}$ levels affected the regulation of chromatin condensation (leading to decondensation) and activated DDR, even in the absence of DNA damage.

\section{LOXL2 reduction enhances chemosensitivity of TNBC cells}

We found that reducing H3K4ox levels via LOXL2 depletion led to chromatin decondensation, triggered DDR activation, and induced cell cycle arrest, suggesting that LOXL2 inhibition could be interesting as a breast cancer treatment. As no LOXL2-specific inhibitors are currently available, we therefore tested whether reducing the levels of functional LOXL2 would increase the sensitivity of breast cancer cells to chemotherapy. For this, we treated three different TNBC cell lines with doxorubicin (a topoisomerase inhibitor that generates DSBs) either alone or together with trichostatin A (TSA) (a general HDCA inhibitor [37] that increases chromatin accessibility [38]). Indeed, we observed that in all cases, treatment of doxorubicin together with TSA increased the percentage of cell death-indicative of an increased cell sensitivity to chemotherapy (Fig. 5a). Similar results were obtained with two TNBC_PDXs (PDX549 and PDX-154) (Fig. 5a). To determine if these results were reproducible in vivo, PDX-549 cells were first grown ex vivo, and then $10^{6}$ cells were subcutaneously implanted in nude mice. After tumor formation, mice were treated with TSA, doxorubicin, doxorubicin plus TSA, or (as a control) vehicle for 25 days. In agreement with our previous results, we observed that tumor growth was substantially impaired with the combined TSA/doxorubicin treatment (Fig. 5b).

\section{Discussion}

In this work, we found that several TNBC cell lines and PDXs express high levels of LOXL2 as compared with those of the luminal breast cancer subtype. With the use of a newly generated antibody, we were able to detect the H3K4ox modification produced by LOXL2 [3] and we observed how this histone $\mathrm{H} 3$ modification was higher in all TNBC cell lines and PDXs as compared with luminal subtypes. This H3 modification is enriched in heterochromatin and is required to maintain the condensed heterochromatin state. It is possible that during tumor progression, some cancer cells undergo the EMT program and start to express LOXL2. The transcription factor Snail1, together with LOXL2, would participate in downregulating the $C D H 1$ gene and other heterochromatin transcripts, giving rise to transformation of cancer epithelial cells into mesenchymal cells (through EMT) [6, 39-41]. Recently, two different groups have suggested that EMT is dispensable for lung and pancreas metastasis but contributes significantly to the formation of recurrent metastasis after chemotherapy [42, 43]. This finding is in agreement with our results, in which we observed that the most aggressive and resistant subtype of breast cancer (TNBC) has a 

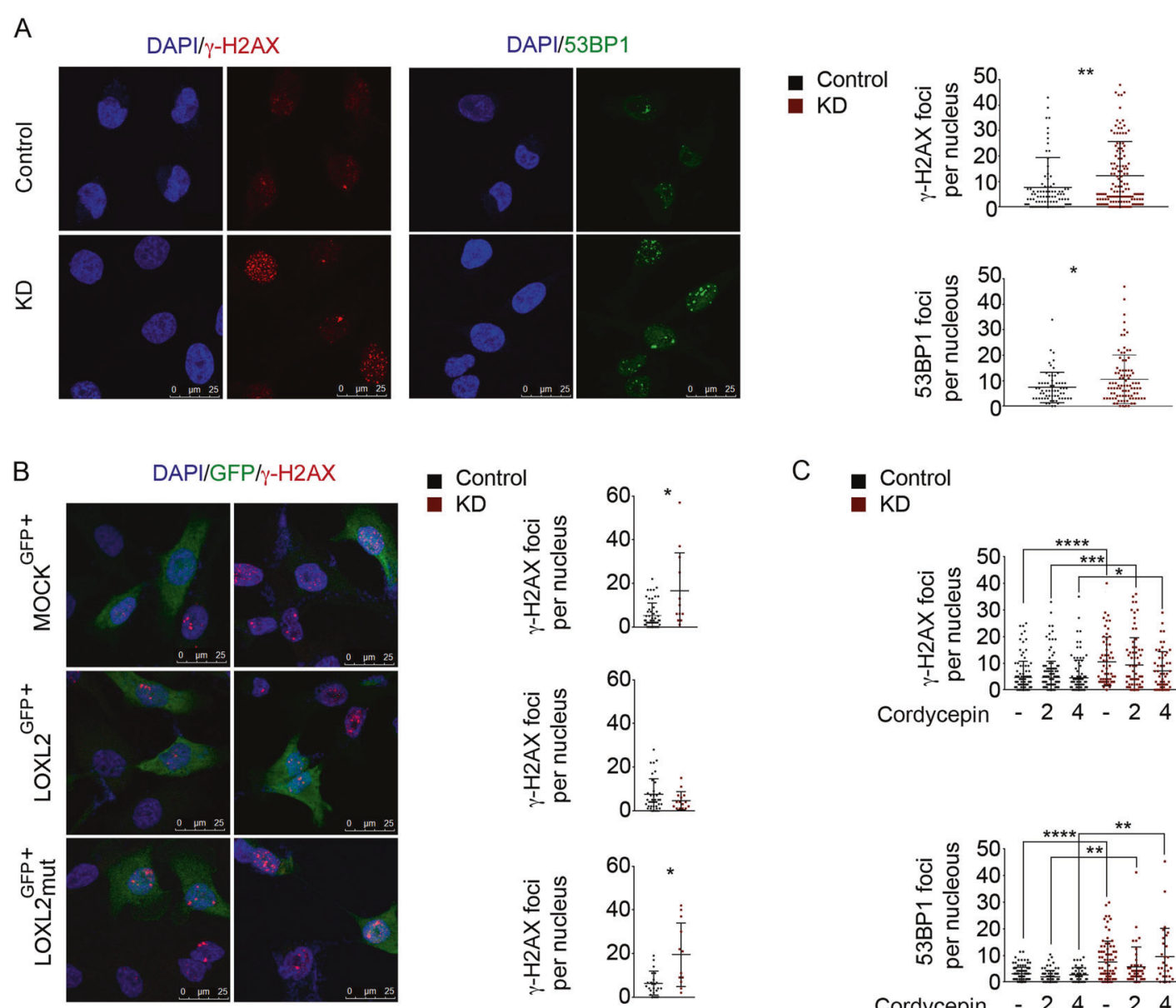

C Control
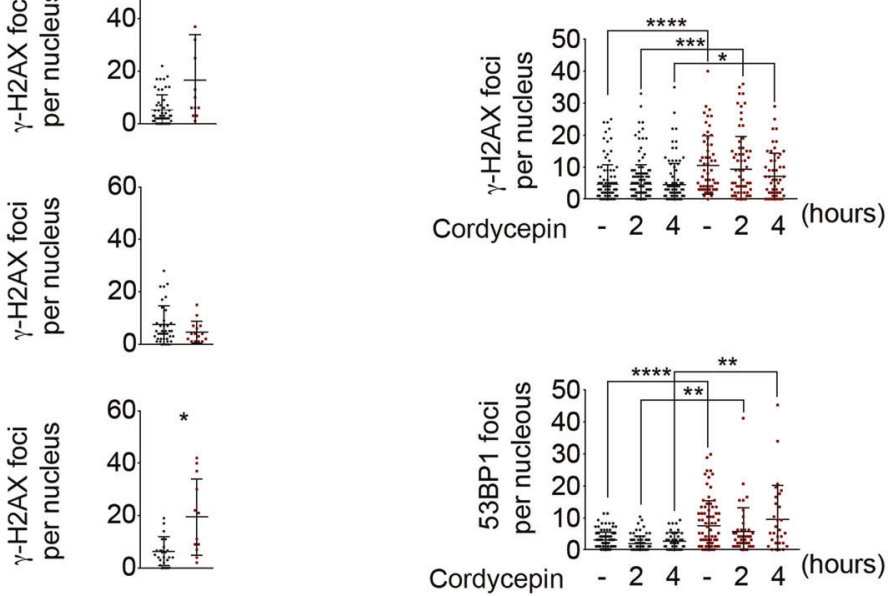

D

$\mathrm{E}$

DAPI/53BP $1 / \gamma-\mathrm{H} 2 \mathrm{AX}$
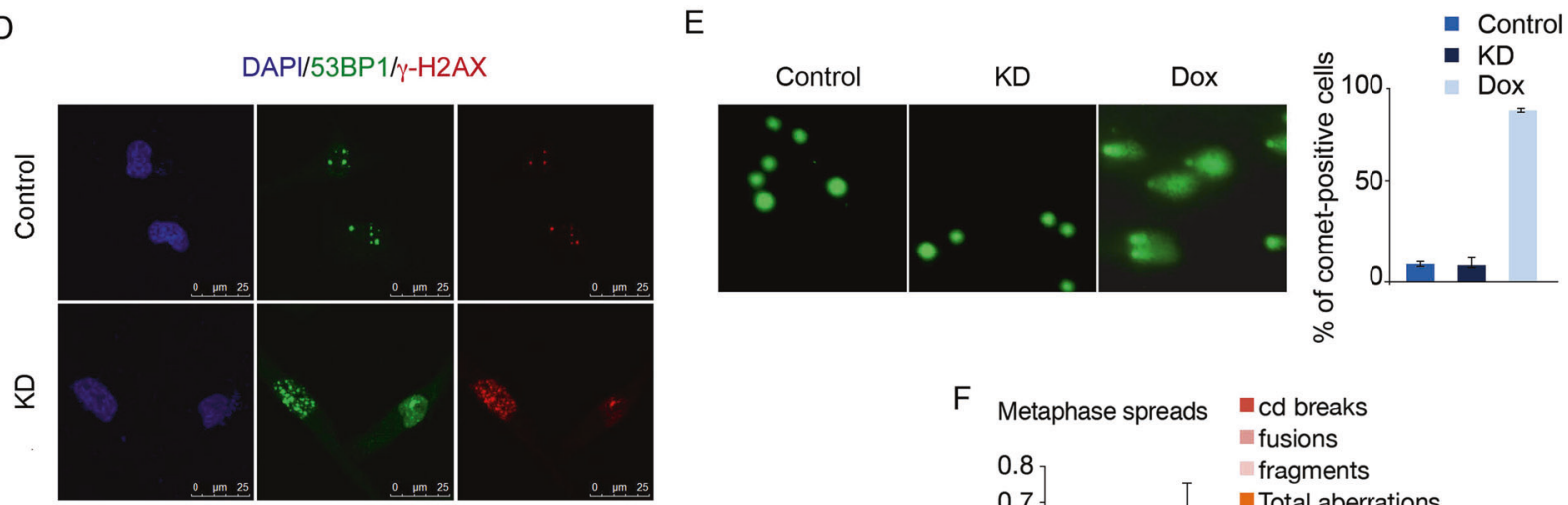

- Control

- KD
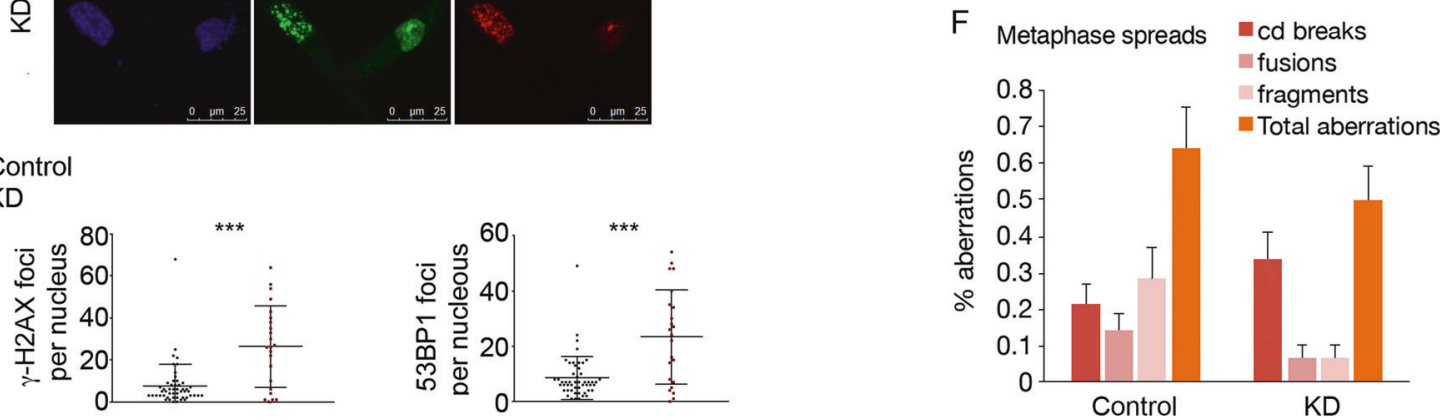
Fig. 3 Chromatin opening activates DDR via a mechanism that requires catalytically functional LOXL2 but can be independent of DNA damage. a $\gamma-\mathrm{H} 2 \mathrm{AX}$ and 53BP1 staining and foci quantification are shown by immunofluorescence with a specific antibody for $\gamma$-H2AX (left image) or for 53BP1 (right image). Dot graphs indicate the number of foci for $\gamma$ $\mathrm{H} 2 \mathrm{AX}$ (upper graph) and 53BP1 (lower graph) per cell in control and LOXL2 KD conditions. b $\gamma$-H2AX staining and foci quantification are shown by immunofluorescence with the indicated antibody after LOXL2 reinfection. MDA-MB-231 cells were first infected with control or LOXL2 KD lentivirus and then, after puromycin selection, again with GFP $\left(\mathrm{MOCK}^{\mathrm{GFP}+}\right.$ ), wild-type LOXL2-IRES-GFP (LOXL2 ${ }^{\mathrm{GFP}+}$ ), or LOXL2mut-IRES-GFP (LOXL2mut ${ }^{\mathrm{GFP}+}$ ). Cells were fixed after $24 \mathrm{~h}$. Dot graphs indicate the number of $\gamma$-H2AX foci per GFP-positive cells containing $\mathrm{MOCK}^{\mathrm{GFP}+}$ (upper graph), LOXL2 $2^{\mathrm{GFP}+}$ (middle graph), or LOXL2mut ${ }^{\mathrm{GFP}+}$ (lower graph). $\mathrm{c}$ Dot graphs indicate the number of $\gamma$ $\mathrm{H} 2 \mathrm{AX}$ (upper graph) and 53BP1 (lower graph) foci per cell in control and LOXL2 KD cells after treatment with $200 \mu \mathrm{M}$ cordycepin for the indicated timepoints. d $\gamma-\mathrm{H} 2 \mathrm{AX}$ and 53BP1 staining and foci quantification are shown after immunofluorescence with the indicated antibodies in non-replicative conditions. Dot graphs indicate the number of the $\gamma-\mathrm{H} 2 \mathrm{AX}$ (left graph) and 53BP1 (right graph) foci in control and LOXL2 KD cells. e Representative image showing DNA damage in control or LOXL2 KD MDA-MB-231 cells, visualized by the alkaline comet assay. Cells were treated with $0.3 \mu \mathrm{M}$ doxorubicin for $24 \mathrm{~h}$. The graph shows the percentage of MDA-MB-231 positive cells. f Chromosome alterations in control and LOXL2 KD MDA-MB231 cells. Error bars indicate the SD from at least three independent experiments. $* P<0.05$, $* * P<0.01$, and $* * * P<0.001$, $* * * * P<$ 0.0001

mesenchymal phenotype and is enriched for $\mathrm{H} 3 \mathrm{~K} 4 \mathrm{ox}$ in its heterochromatin. We observed that reducing H3K4ox levels perturbed the balance between condensed and decondensed chromatin and activated the DDR. Similar phenotypes have been previously observed after depletion of SUV-39, PRDM2, or HP1, which are required for heterochromatin maintenance and cell survival after DNA damage [23, 24, 44, 45]. H3K4ox levels have been correlated to the repression of $\mathrm{CDH} 1$ and major satellites (heterochromatin transcripts) during the EMT program [3, 4, 6]. CDH1 is upregulated in TNBC cells when LOXL2 is knocked down and the levels of H3K4ox are decreased; however, the H3K4ox modification does not seem to be involved in repression of heterochromatin transcripts, as we did not observe increased levels of heterochromatin transcripts in LOXL2 KD conditions. Notably, downregulation of heterochromatin transcripts during EMT occur at a specific timepoint during chromatin reorganization, after which the levels of transcription recovered despite the presence of H3K4ox. Intriguingly, although upstream DDR signaling is sustained when chromatin is maintained in a condensed state [22, 46, 47], we found that forcing chromatin decondensation was sufficient to activate DDR signaling in the absence of any detectable DNA lesions. It seems that an abnormal, unbalanced ratio between condensation and decondensation leads to persistent DDR signaling. As LOXL2 has multiple substrates besides histones [5], we cannot discard an additional putative role for LOXL2 in the direct regulation of the DDR machinery. However, we observed that the DDR induced in the absence of LOXL2 can be inhibited by forcing chromatin to condense using either of two different approaches, suggesting that the main molecular mechanism is the regulation per se of the chromatin compaction state.

While a correlation between chromatin compaction and DDR has been previously reported [22, 46, 47], we demonstrated here that $\mathrm{H} 3$ oxidation by LOXL2 is another molecular mechanism that maintains compaction. Notably, LOXL2 has been widely linked to cancer and the acquisition of cellular malignancy, as it is overexpressed in many tumors [15, 48-51] and has an important role in tumor formation [52].

Induction of chromatin compaction has been suggested as a potential therapeutic tool in gliomas, in which chromatin compaction limits DDR [46] and promotes damage resistance [53]. However, this does not appear to apply to TNBC cells. In support of this, we have now shown that several TNBC PDX tumors treated with TSA, a histone deacetylase inhibitor that generates an open chromatin state, enhanced tumor killing with doxorubicin in these cells. These observations suggest a rationale for studying combinations of "chromatin opening drugs," including inhibitors of histones deacetylases, EZH2, or LSD1, as a strategy for overcoming resistance to treatment in TNBC.

\section{Materials and methods}

\section{Cell lines, transfections, and infections}

Most of the cell lines used in this work (the TNBC lines MDA-MB-468 (ATCC HTB-132), CAL-51 (CSC-C0382), HS-578T (ATCC HTB-126), and MDA-MB-231 (ATCC HTB-26), the luminal A T-47D (ATCC HTB-133), MCF-7 (ATCC HTB-22D), and luminal B BT-474 (ATCC HTB20)) as well as cells isolated from PDXs, were maintained in Dulbecco's modified Eagle's medium (Biowest; L0106500); the TNBC line BT-549 (ATCC HTB 122) was maintained in Roswell Park Memorial Institute medium (Biowest; L0501-500). All cell cultures were supplemented with 10\% fetal bovine serum (Gibco; 10270106), 2 mM Lglutamine (Biowest; X0550-100), and $1 \%$ penicillinstreptomycin (Gibco; 15140122) at $37^{\circ} \mathrm{C}$ in $5 \% \mathrm{CO}_{2}$. All cell lines were regularly tested for the absence of mycoplasma using standard polymerase chain reaction (PCR). For LOXL2-Flag overexpression assays, MCF-7 cells were seeded for $24 \mathrm{~h}$ and transfected with $10 \mu \mathrm{g}$ pcDNA3hLOXL2-Flag vector using polyethylenimine polymer (Polysciences Inc; 23966-1). Lentiviral particles were produced in HEK293T cells (ATCC CRL-3216) for lentiviral infections to produce LOXL2 KD cells. Cells were grown to 
Table 1 Differential expression analysis between control and LOXL2 knockdown of repetitive elements

\begin{tabular}{|c|c|c|c|c|}
\hline Locus & Ctrl_mean & Lox12_mean & Prob & $\log 2 \mathrm{FC}$ \\
\hline Alu & $3,601,718.96$ & $3,714,313.36$ & 1.00 & 0.04 \\
\hline RNA & $2,918,334.55$ & $3,045,475.41$ & 0.99 & 0.06 \\
\hline L1 & $2,837,069.25$ & $2,730,921.52$ & 0.97 & -0.06 \\
\hline ERV1 & $370,405.10$ & $353,869.12$ & 0.95 & -0.07 \\
\hline TcMar-Tigger & $239,662.63$ & $252,421.97$ & 0.87 & 0.07 \\
\hline UCON19 & 0.51 & 1.23 & 0.76 & 1.28 \\
\hline UCON18 & 0.51 & 1.23 & 0.76 & 1.28 \\
\hline hAT-Tip100 & $34,122.72$ & $38,990.93$ & 0.76 & 0.19 \\
\hline ERVK & $8,958,641.10$ & $9,448,789.20$ & 0.76 & 0.08 \\
\hline MIR & $673,530.34$ & $692,895.61$ & 0.76 & 0.04 \\
\hline hAT & $12,908.30$ & $16,757.15$ & 0.76 & 0.38 \\
\hline TcMar-Mariner & $33,897.47$ & $38,475.09$ & 0.75 & 0.18 \\
\hline UCON4 & 234.62 & 360.51 & 0.74 & 0.62 \\
\hline hAT-Charlie & $374,265.61$ & $380,188.93$ & 0.72 & 0.02 \\
\hline UCON16 & 6.58 & 10.85 & 0.72 & 0.72 \\
\hline Penelope & 7.08 & 11.83 & 0.70 & 0.74 \\
\hline ERVL-MaLR & $399,009.16$ & $406,080.51$ & 0.70 & 0.03 \\
\hline UCON31 & 61.20 & 87.49 & 0.70 & 0.52 \\
\hline MER130 & 15.18 & 21.75 & 0.69 & 0.52 \\
\hline $\mathrm{UCON} 2$ & 320.19 & 434.63 & 0.69 & 0.44 \\
\hline UCON10 & 7.09 & 10.93 & 0.67 & 0.63 \\
\hline UCON28c & 10.12 & 14.81 & 0.63 & 0.55 \\
\hline Eulor4 & 1.26 & 1.98 & 0.55 & 0.65 \\
\hline UCON12 & 14.14 & 4.94 & 0.52 & -1.52 \\
\hline TcMar-Tc2 & $12,524.35$ & $13,658.11$ & 0.51 & 0.13 \\
\hline UCON24 & 1.27 & 0.49 & 0.47 & -1.36 \\
\hline UCON17 & 3.03 & 1.23 & 0.46 & -1.30 \\
\hline ERVL & $174,857.94$ & $177,852.71$ & 0.44 & 0.02 \\
\hline MamRep605 & 7848.87 & 8610.92 & 0.41 & 0.13 \\
\hline UCON28a & 201.88 & 253.61 & 0.39 & 0.33 \\
\hline Satellite & $28,748.18$ & $25,760.18$ & 0.37 & -0.16 \\
\hline UCON26 & 532.62 & 618.38 & 0.35 & 0.22 \\
\hline SVA_B & 2696.77 & 3025.33 & 0.31 & 0.17 \\
\hline acro & 14.16 & 10.88 & 0.30 & -0.38 \\
\hline UCON11 & 6.08 & 3.93 & 0.29 & -0.63 \\
\hline UCON15 & 4.29 & 2.25 & 0.29 & -0.93 \\
\hline MuDR & 1610.89 & 1799.90 & 0.28 & 0.16 \\
\hline SVA_F & 3968.24 & 4322.50 & 0.26 & 0.12 \\
\hline Gypsy & $18,034.32$ & $16,836.16$ & 0.26 & -0.10 \\
\hline DNA & 5850.42 & 6284.72 & 0.25 & 0.10 \\
\hline Dong-R4 & 3696.41 & 3198.15 & 0.25 & -0.21 \\
\hline UCON9 & 18.69 & 12.78 & 0.24 & -0.55 \\
\hline Centre & $11,923.53$ & $12,544.21$ & 0.22 & 0.07 \\
\hline Merlin & 297.42 & 327.87 & 0.19 & 0.14 \\
\hline SVA_D & $11,475.76$ & $11,978.90$ & 0.19 & 0.06 \\
\hline UCON28b & 26.33 & 30.80 & 0.18 & 0.23 \\
\hline
\end{tabular}

Table 1 (continued)

\begin{tabular}{|c|c|c|c|c|}
\hline Locus & Ctrl_mean & Lox12_mean & Prob & $\log 2 \mathrm{FC}$ \\
\hline LTR & 1842.20 & 1987.87 & 0.18 & 0.11 \\
\hline UCON8 & 75.37 & 65.33 & 0.15 & -0.21 \\
\hline TcMar & 8328.59 & 8597.66 & 0.11 & 0.05 \\
\hline SVA_A & 1741.04 & 1827.45 & 0.10 & 0.07 \\
\hline CR1 & $85,288.67$ & $84,328.51$ & 0.09 & -0.02 \\
\hline Helitron & 5017.41 & 5157.97 & 0.06 & 0.04 \\
\hline UCON6 & $1,277.83$ & $1,325.68$ & 0.06 & 0.05 \\
\hline RTE & $30,497.19$ & $29,901.20$ & 0.06 & -0.03 \\
\hline PiggyBac & 4590.52 & 4349.34 & 0.06 & -0.08 \\
\hline UCON22 & 12.14 & 10.47 & 0.06 & -0.21 \\
\hline UCON5 & 11.14 & 9.87 & 0.06 & -0.17 \\
\hline telo & 489.07 & 507.40 & 0.05 & 0.05 \\
\hline L2 & $614,402.54$ & $610,776.39$ & 0.05 & -0.01 \\
\hline Deu & 2157.42 & 2220.57 & 0.05 & 0.04 \\
\hline hAT-Blackjack & $11,106.77$ & $11,287.96$ & 0.05 & 0.02 \\
\hline UCON1 & 3.04 & 3.25 & 0.04 & 0.10 \\
\hline UCON27 & 72.82 & 74.65 & 0.03 & 0.04 \\
\hline UCON20 & 12.13 & 10.95 & 0.02 & -0.15 \\
\hline MamRep564 & 756.04 & 712.80 & 0.02 & -0.08 \\
\hline RTE-BovB & 1064.66 & 1011.37 & 0.02 & -0.07 \\
\hline ERV & 440.91 & 422.61 & 0.01 & -0.06 \\
\hline SINE & 4912.94 & 4894.14 & 0.00 & -0.01 \\
\hline UCON25 & 2.27 & 2.20 & 0.00 & -0.04 \\
\hline SVA_E & 1087.33 & 1076.64 & 0.00 & -0.01 \\
\hline
\end{tabular}

$70 \%$ confluency and then transfected by dropwise addition of $\mathrm{NaCl}(150 \mathrm{mM})$, a DNA mixture of pLKO-short hairpin control (shCT)/shLOXL2 (7.5 $\mu \mathrm{g})$, pCMV-VSVG $(1.5 \mu \mathrm{g})$, pMDLg/pRRE $(4.5 \mu \mathrm{g})$, and pRSV-Rev $(1.5 \mu \mathrm{g})$, and polyethylenimine polymer $(1 \mathrm{mg} / \mathrm{ml})$ (Polysciences Inc), which had been preincubated for $15 \mathrm{~min}$ at room temperature. Transfection medium was replaced with fresh medium after $24 \mathrm{~h}$ (day 1). The supernatant from the transfection medium at days 2 and 3 was filtered with $0.45 \mu \mathrm{m}$ filter unit (Merck Millipore) and stored at $4{ }^{\circ} \mathrm{C}$ (both were mixed after day 2). The supernatant mixture was concentrated using Lenti-X Concentrator product (Clontech) and centrifuged at $1500 \times \mathrm{g}$ for $45 \mathrm{~min}$ at $4{ }^{\circ} \mathrm{C}$. The pellet was resuspended in $1 \mathrm{ml}$ fresh medium, aliquoted $(100 \mu \mathrm{l})$, and stored at $-20^{\circ} \mathrm{C}$. Aliquots were used to infect MDA-MB-231 cells [6]. For retroviral infections, HEK293 gag-pol cells were used to produce retroviral particles. Cells were transfected as for HEK293T cells with a mixture of $\mathrm{NaCl}(150 \mathrm{mM})$, DNA ( $2.5 \mu \mathrm{g}$ of pCMV-VSV-G and $7.5 \mu \mathrm{g}$ of pMSCV, pMSCVLOXL2 wt-FLAG or pMSCV-LOXL2 mutFLAG IRES-GFP vectors) and polyethylenimine polymer (1 mg/ml) (Polysciences Inc; 23966-1) that had been preincubated for $15 \mathrm{~min}$ at room temperature; transfection 


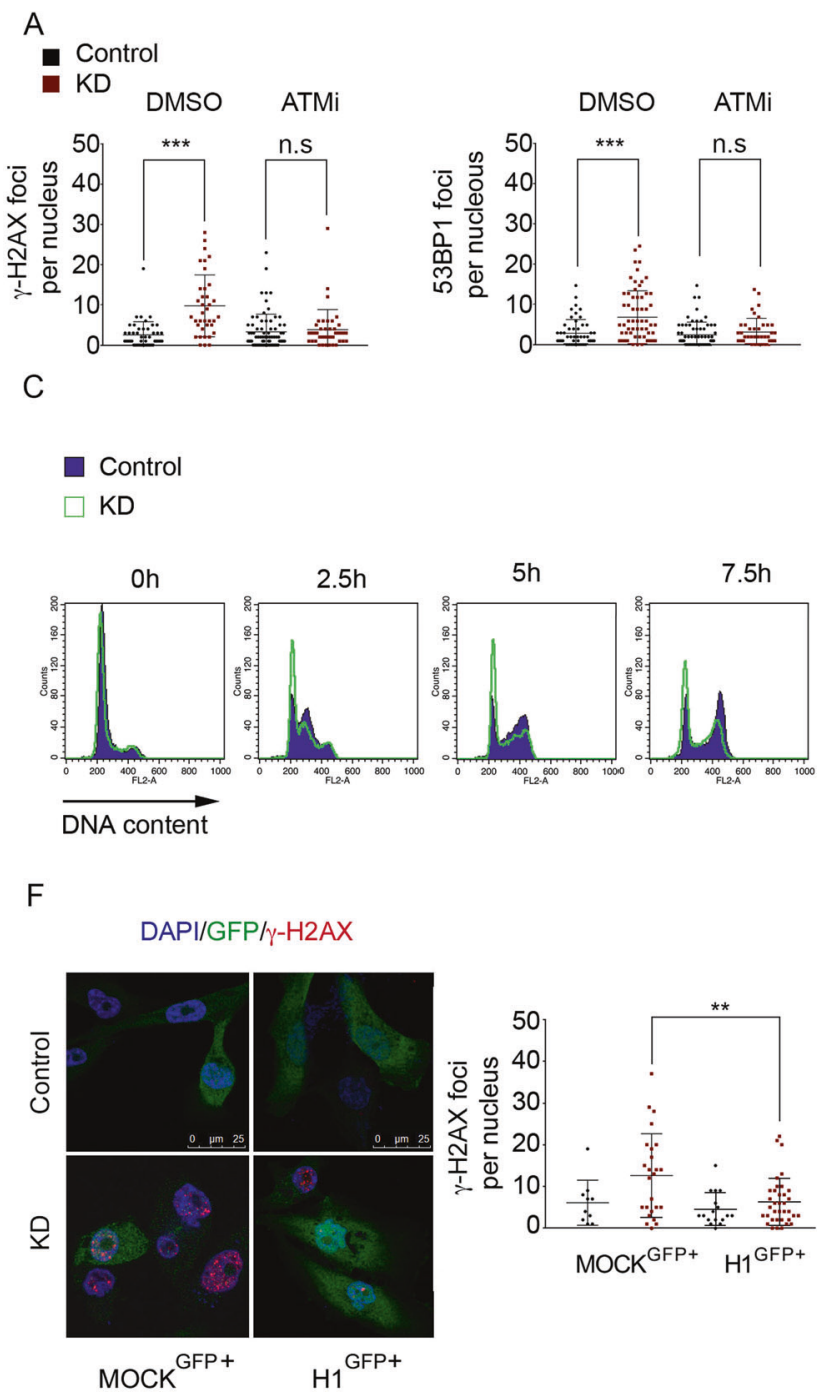

Fig. 4 DDR activation is linked to chromatin decondensation in the absence of LOXL2. a Dot graphs indicate the number of foci with $\gamma$ H2AX (left graph) and 53BP1 (right graph) per cell from control and LOXL2 KD cells treated with DMSO or the ATM inhibitor KU55933. b KAP-1 phosphorylation in control or LOXL2 KD cells was analyzed by western blot. Tubulin and total KAP-1 were used as loading controls. As a positive control, MDA-MB-231 cells treated with $0.1 \mu \mathrm{M}$ doxorubicin for $8 \mathrm{~h}$ (to generate DSBs) were used. Intervening lanes were removed as indicated (left panel). Chk1 and Chk2 phosphorylation in control and LOXL2 KD cells were analyzed by western blot. Chk1 phosphorylation was detected using the anti-P(S317) Chk1 antibody. For phosphorylated Chk2, a shift was detected using an antitotal Chk2 antibody. GADPH was used as a loading control. All samples were obtained under the same experimental conditions; in addition, positive control samples (irradiated fibroblasts) were run on the same gel as their corresponding control and LOXL2 KD samples. Intervening lanes in the Chk1/2 and GADPH blots were removed as

medium was replaced with fresh medium at 24 and $48 \mathrm{~h}$ and processed as explained above. Viral particles were concentrated using Retro-X Concentrator product (Clontech; 631456) and then used to infect MDA-MB-231 cells. HEK293T cells were used to produce lentiviral particles for
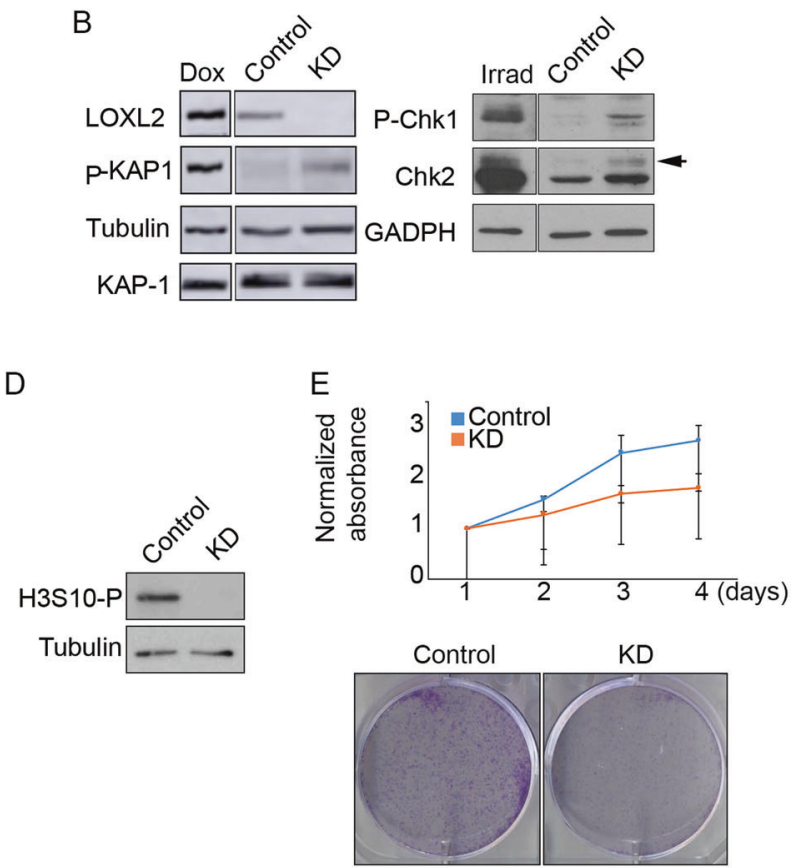

G

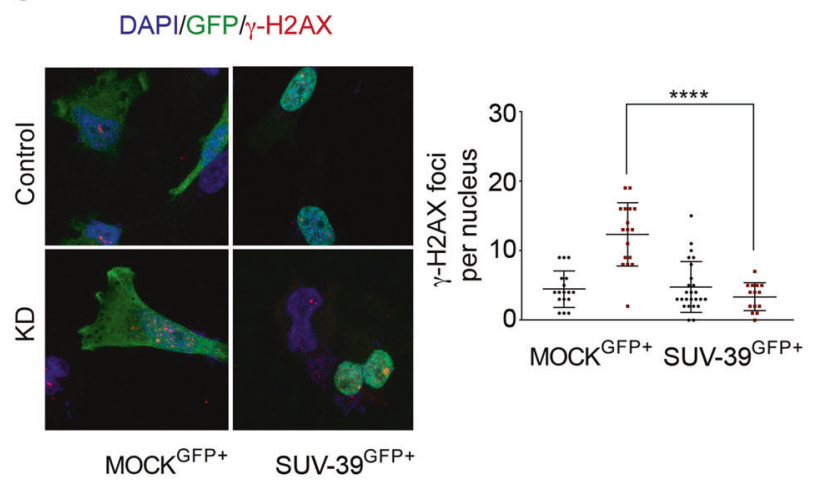

indicated (right panel). $\mathbf{c}$ Cell cycle profile of control and LOXL2 KD cells at $0,2.5,5$, or $7.5 \mathrm{~h}$ after release from a double-thymidine block. Cells were analyzed by fluorescence activated cell sorting (FACS) after propidium iodide staining. d H3S10 phosphorylation levels in control and LOXL2 KD MDA-MB-231 cells were analyzed by western blot. Tubulin was used as a loading control. e Upper panel, MTT assay in control and LOXL2 KD MDA-MB-231 cells; lower panel, colonysurvival assay in control and LOXL2 KD MDA-MB-231 cells. $\gamma$ $\mathrm{H} 2 \mathrm{AX}$ staining and foci quantification are shown by immunofluorescence with the indicated antibody after H1 (f) or SUV-39H1 reinfection (g). MDA-MB-231 cells were infected with control or LOXL2 KD lentivirus, selected with puromycin, and reinfected with GFP $\left(\mathrm{MOCK}^{\mathrm{GFP}+}\right)$, histone 1-GFP $\left(\mathrm{H}^{\mathrm{GFP}+}\right)$ (f) or SUV-39H1-GFP $\left(\mathrm{SUV}-39^{\mathrm{GPP}+}\right)(\mathbf{g})$. Cells were fixed after $24 \mathrm{~h}$. Dot graphs indicate the number of $\gamma$-H2AX foci per GFP-positive cell in each condition. Error bars indicate the SD from at least three independent experiments. $* * P$ $<0.01, * * * P<0.001, * * * * P<0.0001$

histone H1(H1)-GFP expression. Cells were first grown to $70 \%$ confluency and transfected with a mixture of $\mathrm{NaCl}$ $(150 \mathrm{mM})$, a DNA mixture of FUGW-H1-empty vector/ FUGW-H1-GFP (7.5 $\mu \mathrm{g})$, pCMV-VSVG $(1.5 \mu \mathrm{g})$, pMDLg/ pRRE $(4.5 \mu \mathrm{g})$, and pRSV-Rev $(1.5 \mu \mathrm{g})$, and 
Table 2 Genes upregulated in LOXL2 knockdown

\begin{tabular}{|c|c|c|c|c|}
\hline Locus & $\log \mathrm{FC}$ & $P$-Value & FDR & Gene name \\
\hline ENSG00000106541 & 2.557531982 & 0 & & AGR2 \\
\hline ENSG00000185567 & 2.176849204 & $5.15 \mathrm{E}-299$ & $2.25 \mathrm{E}-295$ & AHNAK2 \\
\hline ENSG00000132821 & 2.080483757 & $2.77 \mathrm{E}-280$ & $9.09 \mathrm{E}-277$ & VSTM2L \\
\hline ENSG00000117984 & 1.586454466 & $3.61 \mathrm{E}-273$ & $9.49 \mathrm{E}-270$ & CTSD \\
\hline ENSG00000039139 & 1.913971451 & $1.13 \mathrm{E}-234$ & $1.49 \mathrm{E}-231$ & DNAH5 \\
\hline ENSG00000163083 & 1.827194971 & $3.38 \mathrm{E}-216$ & $4.03 \mathrm{E}-213$ & INHBB \\
\hline ENSG00000184371 & 1.564908962 & $9.87 \mathrm{E}-215$ & $1.08 \mathrm{E}-211$ & CSF1 \\
\hline ENSG00000170373 & 1.445267367 & $7.43 \mathrm{E}-214$ & $7.51 \mathrm{E}-211$ & CST1 \\
\hline ENSG00000183018 & 2.687264202 & $2.37 \mathrm{E}-207$ & $2.22 \mathrm{E}-204$ & SPNS2 \\
\hline ENSG00000148180 & 1.25021061 & $1.35 \mathrm{E}-204$ & $1.18 \mathrm{E}-201$ & GSN \\
\hline ENSG00000187678 & 1.29153331 & $2.72 \mathrm{E}-191$ & $2.23 \mathrm{E}-188$ & SPRY4 \\
\hline ENSG00000109062 & 1.468364079 & $5.45 \mathrm{E}-183$ & $3.97 \mathrm{E}-180$ & SLC9A3R1 \\
\hline ENSG00000124766 & 1.897033792 & $1.36 \mathrm{E}-178$ & $9.39 \mathrm{E}-176$ & SOX4 \\
\hline ENSG00000100994 & 1.081533166 & $2.15 \mathrm{E}-168$ & $1.35 \mathrm{E}-165$ & PYGB \\
\hline ENSG00000181634 & 1.433165343 & $1.64 \mathrm{E}-154$ & $8.26 \mathrm{E}-152$ & TNFSF15 \\
\hline ENSG00000147676 & 1.792602889 & $2.15 \mathrm{E}-153$ & $1.05 \mathrm{E}-150$ & MAL2 \\
\hline ENSG00000107819 & 1.097217673 & $1.35 \mathrm{E}-152$ & $6.33 \mathrm{E}-150$ & SFXN3 \\
\hline ENSG00000167644 & 1.245454774 & $2.18 \mathrm{E}-150$ & $9.88 \mathrm{E}-148$ & C19orf33 \\
\hline ENSG00000172794 & 2.37902321 & $3.41 \mathrm{E}-147$ & $1.49 \mathrm{E}-144$ & RAB37 \\
\hline ENSG00000058085 & 1.09085532 & $1.15 \mathrm{E}-145$ & $4.88 \mathrm{E}-143$ & LAMC2 \\
\hline ENSG00000175130 & 1.074875932 & $2.20 \mathrm{E}-139$ & $9.03 \mathrm{E}-137$ & MARCKSL1 \\
\hline ENSG00000171345 & 1.019257679 & $8.08 \mathrm{E}-137$ & $3.12 \mathrm{E}-134$ & KRT19 \\
\hline ENSG00000167552 & 1.388799485 & $1.60 \mathrm{E}-136$ & $5.82 \mathrm{E}-134$ & TUBA1A \\
\hline ENSG00000132470 & 1.437708599 & $1.07 \mathrm{E}-135$ & $3.79 \mathrm{E}-133$ & ITGB4 \\
\hline ENSG00000008513 & 1.019331762 & $3.84 \mathrm{E}-133$ & $1.29 \mathrm{E}-130$ & ST3GAL1 \\
\hline ENSG00000167642 & 0.963304201 & $1.10 \mathrm{E}-126$ & $3.43 \mathrm{E}-124$ & SPINT2 \\
\hline ENSG00000196154 & 0.94711039 & $2.71 \mathrm{E}-126$ & $8.28 \mathrm{E}-124$ & S100A4 \\
\hline ENSG00000161013 & 1.17382212 & $9.23 \mathrm{E}-123$ & $2.64 \mathrm{E}-120$ & MGAT4B \\
\hline ENSG00000144136 & 0.959244783 & $4.64 \mathrm{E}-122$ & $1.30 \mathrm{E}-119$ & SLC20A1 \\
\hline ENSG00000124145 & 0.935893618 & $1.39 \mathrm{E}-119$ & $3.73 \mathrm{E}-117$ & SDC4 \\
\hline ENSG00000124664 & 1.067904809 & $1.15 \mathrm{E}-118$ & $3.03 \mathrm{E}-116$ & SPDEF \\
\hline ENSG00000108639 & 0.963584447 & $1.29 \mathrm{E}-117$ & $3.32 \mathrm{E}-115$ & SYNGR2 \\
\hline ENSG00000099812 & 2.361113786 & $2.95 \mathrm{E}-117$ & $7.18 \mathrm{E}-115$ & MISP \\
\hline ENSG00000153292 & 1.189984144 & $2.35 \mathrm{E}-112$ & $5.32 \mathrm{E}-110$ & ADGRF1 \\
\hline ENSG00000071054 & 0.884375284 & $1.55 \mathrm{E}-110$ & $3.39 \mathrm{E}-108$ & MAP4K4 \\
\hline ENSG00000163683 & 1.090539229 & $6.92 \mathrm{E}-109$ & $1.49 \mathrm{E}-106$ & SMIM14 \\
\hline ENSG00000069122 & 0.8914899 & $1.23 \mathrm{E}-107$ & $2.48 \mathrm{E}-105$ & ADGRF5 \\
\hline ENSG00000163346 & 1.002881721 & $4.33 \mathrm{E}-107$ & $8.61 \mathrm{E}-105$ & PBXIP1 \\
\hline ENSG00000168032 & 1.546762635 & $6.76 \mathrm{E}-107$ & $1.32 \mathrm{E}-104$ & ENTPD3 \\
\hline ENSG00000010404 & 1.093704162 & $1.94 \mathrm{E}-106$ & $3.74 \mathrm{E}-104$ & IDS \\
\hline ENSG00000026751 & 1.232149717 & $1.10 \mathrm{E}-100$ & $1.95 \mathrm{E}-98$ & SLAMF7 \\
\hline ENSG00000149177 & 0.896255656 & $1.20 \mathrm{E}-98$ & $2.04 \mathrm{E}-96$ & PTPRJ \\
\hline ENSG00000169213 & 2.790765942 & $6.07 \mathrm{E}-98$ & $1.02 \mathrm{E}-95$ & RAB3B \\
\hline ENSG00000148344 & 1.010954162 & $2.71 \mathrm{E}-97$ & $4.51 \mathrm{E}-95$ & PTGES \\
\hline ENSG00000167232 & 1.137723821 & $6.17 \mathrm{E}-97$ & $9.99 \mathrm{E}-95$ & ZNF91 \\
\hline ENSG00000162337 & 0.931691275 & $4.04 \mathrm{E}-96$ & $6.40 \mathrm{E}-94$ & LRP5 \\
\hline ENSG00000013364 & 0.847555049 & $1.19 \mathrm{E}-94$ & $1.87 \mathrm{E}-92$ & MVP \\
\hline ENSG00000143061 & 1.006284582 & $2.26 \mathrm{E}-94$ & $3.49 \mathrm{E}-92$ & IGSF3 \\
\hline ENSG00000119630 & 1.384163447 & $1.38 \mathrm{E}-93$ & $2.11 \mathrm{E}-91$ & PGF \\
\hline ENSG00000138678 & 0.902786259 & $1.84 \mathrm{E}-90$ & $2.65 \mathrm{E}-88$ & GPAT3 \\
\hline ENSG00000125731 & 1.560012238 & $2.27 \mathrm{E}-90$ & $3.24 \mathrm{E}-88$ & SH2D3A \\
\hline ENSG00000101441 & 0.900355704 & $3.67 \mathrm{E}-90$ & $5.18 \mathrm{E}-88$ & CST4 \\
\hline ENSG00000174804 & 1.138994169 & $1.66 \mathrm{E}-89$ & $2.32 \mathrm{E}-87$ & FZD4 \\
\hline ENSG00000135480 & 0.885290783 & $1.96 \mathrm{E}-89$ & $2.71 \mathrm{E}-87$ & KRT7 \\
\hline ENSG00000114554 & 0.782941224 & $6.05 \mathrm{E}-89$ & $8.19 \mathrm{E}-87$ & PLXNA1 \\
\hline ENSG00000131981 & 0.825525944 & $3.76 \mathrm{E}-88$ & $5.04 \mathrm{E}-86$ & LGALS3 \\
\hline ENSG00000205542 & 0.843455136 & $7.86 \mathrm{E}-87$ & $1.02 \mathrm{E}-84$ & TMSB4X \\
\hline ENSG00000118898 & 2.469371901 & $1.48 \mathrm{E}-86$ & $1.91 \mathrm{E}-84$ & PPL \\
\hline ENSG00000114739 & 0.98377701 & $1.49 \mathrm{E}-86$ & $1.91 \mathrm{E}-84$ & ACVR2B \\
\hline ENSG00000172296 & 0.928175781 & $1.83 \mathrm{E}-86$ & $2.31 \mathrm{E}-84$ & SPTLC3 \\
\hline ENSG00000173801 & 0.93691533 & $2.54 \mathrm{E}-86$ & $3.18 \mathrm{E}-84$ & JUP \\
\hline ENSG00000136205 & 0.893027849 & $5.42 \mathrm{E}-85$ & $6.59 \mathrm{E}-83$ & TNS3 \\
\hline ENSG00000078246 & 1.095298427 & $2.01 \mathrm{E}-84$ & $2.40 \mathrm{E}-82$ & TULP3 \\
\hline ENSG00000165757 & 1.886253371 & $7.17 \mathrm{E}-84$ & $8.48 \mathrm{E}-82$ & KIAA1462 \\
\hline ENSG00000158109 & 0.91823575 & $1.48 \mathrm{E}-82$ & $1.69 \mathrm{E}-80$ & TPRG1L \\
\hline ENSG00000115295 & 0.781412289 & $7.32 \mathrm{E}-82$ & $8.29 \mathrm{E}-80$ & CLIP4 \\
\hline
\end{tabular}


Table 2 (continued)

\begin{tabular}{|c|c|c|c|c|}
\hline Locus & $\log \mathrm{FC}$ & $P$-Value & FDR & Gene name \\
\hline ENSG00000151651 & 0.773631649 & $1.07 \mathrm{E}-81$ & $1.20 \mathrm{E}-79$ & ADAM8 \\
\hline ENSG00000183087 & 0.797199229 & $1.18 \mathrm{E}-80$ & $1.32 \mathrm{E}-78$ & GAS6 \\
\hline ENSG00000134531 & 0.763620701 & $1.89 \mathrm{E}-80$ & $2.08 \mathrm{E}-78$ & EMP1 \\
\hline ENSG00000071242 & 1.290957558 & $2.94 \mathrm{E}-80$ & $3.20 \mathrm{E}-78$ & RPS6KA2 \\
\hline ENSG00000074527 & 1.161231478 & $5.89 \mathrm{E}-80$ & $6.29 \mathrm{E}-78$ & NTN4 \\
\hline ENSG00000122359 & 0.732215153 & $8.78 \mathrm{E}-78$ & $9.15 \mathrm{E}-76$ & ANXA11 \\
\hline ENSG00000116478 & 0.727866925 & $1.16 \mathrm{E}-77$ & $1.19 \mathrm{E}-75$ & HDAC1 \\
\hline ENSG00000065613 & 0.732219022 & $1.56 \mathrm{E}-77$ & $1.58 \mathrm{E}-75$ & SLK \\
\hline ENSG00000108846 & 1.870849664 & $1.05 \mathrm{E}-76$ & $1.06 \mathrm{E}-74$ & $\mathrm{ABCC} 3$ \\
\hline ENSG00000196975 & 0.868874265 & $5.81 \mathrm{E}-76$ & $5.73 \mathrm{E}-74$ & ANXA4 \\
\hline ENSG00000165476 & 0.732395071 & $1.31 \mathrm{E}-75$ & $1.27 \mathrm{E}-73$ & REEP3 \\
\hline ENSG00000147251 & 0.950209054 & $2.84 \mathrm{E}-74$ & $2.68 \mathrm{E}-72$ & DOCK11 \\
\hline ENSG00000104081 & 1.619563862 & $1.16 \mathrm{E}-73$ & $1.09 \mathrm{E}-71$ & BMF \\
\hline ENSG00000115107 & 0.833533503 & $1.66 \mathrm{E}-73$ & $1.55 \mathrm{E}-71$ & STEAP3 \\
\hline ENSG00000146072 & 0.740689199 & $2.97 \mathrm{E}-73$ & $2.71 \mathrm{E}-71$ & TNFRSF2 1 \\
\hline ENSG00000118640 & 0.8568247 & $7.92 \mathrm{E}-73$ & $7.18 \mathrm{E}-71$ & VAMP8 \\
\hline ENSG00000198756 & 1.324358789 & $1.14 \mathrm{E}-72$ & $1.02 \mathrm{E}-70$ & COLGALT2 \\
\hline ENSG00000161714 & 0.795194647 & $1.36 \mathrm{E}-72$ & $1.22 \mathrm{E}-70$ & PLCD3 \\
\hline ENSG00000178726 & 0.836487297 & $3.26 \mathrm{E}-72$ & $2.88 \mathrm{E}-70$ & THBD \\
\hline ENSG00000160271 & 0.831966049 & $3.75 \mathrm{E}-72$ & $3.29 \mathrm{E}-70$ & RALGDS \\
\hline ENSG00000102271 & 0.886072448 & $7.99 \mathrm{E}-72$ & $6.95 \mathrm{E}-70$ & KLHL4 \\
\hline ENSG00000112367 & 0.989558706 & $2.33 \mathrm{E}-71$ & $2.01 \mathrm{E}-69$ & FIG4 \\
\hline ENSG00000073849 & 1.156064112 & $7.50 \mathrm{E}-71$ & $6.35 \mathrm{E}-69$ & ST6GAL1 \\
\hline ENSG00000001461 & 0.759211956 & $2.61 \mathrm{E}-70$ & $2.19 \mathrm{E}-68$ & NIPAL3 \\
\hline ENSG00000105778 & 0.716804361 & $7.67 \mathrm{E}-70$ & $6.41 \mathrm{E}-68$ & AVL9 \\
\hline ENSG00000180861 & 2.544226107 & $5.43 \mathrm{E}-26$ & $8.20 \mathrm{E}-25$ & LINC01559 \\
\hline ENSG00000138764 & 0.853001536 & $4.67 \mathrm{E}-69$ & $3.76 \mathrm{E}-67$ & CCNG2 \\
\hline ENSG00000168461 & 0.739918812 & $6.17 \mathrm{E}-69$ & $4.94 \mathrm{E}-67$ & RAB31 \\
\hline ENSG00000165474 & 1.287094496 & $7.37 \mathrm{E}-69$ & $5.87 \mathrm{E}-67$ & GJB2 \\
\hline ENSG00000117472 & 1.402524019 & $3.06 \mathrm{E}-68$ & $2.41 \mathrm{E}-66$ & TSPAN1 \\
\hline ENSG00000124496 & 0.733809105 & $7.91 \mathrm{E}-68$ & $6.04 \mathrm{E}-66$ & TRERF1 \\
\hline ENSG00000171877 & 0.944409394 & $3.72 \mathrm{E}-67$ & $2.73 \mathrm{E}-65$ & FRMD5 \\
\hline ENSG00000126759 & 1.579268489 & $7.91 \mathrm{E}-67$ & $5.74 \mathrm{E}-65$ & CFP \\
\hline ENSG00000124882 & 1.027808793 & $1.06 \mathrm{E}-66$ & $7.64 \mathrm{E}-65$ & EREG \\
\hline ENSG00000102316 & 0.67559959 & $1.29 \mathrm{E}-66$ & $9.23 \mathrm{E}-65$ & MAGED2 \\
\hline ENSG00000075711 & 0.664598245 & $4.29 \mathrm{E}-66$ & $2.99 \mathrm{E}-64$ & DLG1 \\
\hline ENSG00000163435 & 2.261552237 & $1.24 \mathrm{E}-64$ & $8.43 \mathrm{E}-63$ & ELF3 \\
\hline ENSG00000135048 & 0.842294959 & $2.39 \mathrm{E}-64$ & $1.62 \mathrm{E}-62$ & TMEM2 \\
\hline ENSG00000141279 & 0.798544903 & $3.43 \mathrm{E}-64$ & $2.31 \mathrm{E}-62$ & NPEPPS \\
\hline ENSG00000124491 & 1.928383505 & $3.87 \mathrm{E}-64$ & $2.58 \mathrm{E}-62$ & F13A1 \\
\hline ENSG00000136068 & 0.799841098 & $1.04 \mathrm{E}-63$ & $6.88 \mathrm{E}-62$ & FLNB \\
\hline ENSG00000170786 & 1.469743357 & $2.22 \mathrm{E}-63$ & $1.45 \mathrm{E}-61$ & SDR16C5 \\
\hline ENSG00000069702 & 0.968766875 & $2.52 \mathrm{E}-63$ & $1.64 \mathrm{E}-61$ & TGFBR3 \\
\hline ENSG00000069956 & 0.687274901 & $3.83 \mathrm{E}-63$ & $2.48 \mathrm{E}-61$ & MAPK6 \\
\hline ENSG00000183826 & 0.802508399 & $7.36 \mathrm{E}-63$ & $4.72 \mathrm{E}-61$ & BTBD9 \\
\hline ENSG00000137449 & 0.953988726 & $9.13 \mathrm{E}-63$ & $5.82 \mathrm{E}-61$ & CPEB2 \\
\hline ENSG00000160789 & 0.769443927 & $1.58 \mathrm{E}-62$ & $9.90 \mathrm{E}-61$ & LMNA \\
\hline ENSG00000115756 & 0.635371525 & $1.24 \mathrm{E}-60$ & $7.58 \mathrm{E}-59$ & HPCAL1 \\
\hline ENSG00000169439 & 0.774541548 & $2.36 \mathrm{E}-60$ & $1.42 \mathrm{E}-58$ & $\mathrm{SDC} 2$ \\
\hline ENSG00000184564 & 2.470980644 & $2.81 \mathrm{E}-60$ & $1.68 \mathrm{E}-58$ & SLITRK6 \\
\hline ENSG00000143816 & 1.634586486 & $5.68 \mathrm{E}-60$ & $3.36 \mathrm{E}-58$ & WNT9A \\
\hline ENSG00000182093 & 0.87376722 & $7.53 \mathrm{E}-60$ & $4.44 \mathrm{E}-58$ & WRB \\
\hline ENSG00000065054 & 0.668246405 & $9.12 \mathrm{E}-60$ & $5.32 \mathrm{E}-58$ & SLC9A3R2 \\
\hline ENSG00000116701 & 1.117244095 & $1.12 \mathrm{E}-59$ & $6.48 \mathrm{E}-58$ & NCF2 \\
\hline ENSG00000100439 & 0.801911634 & $1.37 \mathrm{E}-59$ & $7.90 \mathrm{E}-58$ & ABHD4 \\
\hline ENSG00000101439 & 0.771824808 & $1.52 \mathrm{E}-59$ & $8.73 \mathrm{E}-58$ & CST3 \\
\hline ENSG00000167779 & 0.981583691 & $1.61 \mathrm{E}-59$ & $9.18 \mathrm{E}-58$ & IGFBP6 \\
\hline ENSG00000075618 & 0.742779504 & $1.63 \mathrm{E}-59$ & $9.25 \mathrm{E}-58$ & FSCN1 \\
\hline ENSG00000178585 & 0.715678973 & $1.83 \mathrm{E}-59$ & $1.03 \mathrm{E}-57$ & CTNNBIP1 \\
\hline ENSG00000088836 & 1.218822163 & $4.54 \mathrm{E}-59$ & $2.53 \mathrm{E}-57$ & SLC4A11 \\
\hline ENSG00000006118 & 0.63601116 & $5.56 \mathrm{E}-59$ & $3.08 \mathrm{E}-57$ & TMEM132A \\
\hline ENSG00000140950 & 0.734048999 & $9.06 \mathrm{E}-59$ & $4.98 \mathrm{E}-57$ & TLDC1 \\
\hline ENSG00000114353 & 0.624135935 & $1.44 \mathrm{E}-58$ & $7.86 \mathrm{E}-57$ & GNAI2 \\
\hline ENSG00000189143 & 0.778650604 & $3.07 \mathrm{E}-58$ & $1.65 \mathrm{E}-56$ & CLDN4 \\
\hline ENSG00000166925 & 0.750896396 & $4.93 \mathrm{E}-58$ & $2.63 \mathrm{E}-56$ & TSC22D4 \\
\hline ENSG00000169991 & 0.777045163 & $5.47 \mathrm{E}-58$ & $2.90 \mathrm{E}-56$ & IFFO2 \\
\hline
\end{tabular}


Table 2 (continued)

\begin{tabular}{|c|c|c|c|c|}
\hline Locus & $\log \mathrm{FC}$ & $P$-Value & FDR & Gene name \\
\hline ENSG00000198910 & 0.697815856 & $1.08 \mathrm{E}-57$ & $5.67 \mathrm{E}-56$ & L1CAM \\
\hline ENSG00000142627 & 0.64537199 & $8.67 \mathrm{E}-57$ & $4.47 \mathrm{E}-55$ & ЕРHA2 \\
\hline ENSG00000119899 & 0.702317857 & $1.04 \mathrm{E}-56$ & $5.35 \mathrm{E}-55$ & SLC17A5 \\
\hline ENSG00000111846 & 0.636366397 & $1.06 \mathrm{E}-56$ & $5.42 \mathrm{E}-55$ & GCNT2 \\
\hline ENSG00000100234 & 0.679728149 & $2.30 \mathrm{E}-56$ & $1.15 \mathrm{E}-54$ & TIMP3 \\
\hline ENSG00000095203 & 0.700479323 & $3.83 \mathrm{E}-56$ & $1.91 \mathrm{E}-54$ & EPB41L4B \\
\hline ENSG00000151276 & 0.736006307 & $6.05 \mathrm{E}-56$ & $3.00 \mathrm{E}-54$ & MAGI1 \\
\hline ENSG00000171310 & 0.607237912 & $1.24 \mathrm{E}-55$ & $6.09 \mathrm{E}-54$ & CHST11 \\
\hline ENSG00000165434 & 0.816777806 & $2.01 \mathrm{E}-55$ & $9.82 \mathrm{E}-54$ & PGM2L1 \\
\hline ENSG00000154930 & 1.233947636 & $5.25 \mathrm{E}-55$ & $2.55 \mathrm{E}-53$ & ACSS1 \\
\hline ENSG00000116285 & 0.720021425 & $7.57 \mathrm{E}-55$ & $3.67 \mathrm{E}-53$ & ERRFI1 \\
\hline ENSG00000106799 & 0.601363577 & $1.04 \mathrm{E}-54$ & $5.00 \mathrm{E}-53$ & TGFBR1 \\
\hline ENSG00000100055 & 1.153795642 & $1.14 \mathrm{E}-54$ & $5.50 \mathrm{E}-53$ & СYTH4 \\
\hline ENSG00000204634 & 0.78091263 & $1.43 \mathrm{E}-54$ & $6.80 \mathrm{E}-53$ & TBC1D8 \\
\hline ENSG00000187764 & 0.934447405 & $3.48 \mathrm{E}-54$ & $1.64 \mathrm{E}-52$ & SEMA4D \\
\hline ENSG00000196611 & 0.697333766 & $6.80 \mathrm{E}-54$ & $3.18 \mathrm{E}-52$ & MMP1 \\
\hline ENSG00000072210 & 0.677662089 & $7.95 \mathrm{E}-54$ & $3.70 \mathrm{E}-52$ & ALDH3A2 \\
\hline ENSG00000166750 & 0.934437232 & $1.24 \mathrm{E}-53$ & $5.75 \mathrm{E}-52$ & SLFN5 \\
\hline ENSG00000160213 & 0.660113084 & $2.58 \mathrm{E}-53$ & $1.19 \mathrm{E}-51$ & CSTB \\
\hline ENSG00000154175 & 1.422923707 & $3.12 \mathrm{E}-53$ & $1.43 \mathrm{E}-51$ & ABI3BP \\
\hline ENSG00000105048 & 0.648949367 & $3.73 \mathrm{E}-53$ & $1.71 \mathrm{E}-51$ & TNNT1 \\
\hline ENSG00000029534 & 0.948107565 & $3.84 \mathrm{E}-53$ & $1.75 \mathrm{E}-51$ & ANK1 \\
\hline ENSG00000163874 & 0.734961316 & $1.42 \mathrm{E}-52$ & $6.44 \mathrm{E}-51$ & ZC3H12A \\
\hline ENSG00000003249 & 1.44442704 & $3.21 \mathrm{E}-52$ & $1.44 \mathrm{E}-50$ & DBNDD1 \\
\hline ENSG00000059804 & 0.834124252 & $4.53 \mathrm{E}-52$ & $2.00 \mathrm{E}-50$ & SLC2A3 \\
\hline ENSG00000120337 & 1.718937071 & $4.63 \mathrm{E}-52$ & $2.04 \mathrm{E}-50$ & TNFSF18 \\
\hline ENSG00000182022 & 0.664899083 & $5.48 \mathrm{E}-52$ & $2.41 \mathrm{E}-50$ & CHST15 \\
\hline ENSG00000188191 & 0.90765776 & $6.61 \mathrm{E}-52$ & $2.88 \mathrm{E}-50$ & PRKAR1B \\
\hline ENSG00000003147 & 1.021914278 & $1.02 \mathrm{E}-51$ & $4.40 \mathrm{E}-50$ & ICA1 \\
\hline ENSG00000018408 & 0.61449093 & $1.18 \mathrm{E}-51$ & $5.09 \mathrm{E}-50$ & WWTR1 \\
\hline ENSG00000188643 & 0.612623576 & $1.72 \mathrm{E}-51$ & $7.37 \mathrm{E}-50$ & S100A16 \\
\hline ENSG00000106070 & 0.622574635 & $2.67 \mathrm{E}-51$ & $1.14 \mathrm{E}-49$ & GRB10 \\
\hline ENSG00000128342 & 0.665706893 & 3.64E-51 & 1.55E-49 & LIF \\
\hline ENSG00000091409 & 0.586574997 & $1.51 \mathrm{E}-50$ & $6.36 \mathrm{E}-49$ & ITGA6 \\
\hline ENSG00000130477 & 1.980813997 & $2.31 \mathrm{E}-50$ & $9.70 \mathrm{E}-49$ & UNC13A \\
\hline ENSG00000059377 & 1.224196279 & $4.24 \mathrm{E}-50$ & $1.76 \mathrm{E}-48$ & TBXAS1 \\
\hline ENSG00000167106 & 0.797725209 & $5.46 \mathrm{E}-50$ & $2.26 \mathrm{E}-48$ & FAM102A \\
\hline ENSG00000011009 & 0.708291693 & $5.65 \mathrm{E}-50$ & $2.33 \mathrm{E}-48$ & LYPLA2 \\
\hline ENSG00000188042 & 0.587843927 & $1.01 \mathrm{E}-49$ & $4.16 \mathrm{E}-48$ & ARL4C \\
\hline ENSG00000106258 & 3.99646604 & $1.07 \mathrm{E}-49$ & $4.39 \mathrm{E}-48$ & CYP3A5 \\
\hline ENSG00000253719 & 0.641918884 & $1.98 \mathrm{E}-49$ & 7.95E-48 & ATXN7L3B \\
\hline ENSG00000198561 & 0.6435595 & $2.03 \mathrm{E}-49$ & $8.11 \mathrm{E}-48$ & CTNND1 \\
\hline ENSG00000196642 & 0.629620891 & $2.44 \mathrm{E}-49$ & $9.74 \mathrm{E}-48$ & RABL6 \\
\hline ENSG00000162909 & 0.627093674 & $4.17 \mathrm{E}-49$ & $1.66 \mathrm{E}-47$ & CAPN2 \\
\hline ENSG00000166046 & 1.014081966 & $1.05 \mathrm{E}-48$ & $4.14 \mathrm{E}-47$ & TCP11L2 \\
\hline ENSG00000106537 & 1.142647315 & $1.06 \mathrm{E}-48$ & $4.16 \mathrm{E}-47$ & TSPAN13 \\
\hline ENSG00000153179 & 0.654407199 & $1.21 \mathrm{E}-48$ & $4.77 \mathrm{E}-47$ & RASSF3 \\
\hline ENSG00000197442 & 0.704516379 & $1.30 \mathrm{E}-48$ & $5.09 \mathrm{E}-47$ & MAP3K5 \\
\hline ENSG00000138119 & 0.641567321 & $6.81 \mathrm{E}-48$ & $2.62 \mathrm{E}-46$ & MYOF \\
\hline ENSG00000185483 & 0.88479338 & $9.18 \mathrm{E}-48$ & $3.51 \mathrm{E}-46$ & ROR1 \\
\hline ENSG00000184363 & 0.800404785 & $1.32 \mathrm{E}-47$ & $5.02 \mathrm{E}-46$ & PKP3 \\
\hline ENSG00000008083 & 0.690696827 & $1.62 \mathrm{E}-47$ & $6.16 \mathrm{E}-46$ & JARID2 \\
\hline ENSG00000179913 & 1.195221395 & $1.63 \mathrm{E}-47$ & $6.18 \mathrm{E}-46$ & B3GNT3 \\
\hline ENSG00000185127 & 0.709916588 & $1.79 \mathrm{E}-47$ & $6.75 \mathrm{E}-46$ & C6orf120 \\
\hline ENSG00000177106 & 0.686183661 & $4.22 \mathrm{E}-47$ & $1.58 \mathrm{E}-45$ & EPS8L2 \\
\hline ENSG00000188015 & 0.884405539 & $4.58 \mathrm{E}-47$ & $1.71 \mathrm{E}-45$ & S100A3 \\
\hline ENSG00000147400 & 0.694830612 & $1.06 \mathrm{E}-46$ & $3.90 \mathrm{E}-45$ & CETN2 \\
\hline ENSG00000143786 & 0.939975104 & $2.19 \mathrm{E}-46$ & $8.05 \mathrm{E}-45$ & $\mathrm{CNIH} 3$ \\
\hline ENSG00000133805 & 0.873822055 & $7.29 \mathrm{E}-46$ & 2.63E-44 & AMPD3 \\
\hline ENSG00000106780 & 0.689300805 & $7.57 \mathrm{E}-46$ & $2.72 \mathrm{E}-44$ & MEGF9 \\
\hline ENSG00000272398 & 1.043194872 & $2.99 \mathrm{E}-45$ & $1.06 \mathrm{E}-43$ & CD24 \\
\hline ENSG00000099998 & 1.065481895 & $6.33 \mathrm{E}-45$ & $2.23 \mathrm{E}-43$ & GGT5 \\
\hline ENSG00000095383 & 0.635665876 & $1.03 \mathrm{E}-44$ & $3.62 \mathrm{E}-43$ & TBC1D2 \\
\hline ENSG00000109436 & 0.653570925 & $1.05 \mathrm{E}-44$ & 3.65E-43 & TBC1D9 \\
\hline ENSG00000198624 & 0.606978398 & $4.62 \mathrm{E}-44$ & $1.58 \mathrm{E}-42$ & CCDC69 \\
\hline ENSG00000153774 & 0.642071127 & $5.74 \mathrm{E}-44$ & $1.96 \mathrm{E}-42$ & CFDP1 \\
\hline
\end{tabular}


Table 2 (continued)

\begin{tabular}{|c|c|c|c|c|}
\hline Locus & $\log \mathrm{FC}$ & $P$-Value & FDR & Gene name \\
\hline ENSG00000130958 & 0.846711873 & $6.23 \mathrm{E}-44$ & $2.11 \mathrm{E}-42$ & SLC35D2 \\
\hline ENSG00000164938 & 0.883485618 & $8.44 \mathrm{E}-44$ & $2.86 \mathrm{E}-42$ & TP53INP1 \\
\hline ENSG00000163947 & 0.753394922 & $9.40 \mathrm{E}-44$ & $3.17 \mathrm{E}-42$ & ARHGEF3 \\
\hline ENSG00000182809 & 1.082621723 & $2.23 \mathrm{E}-43$ & $7.43 \mathrm{E}-42$ & CRIP2 \\
\hline ENSG00000101842 & 2.125670418 & $3.17 \mathrm{E}-43$ & $1.05 \mathrm{E}-41$ & VSIG1 \\
\hline ENSG00000010278 & 0.692915131 & $3.75 \mathrm{E}-43$ & $1.24 \mathrm{E}-41$ & CD9 \\
\hline ENSG00000141753 & 0.652587531 & $4.03 \mathrm{E}-43$ & $1.33 \mathrm{E}-41$ & IGFBP4 \\
\hline ENSG00000071575 & 0.809537681 & $4.69 \mathrm{E}-43$ & $1.53 \mathrm{E}-41$ & TRIB2 \\
\hline ENSG00000041353 & 0.600506668 & $6.85 \mathrm{E}-43$ & $2.23 \mathrm{E}-41$ & RAB27B \\
\hline ENSG00000124942 & 0.878776788 & $8.14 \mathrm{E}-43$ & $2.64 \mathrm{E}-41$ & AHNAK \\
\hline ENSG00000130702 & 0.830408106 & $1.66 \mathrm{E}-42$ & $5.34 \mathrm{E}-41$ & LAMA5 \\
\hline ENSG00000106605 & 0.707072414 & $1.69 \mathrm{E}-42$ & $5.40 \mathrm{E}-41$ & BLVRA \\
\hline ENSG00000160211 & 0.671328183 & $1.71 \mathrm{E}-42$ & $5.47 \mathrm{E}-41$ & G6PD \\
\hline ENSG00000153395 & 0.667145468 & $1.73 \mathrm{E}-42$ & $5.50 \mathrm{E}-41$ & LPCAT1 \\
\hline ENSG00000183778 & 1.193744584 & $3.32 \mathrm{E}-42$ & $1.05 \mathrm{E}-40$ & B3GALT5 \\
\hline ENSG00000131389 & 0.90704002 & $2.13 \mathrm{E}-41$ & $6.66 \mathrm{E}-40$ & SLC6A6 \\
\hline ENSG00000184117 & 0.709961941 & $2.66 \mathrm{E}-41$ & $8.24 \mathrm{E}-40$ & NIPSNAP1 \\
\hline ENSG00000258947 & 1.044442933 & $2.99 \mathrm{E}-41$ & $9.27 \mathrm{E}-40$ & TUBB3 \\
\hline ENSG00000180667 & 0.586841506 & $3.12 \mathrm{E}-41$ & $9.64 \mathrm{E}-40$ & YOD1 \\
\hline ENSG00000157227 & 0.607608231 & $3.40 \mathrm{E}-41$ & $1.05 \mathrm{E}-39$ & MMP14 \\
\hline ENSG00000184557 & 0.61191017 & $1.37 \mathrm{E}-40$ & $4.18 \mathrm{E}-39$ & SOCS3 \\
\hline ENSG00000100106 & 0.606368936 & $2.00 \mathrm{E}-40$ & $6.06 \mathrm{E}-39$ & TRIOBP \\
\hline ENSG00000165233 & 0.647236635 & $2.07 \mathrm{E}-40$ & $6.24 \mathrm{E}-39$ & CARD19 \\
\hline ENSG00000126458 & 0.806490749 & $2.10 \mathrm{E}-40$ & $6.33 \mathrm{E}-39$ & RRAS \\
\hline ENSG00000152217 & 1.432604587 & $3.42 \mathrm{E}-40$ & $1.01 \mathrm{E}-38$ & SETBP1 \\
\hline ENSG00000157796 & 0.64712781 & $4.11 \mathrm{E}-40$ & $1.22 \mathrm{E}-38$ & WDR19 \\
\hline ENSG00000118960 & 0.794746258 & $4.94 \mathrm{E}-40$ & $1.46 \mathrm{E}-38$ & HS1BP3 \\
\hline ENSG00000078269 & 0.598064657 & $1.32 \mathrm{E}-39$ & $3.86 \mathrm{E}-38$ & SYNJ2 \\
\hline ENSG00000133138 & 1.209003045 & $1.35 \mathrm{E}-39$ & $3.94 \mathrm{E}-38$ & TBC1D8B \\
\hline ENSG00000088387 & 0.585401778 & $1.40 \mathrm{E}-39$ & $4.07 \mathrm{E}-38$ & DOCK9 \\
\hline ENSG00000251191 & 2.434425725 & $2.57 \mathrm{E}-38$ & $6.95 \mathrm{E}-37$ & LINC00589 \\
\hline ENSG00000203697 & 4.744840946 & $1.53 \mathrm{E}-39$ & $4.43 \mathrm{E}-38$ & CAPN8 \\
\hline ENSG00000158769 & 0.653902818 & $1.73 \mathrm{E}-39$ & $5.00 \mathrm{E}-38$ & F11R \\
\hline ENSG00000062282 & 0.689520114 & $2.55 \mathrm{E}-39$ & $7.25 \mathrm{E}-38$ & DGAT2 \\
\hline ENSG00000184497 & 0.978103665 & $2.65 \mathrm{E}-39$ & $7.52 \mathrm{E}-38$ & TMEM255B \\
\hline ENSG00000149948 & 0.723373492 & $2.80 \mathrm{E}-39$ & $7.93 \mathrm{E}-38$ & HMGA2 \\
\hline ENSG00000148730 & 0.588607271 & $3.00 \mathrm{E}-39$ & $8.47 \mathrm{E}-38$ & EIF4EBP2 \\
\hline ENSG00000117983 & 2.62243558 & $9.23 \mathrm{E}-39$ & $2.56 \mathrm{E}-37$ & MUC5B \\
\hline ENSG00000105514 & 0.898143653 & $1.15 \mathrm{E}-38$ & $3.16 \mathrm{E}-37$ & RAB3D \\
\hline ENSG00000169432 & 1.168069383 & $1.36 \mathrm{E}-38$ & $3.72 \mathrm{E}-37$ & SCN9A \\
\hline ENSG00000006534 & 0.83694272 & $1.56 \mathrm{E}-38$ & $4.27 \mathrm{E}-37$ & ALDH3B1 \\
\hline ENSG00000074219 & 0.725674533 & $1.92 \mathrm{E}-38$ & $5.24 \mathrm{E}-37$ & TEAD2 \\
\hline ENSG00000255471 & 1.5585602 & $4.89 \mathrm{E}-14$ & $3.52 \mathrm{E}-13$ & AP001528.2 \\
\hline ENSG00000005238 & 0.737875746 & $2.60 \mathrm{E}-38$ & $7.02 \mathrm{E}-37$ & FAM214B \\
\hline ENSG00000205426 & 0.859387072 & $6.01 \mathrm{E}-38$ & $1.59 \mathrm{E}-36$ & KRT81 \\
\hline ENSG00000258088 & 1.553544167 & $6.65 \mathrm{E}-19$ & $6.78 \mathrm{E}-18$ & AC078820.1 \\
\hline ENSG00000138162 & 0.62551312 & $7.91 \mathrm{E}-38$ & $2.08 \mathrm{E}-36$ & TACC2 \\
\hline ENSG00000141524 & 0.709114969 & $8.26 \mathrm{E}-38$ & $2.17 \mathrm{E}-36$ & TMC6 \\
\hline ENSG00000170271 & 2.264074711 & $8.41 \mathrm{E}-38$ & $2.20 \mathrm{E}-36$ & FAXDC2 \\
\hline ENSG00000136720 & 0.700335625 & $8.54 \mathrm{E}-38$ & $2.23 \mathrm{E}-36$ & HS6ST1 \\
\hline ENSG00000162591 & 1.561892509 & $9.75 \mathrm{E}-38$ & $2.53 \mathrm{E}-36$ & MEGF6 \\
\hline ENSG00000173334 & 0.641809108 & $1.16 \mathrm{E}-37$ & $3.02 \mathrm{E}-36$ & TRIB1 \\
\hline ENSG00000110871 & 0.728862751 & $2.35 \mathrm{E}-37$ & $6.03 \mathrm{E}-36$ & COQ5 \\
\hline ENSG00000150471 & 1.085455968 & $2.61 \mathrm{E}-37$ & $6.68 \mathrm{E}-36$ & ADGRL3 \\
\hline ENSG00000133985 & 1.071387544 & $2.67 \mathrm{E}-37$ & $6.82 \mathrm{E}-36$ & TTC9 \\
\hline ENSG00000165806 & 0.615818489 & $3.03 \mathrm{E}-37$ & $7.70 \mathrm{E}-36$ & CASP7 \\
\hline ENSG00000159733 & 0.690251958 & $3.10 \mathrm{E}-37$ & $7.86 \mathrm{E}-36$ & ZFYVE28 \\
\hline ENSG00000125798 & 0.586210217 & $3.58 \mathrm{E}-37$ & $9.07 \mathrm{E}-36$ & FOXA2 \\
\hline ENSG00000146278 & 0.580483004 & $5.25 \mathrm{E}-37$ & $1.32 \mathrm{E}-35$ & PNRC1 \\
\hline ENSG00000131746 & 2.387958603 & $3.96 \mathrm{E}-36$ & $9.78 \mathrm{E}-35$ & TNS4 \\
\hline ENSG00000137767 & 0.667955094 & $5.74 \mathrm{E}-36$ & $1.40 \mathrm{E}-34$ & SQOR \\
\hline ENSG00000054277 & 1.0616567 & $6.32 \mathrm{E}-36$ & $1.54 \mathrm{E}-34$ & OPN3 \\
\hline ENSG00000116016 & 0.598281377 & $1.05 \mathrm{E}-35$ & $2.53 \mathrm{E}-34$ & EPAS1 \\
\hline ENSG00000151690 & 0.658340738 & $1.15 \mathrm{E}-35$ & $2.77 \mathrm{E}-34$ & MFSD6 \\
\hline ENSG00000168487 & 0.664252835 & $1.26 \mathrm{E}-35$ & $3.02 \mathrm{E}-34$ & BMP1 \\
\hline ENSG00000144642 & 0.724358105 & $2.62 \mathrm{E}-35$ & $6.20 \mathrm{E}-34$ & RBMS3 \\
\hline
\end{tabular}


Table 2 (continued)

\begin{tabular}{|c|c|c|c|c|}
\hline Locus & $\log \mathrm{FC}$ & $P$-Value & FDR & Gene name \\
\hline ENSG00000146386 & 0.582324614 & $3.06 \mathrm{E}-35$ & $7.20 \mathrm{E}-34$ & ABRACL \\
\hline ENSG00000109321 & 0.85796308 & $6.09 \mathrm{E}-35$ & $1.42 \mathrm{E}-33$ & AREG \\
\hline ENSG00000185033 & 0.613573094 & $7.11 \mathrm{E}-35$ & $1.65 \mathrm{E}-33$ & SEMA4B \\
\hline ENSG00000124143 & 1.002818698 & $1.31 \mathrm{E}-34$ & $3.02 \mathrm{E}-33$ & ARHGAP40 \\
\hline ENSG00000188766 & 1.007070905 & $1.37 \mathrm{E}-34$ & $3.15 \mathrm{E}-33$ & SPRED3 \\
\hline ENSG00000143198 & 0.665742779 & $1.45 \mathrm{E}-34$ & $3.31 \mathrm{E}-33$ & MGST3 \\
\hline ENSG00000138772 & 0.618561607 & $1.92 \mathrm{E}-34$ & $4.38 \mathrm{E}-33$ & ANXA3 \\
\hline ENSG00000153208 & 0.65916003 & $2.75 \mathrm{E}-34$ & $6.24 \mathrm{E}-33$ & MERTK \\
\hline ENSG00000105379 & 0.650414245 & $3.72 \mathrm{E}-34$ & $8.40 \mathrm{E}-33$ & ETFB \\
\hline ENSG00000174600 & 1.152413998 & $4.99 \mathrm{E}-34$ & $1.12 \mathrm{E}-32$ & CMKLR1 \\
\hline ENSG00000143878 & 0.677873378 & $6.11 \mathrm{E}-34$ & $1.36 \mathrm{E}-32$ & RHOB \\
\hline ENSG00000129636 & 0.586007283 & $7.21 \mathrm{E}-34$ & $1.60 \mathrm{E}-32$ & ITFG1 \\
\hline ENSG00000196358 & 1.157286665 & $7.31 \mathrm{E}-34$ & $1.62 \mathrm{E}-32$ & NTNG2 \\
\hline ENSG00000074855 & 0.727005968 & $1.29 \mathrm{E}-33$ & $2.85 \mathrm{E}-32$ & ANO8 \\
\hline ENSG00000179869 & 1.478231849 & $1.76 \mathrm{E}-33$ & $3.84 \mathrm{E}-32$ & ABCA13 \\
\hline ENSG00000164379 & 0.688194901 & $2.27 \mathrm{E}-33$ & $4.92 \mathrm{E}-32$ & FOXQ1 \\
\hline ENSG00000082458 & 0.603787281 & $4.54 \mathrm{E}-33$ & $9.68 \mathrm{E}-32$ & DLG3 \\
\hline ENSG00000186106 & 0.614744197 & $1.44 \mathrm{E}-32$ & $3.01 \mathrm{E}-31$ & ANKRD46 \\
\hline ENSG00000127863 & 0.807228706 & $1.48 \mathrm{E}-32$ & $3.09 \mathrm{E}-31$ & TNFRSF19 \\
\hline ENSG00000007384 & 0.675083802 & $1.79 \mathrm{E}-32$ & $3.71 \mathrm{E}-31$ & RHBDF1 \\
\hline ENSG00000175938 & 0.991028089 & $1.90 \mathrm{E}-32$ & $3.92 \mathrm{E}-31$ & ORAI3 \\
\hline ENSG00000258245 & 1.54291117 & $1.93 \mathrm{E}-09$ & $9.27 \mathrm{E}-09$ & RPL10P13 \\
\hline ENSG00000032742 & 0.85419356 & $4.05 \mathrm{E}-32$ & $8.32 \mathrm{E}-31$ & IFT88 \\
\hline ENSG00000229512 & 1.425346349 & $8.83 \mathrm{E}-13$ & $5.81 \mathrm{E}-12$ & AC068580.1 \\
\hline ENSG00000187164 & 0.600256967 & $5.52 \mathrm{E}-32$ & $1.12 \mathrm{E}-30$ & SHTN1 \\
\hline ENSG00000154027 & 1.341272323 & $9.88 \mathrm{E}-32$ & $1.99 \mathrm{E}-30$ & AK5 \\
\hline ENSG00000101846 & 0.734435125 & $1.11 \mathrm{E}-31$ & $2.23 \mathrm{E}-30$ & STS \\
\hline ENSG00000206190 & 0.787741284 & $1.70 \mathrm{E}-31$ & $3.36 \mathrm{E}-30$ & ATP10A \\
\hline ENSG00000160255 & 0.651457256 & $2.24 \mathrm{E}-31$ & $4.40 \mathrm{E}-30$ & ITGB2 \\
\hline ENSG00000101213 & 0.990447452 & $4.59 \mathrm{E}-31$ & $8.91 \mathrm{E}-30$ & PTK6 \\
\hline ENSG00000198286 & 0.883579653 & $5.70 \mathrm{E}-31$ & $1.10 \mathrm{E}-29$ & CARD11 \\
\hline ENSG00000106066 & 0.619864757 & $7.17 \mathrm{E}-31$ & $1.38 \mathrm{E}-29$ & CPVL \\
\hline ENSG00000115919 & 0.677292783 & $8.14 \mathrm{E}-31$ & $1.56 \mathrm{E}-29$ & KYNU \\
\hline ENSG00000064787 & 4.909061593 & $9.51 \mathrm{E}-31$ & $1.81 \mathrm{E}-29$ & BCAS1 \\
\hline ENSG00000144218 & 0.616205371 & $1.12 \mathrm{E}-30$ & $2.13 \mathrm{E}-29$ & AFF3 \\
\hline ENSG00000123095 & 0.933963389 & $1.93 \mathrm{E}-30$ & $3.62 \mathrm{E}-29$ & BHLHE41 \\
\hline ENSG00000148671 & 1.02789778 & $2.52 \mathrm{E}-30$ & $4.69 \mathrm{E}-29$ & ADIRF \\
\hline ENSG00000258077 & 1.387892381 & $1.19 \mathrm{E}-21$ & $1.42 \mathrm{E}-20$ & AC078923.1 \\
\hline ENSG00000125534 & 0.590391835 & $7.90 \mathrm{E}-30$ & $1.44 \mathrm{E}-28$ & PPDPF \\
\hline ENSG00000137501 & 0.668063928 & $1.04 \mathrm{E}-29$ & $1.88 \mathrm{E}-28$ & SYTL2 \\
\hline ENSG00000106351 & 0.728533997 & $1.04 \mathrm{E}-29$ & $1.88 \mathrm{E}-28$ & AGFG2 \\
\hline ENSG00000185585 & 0.755238419 & $1.08 \mathrm{E}-29$ & $1.95 \mathrm{E}-28$ & OLFML2A \\
\hline ENSG00000178951 & 0.587643267 & $1.31 \mathrm{E}-29$ & $2.35 \mathrm{E}-28$ & ZBTB7A \\
\hline ENSG00000111110 & 0.952754467 & $1.54 \mathrm{E}-29$ & $2.76 \mathrm{E}-28$ & PPM1H \\
\hline ENSG00000047056 & 0.582933075 & $2.95 \mathrm{E}-29$ & $5.19 \mathrm{E}-28$ & WDR37 \\
\hline ENSG00000227039 & 1.384680791 & $6.64 \mathrm{E}-17$ & $5.90 \mathrm{E}-16$ & ITGB2-AS1 \\
\hline ENSG00000102096 & 0.607774377 & $7.04 \mathrm{E}-29$ & $1.21 \mathrm{E}-27$ & PIM2 \\
\hline ENSG00000158106 & 0.851822142 & $1.01 \mathrm{E}-28$ & $1.74 \mathrm{E}-27$ & RHPN1 \\
\hline ENSG00000133943 & 0.621897984 & $1.44 \mathrm{E}-28$ & $2.45 \mathrm{E}-27$ & C14orf159 \\
\hline ENSG00000132274 & 0.927124554 & $1.44 \mathrm{E}-28$ & $2.46 \mathrm{E}-27$ & TRIM22 \\
\hline ENSG00000167565 & 0.59119894 & $2.89 \mathrm{E}-28$ & $4.87 \mathrm{E}-27$ & SERTAD3 \\
\hline ENSG00000146416 & 0.722563478 & $9.98 \mathrm{E}-28$ & $1.64 \mathrm{E}-26$ & AIG1 \\
\hline ENSG00000102934 & 1.156498397 & $3.17 \mathrm{E}-27$ & $5.08 \mathrm{E}-26$ & PLLP \\
\hline ENSG00000157613 & 0.66005375 & $9.58 \mathrm{E}-27$ & $1.50 \mathrm{E}-25$ & CREB3L1 \\
\hline ENSG00000139636 & 0.759504331 & $1.05 \mathrm{E}-26$ & $1.64 \mathrm{E}-25$ & LMBR1L \\
\hline ENSG00000124313 & 0.956039086 & $1.06 \mathrm{E}-26$ & $1.65 \mathrm{E}-25$ & IQSEC2 \\
\hline ENSG00000167778 & 0.584757438 & $1.37 \mathrm{E}-26$ & $2.12 \mathrm{E}-25$ & SPRYD3 \\
\hline ENSG00000117305 & 0.692063114 & $2.15 \mathrm{E}-26$ & $3.30 \mathrm{E}-25$ & HMGCL \\
\hline ENSG00000177854 & 1.052435249 & $3.25 \mathrm{E}-26$ & $4.95 \mathrm{E}-25$ & TMEM187 \\
\hline ENSG00000175505 & 0.659053782 & $3.46 \mathrm{E}-26$ & $5.27 \mathrm{E}-25$ & CLCF1 \\
\hline ENSG00000135596 & 0.588183448 & $4.27 \mathrm{E}-26$ & $6.47 \mathrm{E}-25$ & MICAL1 \\
\hline ENSG00000259974 & 1.265560114 & $1.32 \mathrm{E}-08$ & $5.80 \mathrm{E}-08$ & LINC00261 \\
\hline ENSG00000039068 & 1.784191229 & $5.61 \mathrm{E}-26$ & $8.46 \mathrm{E}-25$ & CDH1 \\
\hline ENSG00000139044 & 2.17079755 & $6.20 \mathrm{E}-26$ & $9.34 \mathrm{E}-25$ & B4GALNT3 \\
\hline ENSG00000160446 & 0.580686241 & $8.53 \mathrm{E}-26$ & $1.28 \mathrm{E}-24$ & ZDHHC12 \\
\hline ENSG00000112679 & 0.651511201 & $1.02 \mathrm{E}-25$ & $1.53 \mathrm{E}-24$ & DUSP22 \\
\hline
\end{tabular}


Table 2 (continued)

\begin{tabular}{|c|c|c|c|c|}
\hline Locus & $\log \mathrm{FC}$ & $P$-Value & FDR & Gene name \\
\hline ENSG00000158292 & 0.659371473 & $1.14 \mathrm{E}-25$ & $1.69 \mathrm{E}-24$ & GPR153 \\
\hline ENSG00000167470 & 0.746743002 & $1.27 \mathrm{E}-25$ & $1.88 \mathrm{E}-24$ & MIDN \\
\hline ENSG00000100246 & 0.975110479 & $4.43 \mathrm{E}-25$ & $6.45 \mathrm{E}-24$ & DNAL4 \\
\hline ENSG00000116117 & 0.702904005 & $5.86 \mathrm{E}-25$ & $8.50 \mathrm{E}-24$ & PARD3B \\
\hline ENSG00000100290 & 1.23971931 & $7.52 \mathrm{E}-25$ & $1.08 \mathrm{E}-23$ & BIK \\
\hline ENSG00000165949 & 0.802081907 & $1.33 \mathrm{E}-24$ & $1.89 \mathrm{E}-23$ & IFI27 \\
\hline ENSG00000169083 & 0.656064782 & $1.91 \mathrm{E}-24$ & $2.68 \mathrm{E}-23$ & $\mathrm{AR}$ \\
\hline ENSG00000109610 & 0.77544685 & $2.24 \mathrm{E}-24$ & $3.13 \mathrm{E}-23$ & SOD3 \\
\hline ENSG00000140931 & 0.704129083 & $2.81 \mathrm{E}-24$ & $3.90 \mathrm{E}-23$ & СMTM3 \\
\hline ENSG00000152128 & 0.697760811 & $3.49 \mathrm{E}-24$ & $4.83 \mathrm{E}-23$ & TMEM163 \\
\hline ENSG00000124406 & 0.610126555 & $4.99 \mathrm{E}-24$ & $6.83 \mathrm{E}-23$ & ATP8A1 \\
\hline ENSG00000106069 & 1.002738235 & $6.13 \mathrm{E}-24$ & $8.36 \mathrm{E}-23$ & CHN2 \\
\hline ENSG00000144455 & 0.66426688 & $6.74 \mathrm{E}-24$ & $9.15 \mathrm{E}-23$ & SUMF1 \\
\hline ENSG00000163898 & 0.637729579 & $7.68 \mathrm{E}-24$ & $1.04 \mathrm{E}-22$ & LIPH \\
\hline ENSG00000120306 & 0.686361467 & $7.96 \mathrm{E}-24$ & $1.07 \mathrm{E}-22$ & CYSTM1 \\
\hline ENSG00000171992 & 0.821352578 & $8.82 \mathrm{E}-24$ & $1.19 \mathrm{E}-22$ & SYNPO \\
\hline ENSG00000275342 & 0.707225979 & $8.83 \mathrm{E}-24$ & $1.19 \mathrm{E}-22$ & PRAG1 \\
\hline ENSG00000140022 & 1.275607654 & $1.75 \mathrm{E}-23$ & $2.33 \mathrm{E}-22$ & STON2 \\
\hline ENSG00000006453 & 0.608949037 & $3.37 \mathrm{E}-23$ & $4.41 \mathrm{E}-22$ & BAIAP2L1 \\
\hline ENSG00000102547 & 0.7411813 & $3.62 \mathrm{E}-23$ & $4.72 \mathrm{E}-22$ & CAB39L \\
\hline ENSG00000162105 & 1.018126629 & $3.82 \mathrm{E}-23$ & $4.98 \mathrm{E}-22$ & SHANK2 \\
\hline ENSG00000150782 & 0.667724476 & $4.97 \mathrm{E}-23$ & $6.41 \mathrm{E}-22$ & IL18 \\
\hline ENSG00000063660 & 0.62014441 & $5.38 \mathrm{E}-23$ & $6.92 \mathrm{E}-22$ & GPC1 \\
\hline ENSG00000162869 & 0.633902575 & $6.97 \mathrm{E}-23$ & $8.91 \mathrm{E}-22$ & PPP1R21 \\
\hline ENSG00000065361 & 0.802225109 & $7.32 \mathrm{E}-23$ & $9.35 \mathrm{E}-22$ & ERBB3 \\
\hline ENSG00000081189 & 0.666188328 & $7.32 \mathrm{E}-23$ & $9.35 \mathrm{E}-22$ & MEF2C \\
\hline ENSG00000122176 & 1.592831251 & $2.05 \mathrm{E}-22$ & $2.55 \mathrm{E}-21$ & FMOD \\
\hline ENSG00000069812 & 1.132970411 & $2.34 \mathrm{E}-22$ & $2.90 \mathrm{E}-21$ & HES2 \\
\hline ENSG00000146530 & 0.946186656 & $2.74 \mathrm{E}-22$ & $3.39 \mathrm{E}-21$ & VWDE \\
\hline ENSG00000249279 & 1.258589847 & $3.01 \mathrm{E}-07$ & $1.13 \mathrm{E}-06$ & LINC02057 \\
\hline ENSG00000151715 & 1.313755414 & $4.48 \mathrm{E}-22$ & $5.44 \mathrm{E}-21$ & TMEM45B \\
\hline ENSG00000100031 & 0.782239579 & $6.38 \mathrm{E}-22$ & $7.72 \mathrm{E}-21$ & GGT1 \\
\hline ENSG00000225339 & 1.250563168 & $1.16 \mathrm{E}-21$ & $1.38 \mathrm{E}-20$ & AL354740.1 \\
\hline ENSG00000230439 & 1.230428653 & $3.86 \mathrm{E}-30$ & $7.17 \mathrm{E}-29$ & AL512488.1 \\
\hline ENSG00000105429 & 0.589326615 & $1.81 \mathrm{E}-21$ & $2.15 \mathrm{E}-20$ & MEGF8 \\
\hline ENSG00000143344 & 0.598376949 & $1.82 \mathrm{E}-21$ & $2.16 \mathrm{E}-20$ & RGL1 \\
\hline ENSG00000114805 & 0.907966339 & $2.84 \mathrm{E}-21$ & $3.32 \mathrm{E}-20$ & PLCH1 \\
\hline ENSG00000105856 & 0.586467521 & $3.20 \mathrm{E}-21$ & $3.71 \mathrm{E}-20$ & HBP1 \\
\hline ENSG00000188153 & 0.668030888 & 3.63E-21 & $4.19 \mathrm{E}-20$ & COL4A5 \\
\hline ENSG00000198513 & 0.787106569 & $3.85 \mathrm{E}-21$ & $4.44 \mathrm{E}-20$ & ATL1 \\
\hline ENSG00000113721 & 1.215644215 & $4.56 \mathrm{E}-21$ & $5.25 \mathrm{E}-20$ & PDGFRB \\
\hline ENSG00000183828 & 0.751113819 & $4.82 \mathrm{E}-21$ & $5.54 \mathrm{E}-20$ & NUDT14 \\
\hline ENSG00000181722 & 0.807166236 & $5.79 \mathrm{E}-21$ & $6.63 \mathrm{E}-20$ & ZBTB20 \\
\hline ENSG00000164976 & 0.825285155 & $6.78 \mathrm{E}-21$ & $7.69 \mathrm{E}-20$ & KIAA1161 \\
\hline ENSG00000148737 & 0.823410224 & $7.52 \mathrm{E}-21$ & $8.51 \mathrm{E}-20$ & TCF7L2 \\
\hline ENSG00000167861 & 0.647865569 & $8.61 \mathrm{E}-21$ & $9.72 \mathrm{E}-20$ & HID1 \\
\hline ENSG00000188747 & 1.012209238 & $1.04 \mathrm{E}-20$ & $1.17 \mathrm{E}-19$ & NOXA1 \\
\hline ENSG00000159314 & 0.882984133 & $1.11 \mathrm{E}-20$ & $1.25 \mathrm{E}-19$ & ARHGAP27 \\
\hline ENSG00000089723 & 0.80542898 & $1.35 \mathrm{E}-20$ & $1.51 \mathrm{E}-19$ & OTUB2 \\
\hline ENSG00000088899 & 0.649788574 & $1.38 \mathrm{E}-20$ & $1.55 \mathrm{E}-19$ & LZTS3 \\
\hline ENSG00000154864 & 0.783916945 & $2.24 \mathrm{E}-20$ & $2.49 \mathrm{E}-19$ & $\mathrm{PIEZO} 2$ \\
\hline ENSG00000122515 & 0.582145115 & $2.89 \mathrm{E}-20$ & $3.19 \mathrm{E}-19$ & ZMIZ2 \\
\hline ENSG00000260461 & 1.216719543 & 3.67E-09 & $1.72 \mathrm{E}-08$ & AL133355.1 \\
\hline ENSG00000167105 & 0.61082311 & $3.72 \mathrm{E}-20$ & $4.07 \mathrm{E}-19$ & TMEM92 \\
\hline ENSG00000198429 & 0.697252261 & $3.90 \mathrm{E}-20$ & $4.26 \mathrm{E}-19$ & ZNF69 \\
\hline ENSG00000102452 & 0.765799284 & $4.92 \mathrm{E}-20$ & $5.35 \mathrm{E}-19$ & NALCN \\
\hline ENSG00000108375 & 1.363173418 & $7.55 \mathrm{E}-20$ & $8.14 \mathrm{E}-19$ & RNF43 \\
\hline ENSG00000218014 & 1.189647936 & $1.64 \mathrm{E}-08$ & $7.14 \mathrm{E}-08$ & KRT19P1 \\
\hline ENSG00000133056 & 1.110173291 & $1.42 \mathrm{E}-19$ & $1.51 \mathrm{E}-18$ & PIK3C2B \\
\hline ENSG00000142347 & 1.005039505 & $1.63 \mathrm{E}-19$ & $1.74 \mathrm{E}-18$ & MYO1F \\
\hline ENSG00000267284 & 1.148059389 & $1.95 \mathrm{E}-09$ & $9.39 \mathrm{E}-09$ & AC022031.2 \\
\hline ENSG00000119943 & 0.668952392 & $1.86 \mathrm{E}-19$ & $1.96 \mathrm{E}-18$ & PYROXD2 \\
\hline ENSG00000106003 & 0.591285678 & $1.90 \mathrm{E}-19$ & $2.00 \mathrm{E}-18$ & LFNG \\
\hline ENSG00000240583 & 1.981897469 & $2.32 \mathrm{E}-19$ & $2.43 \mathrm{E}-18$ & $\mathrm{AQP1}$ \\
\hline ENSG00000280303 & 1.116972088 & $5.78 \mathrm{E}-09$ & $2.65 \mathrm{E}-08$ & AC067931.2 \\
\hline ENSG00000125772 & 0.588258566 & $3.68 \mathrm{E}-19$ & $3.83 \mathrm{E}-18$ & GPCPD1 \\
\hline
\end{tabular}


Table 2 (continued)

\begin{tabular}{|c|c|c|c|c|}
\hline Locus & $\log \mathrm{FC}$ & $P$-Value & FDR & Gene name \\
\hline ENSG00000185189 & 0.713266249 & $6.53 \mathrm{E}-19$ & $6.67 \mathrm{E}-18$ & NRBP2 \\
\hline ENSG00000243629 & 1.089891447 & $5.78 \mathrm{E}-11$ & $3.26 \mathrm{E}-10$ & LINC00880 \\
\hline ENSG00000136859 & 0.625902802 & $1.32 \mathrm{E}-18$ & $1.33 \mathrm{E}-17$ & ANGPTL2 2 \\
\hline ENSG00000170412 & 1.064761214 & $1.47 \mathrm{E}-18$ & $1.48 \mathrm{E}-17$ & GPRC5C \\
\hline ENSG00000115525 & 0.786519457 & $1.70 \mathrm{E}-18$ & $1.70 \mathrm{E}-17$ & ST3GAL5 \\
\hline ENSG00000108551 & 0.634579806 & $2.01 \mathrm{E}-18$ & $1.99 \mathrm{E}-17$ & RASD1 \\
\hline ENSG00000260877 & 1.040510062 & $3.58 \mathrm{E}-10$ & 1.85E-09 & AP005233.2 \\
\hline ENSG00000111452 & 0.797580152 & $3.43 \mathrm{E}-18$ & $3.34 \mathrm{E}-17$ & ADGRD1 \\
\hline ENSG00000164010 & 0.671003676 & $4.05 \mathrm{E}-18$ & $3.92 \mathrm{E}-17$ & ERMAP \\
\hline ENSG00000267013 & 1.031866824 & $1.81 \mathrm{E}-19$ & $1.91 \mathrm{E}-18$ & LINC01929 \\
\hline ENSG00000196517 & 0.601022218 & $7.40 \mathrm{E}-18$ & $7.02 \mathrm{E}-17$ & SLC6A9 \\
\hline ENSG00000184985 & 0.953793323 & $8.27 \mathrm{E}-18$ & $7.82 \mathrm{E}-17$ & SORCS2 \\
\hline ENSG00000073350 & 1.015768255 & $9.33 \mathrm{E}-18$ & $8.78 \mathrm{E}-17$ & LLGL2 \\
\hline ENSG00000160181 & 0.979528456 & $9.78 \mathrm{E}-18$ & $9.19 \mathrm{E}-17$ & TFF2 \\
\hline ENSG00000170214 & 0.985557139 & $1.03 \mathrm{E}-17$ & $9.71 \mathrm{E}-17$ & ADRA1B \\
\hline ENSG00000164050 & 1.092459663 & $1.60 \mathrm{E}-17$ & $1.48 \mathrm{E}-16$ & PLXNB1 \\
\hline ENSG00000232611 & 1.014419347 & $2.24 \mathrm{E}-07$ & $8.55 \mathrm{E}-07$ & AL683813.1 \\
\hline ENSG00000138286 & 1.012503764 & $2.56 \mathrm{E}-17$ & $2.35 \mathrm{E}-16$ & FAM149B1 \\
\hline ENSG00000204852 & 0.586136292 & $2.61 \mathrm{E}-17$ & $2.39 \mathrm{E}-16$ & TCTN1 \\
\hline ENSG00000107968 & 0.672186642 & $2.73 \mathrm{E}-17$ & $2.50 \mathrm{E}-16$ & MAP3К8 \\
\hline ENSG00000103196 & 0.669497228 & $3.21 \mathrm{E}-17$ & $2.93 \mathrm{E}-16$ & CRISPLD2 \\
\hline ENSG00000163694 & 0.664725312 & $3.76 \mathrm{E}-17$ & $3.41 \mathrm{E}-16$ & RBM47 \\
\hline ENSG00000118777 & 0.63684278 & $4.00 \mathrm{E}-17$ & 3.62E-16 & ABCG2 \\
\hline ENSG00000165272 & 0.737450886 & $5.14 \mathrm{E}-17$ & $4.61 \mathrm{E}-16$ & $\mathrm{AQP} 3$ \\
\hline ENSG00000234405 & 1.012989861 & $1.17 \mathrm{E}-05$ & $3.61 \mathrm{E}-05$ & Z69733.1 \\
\hline ENSG00000204282 & 0.999042357 & $2.05 \mathrm{E}-16$ & $1.76 \mathrm{E}-15$ & TNRC6C-AS1 \\
\hline ENSG00000160180 & 1.828562389 & $1.07 \mathrm{E}-16$ & $9.33 \mathrm{E}-16$ & TFF3 \\
\hline ENSG00000091490 & 1.064775 & $1.12 \mathrm{E}-16$ & $9.71 \mathrm{E}-16$ & SEL1L3 \\
\hline ENSG00000174456 & 0.697982291 & $1.56 \mathrm{E}-16$ & $1.35 \mathrm{E}-15$ & C12orf76 \\
\hline ENSG00000224184 & 0.998094957 & $1.45 \mathrm{E}-12$ & $9.33 \mathrm{E}-12$ & MIR3681HG \\
\hline ENSG00000139182 & 0.612905821 & $2.09 \mathrm{E}-16$ & $1.79 \mathrm{E}-15$ & CLSTN3 \\
\hline ENSG00000021762 & 0.664520536 & $2.82 \mathrm{E}-16$ & $2.40 \mathrm{E}-15$ & OSBPL5 \\
\hline ENSG00000024422 & 0.631697665 & $2.90 \mathrm{E}-16$ & $2.47 \mathrm{E}-15$ & EHD2 \\
\hline ENSG00000178209 & 0.59391065 & $4.21 \mathrm{E}-16$ & $3.53 \mathrm{E}-15$ & PLEC \\
\hline ENSG00000213626 & 0.748895824 & $4.77 \mathrm{E}-16$ & $3.99 \mathrm{E}-15$ & LBH \\
\hline ENSG00000203709 & 0.989260567 & $3.38 \mathrm{E}-19$ & $3.53 \mathrm{E}-18$ & Clorf132 \\
\hline ENSG00000120899 & 0.809869697 & $6.04 \mathrm{E}-16$ & $5.03 \mathrm{E}-15$ & PTK2B \\
\hline ENSG00000166171 & 0.634006308 & $6.79 \mathrm{E}-16$ & $5.63 \mathrm{E}-15$ & DPCD \\
\hline ENSG00000119514 & 1.109015536 & $8.36 \mathrm{E}-16$ & $6.87 \mathrm{E}-15$ & GALNT12 \\
\hline ENSG00000166145 & 1.012449601 & $9.25 \mathrm{E}-16$ & $7.55 \mathrm{E}-15$ & SPINT1 \\
\hline ENSG00000139625 & 0.597222522 & $9.56 \mathrm{E}-16$ & $7.80 \mathrm{E}-15$ & МАРЗК 12 \\
\hline ENSG00000130529 & 1.072374016 & $1.03 \mathrm{E}-15$ & $8.41 \mathrm{E}-15$ & TRPM4 \\
\hline ENSG00000089639 & 0.583026836 & $1.04 \mathrm{E}-15$ & $8.48 \mathrm{E}-15$ & GMIP \\
\hline ENSG00000164362 & 0.96421019 & $1.42 \mathrm{E}-15$ & $1.15 \mathrm{E}-14$ & TERT \\
\hline ENSG00000260265 & 0.980352322 & $6.53 \mathrm{E}-06$ & $2.08 \mathrm{E}-05$ & AC1 10760.4 \\
\hline ENSG00000260832 & 0.972908743 & $2.37 \mathrm{E}-05$ & $7.03 \mathrm{E}-05$ & AC125793.1 \\
\hline ENSG00000143224 & 0.603143848 & $1.54 \mathrm{E}-15$ & $1.24 \mathrm{E}-14$ & PPOX \\
\hline ENSG00000166387 & 0.768430513 & $1.83 \mathrm{E}-15$ & $1.47 \mathrm{E}-14$ & PPFIBP2 \\
\hline ENSG00000012124 & 0.751559289 & $2.74 \mathrm{E}-15$ & $2.17 \mathrm{E}-14$ & $\mathrm{CD} 22$ \\
\hline ENSG00000073282 & 0.941931414 & $2.98 \mathrm{E}-15$ & $2.35 \mathrm{E}-14$ & TP63 \\
\hline ENSG00000234424 & 0.96208621 & $1.12 \mathrm{E}-05$ & $3.44 \mathrm{E}-05$ & AL353743.4 \\
\hline ENSG00000162078 & 1.302273759 & $3.84 \mathrm{E}-15$ & $3.01 \mathrm{E}-14$ & ZG16B \\
\hline ENSG00000170190 & 0.646867941 & $5.64 \mathrm{E}-15$ & $4.38 \mathrm{E}-14$ & SLC16A5 \\
\hline ENSG00000277879 & 0.946436382 & $1.64 \mathrm{E}-06$ & $5.64 \mathrm{E}-06$ & AL391988.1 \\
\hline ENSG00000182578 & 0.871074337 & $7.57 \mathrm{E}-15$ & $5.81 \mathrm{E}-14$ & CSF1R \\
\hline ENSG00000204442 & 0.613852863 & $8.56 \mathrm{E}-15$ & $6.55 \mathrm{E}-14$ & FAM155A \\
\hline ENSG00000084764 & 1.238040706 & $9.29 \mathrm{E}-15$ & $7.09 \mathrm{E}-14$ & MAPRE3 \\
\hline ENSG00000187653 & 0.90344409 & $7.66 \mathrm{E}-38$ & $2.02 \mathrm{E}-36$ & TMSB4XP8 \\
\hline ENSG00000125864 & 0.605676615 & $1.40 \mathrm{E}-14$ & $1.06 \mathrm{E}-13$ & BFSP1 \\
\hline ENSG00000004799 & 0.72648243 & $1.43 \mathrm{E}-14$ & $1.07 \mathrm{E}-13$ & PDK4 \\
\hline ENSG00000164690 & 1.021512855 & $1.43 \mathrm{E}-14$ & $1.08 \mathrm{E}-13$ & SHH \\
\hline ENSG00000158006 & 0.675401351 & $1.52 \mathrm{E}-14$ & $1.14 \mathrm{E}-13$ & PAFAH2 \\
\hline ENSG00000102466 & 1.99530516 & $1.53 \mathrm{E}-14$ & $1.14 \mathrm{E}-13$ & FGF14 \\
\hline ENSG00000107902 & 0.928721406 & $2.36 \mathrm{E}-14$ & $1.74 \mathrm{E}-13$ & LHPP \\
\hline ENSG00000163219 & 1.470672943 & $2.40 \mathrm{E}-14$ & $1.77 \mathrm{E}-13$ & ARHGAP25 \\
\hline ENSG00000105327 & 0.659101322 & $2.47 \mathrm{E}-14$ & $1.82 \mathrm{E}-13$ & $\mathrm{BBC} 3$ \\
\hline
\end{tabular}


Table 2 (continued)

\begin{tabular}{|c|c|c|c|c|}
\hline Locus & $\log \mathrm{FC}$ & $P$-Value & FDR & Gene name \\
\hline ENSG00000232931 & 0.879123796 & $1.35 \mathrm{E}-07$ & $5.27 \mathrm{E}-07$ & LINC00342 \\
\hline ENSG00000116885 & 0.944805119 & $2.77 \mathrm{E}-14$ & $2.04 \mathrm{E}-13$ & OSCP1 \\
\hline ENSG00000196542 & 2.000172114 & $3.44 \mathrm{E}-14$ & $2.51 \mathrm{E}-13$ & SPTSSB \\
\hline ENSG00000141469 & 1.257620277 & $4.10 \mathrm{E}-14$ & $2.98 \mathrm{E}-13$ & SLC14A1 \\
\hline ENSG00000207954 & 0.864355996 & $6.57 \mathrm{E}-05$ & 0.0001819 & MIR138-1 \\
\hline ENSG00000269896 & 0.863999249 & $1.98 \mathrm{E}-06$ & $6.72 \mathrm{E}-06$ & AL513477.1 \\
\hline ENSG00000183317 & 1.598192927 & $7.07 \mathrm{E}-14$ & $5.03 \mathrm{E}-13$ & EPHA10 \\
\hline ENSG00000064547 & 1.043247745 & $7.64 \mathrm{E}-14$ & $5.43 \mathrm{E}-13$ & LPAR2 \\
\hline ENSG00000183454 & 1.361311685 & $8.02 \mathrm{E}-14$ & $5.69 \mathrm{E}-13$ & GRIN2A \\
\hline ENSG00000258545 & 0.861885379 & $2.73 \mathrm{E}-05$ & $8.03 \mathrm{E}-05$ & RHOXF1-AS1 \\
\hline ENSG00000171174 & 0.721579865 & $1.15 \mathrm{E}-13$ & $8.07 \mathrm{E}-13$ & RBKS \\
\hline ENSG00000226332 & 0.827459238 & $1.96 \mathrm{E}-10$ & $1.04 \mathrm{E}-09$ & AL354836.1 \\
\hline ENSG00000215769 & 0.819562549 & $6.81 \mathrm{E}-12$ & $4.15 \mathrm{E}-11$ & ARHGAP27P1-BPTFP1-KPNA2P3 \\
\hline ENSG00000069424 & 0.926219371 & $2.49 \mathrm{E}-13$ & $1.70 \mathrm{E}-12$ & KCNAB2 \\
\hline ENSG00000203780 & 1.268825864 & $2.79 \mathrm{E}-13$ & $1.90 \mathrm{E}-12$ & FANK1 \\
\hline ENSG00000147642 & 0.674353182 & $3.40 \mathrm{E}-13$ & $2.30 \mathrm{E}-12$ & SYBU \\
\hline ENSG00000261786 & 0.80298847 & $9.74 \mathrm{E}-14$ & $6.87 \mathrm{E}-13$ & AC006058.1 \\
\hline ENSG00000224086 & 0.750479112 & $5.45 \mathrm{E}-07$ & $1.98 \mathrm{E}-06$ & AC245452.1 \\
\hline ENSG00000251562 & 0.749923658 & $6.90 \mathrm{E}-14$ & $4.92 \mathrm{E}-13$ & MALAT1 \\
\hline ENSG00000108821 & 0.721390767 & $5.89 \mathrm{E}-13$ & $3.92 \mathrm{E}-12$ & COL1A1 \\
\hline ENSG00000163993 & 0.950679444 & $6.17 \mathrm{E}-13$ & $4.10 \mathrm{E}-12$ & S100P \\
\hline ENSG00000119686 & 1.315494173 & $8.81 \mathrm{E}-13$ & $5.80 \mathrm{E}-12$ & FLVCR2 \\
\hline ENSG00000267481 & 0.748507005 & 0.00019344 & 0.000499816 & AC011477.2 \\
\hline ENSG00000161677 & 0.65441487 & $8.86 \mathrm{E}-13$ & $5.82 \mathrm{E}-12$ & JOSD2 \\
\hline ENSG00000168477 & 1.714597499 & $9.18 \mathrm{E}-13$ & $6.02 \mathrm{E}-12$ & TNXB \\
\hline ENSG00000167535 & 0.66107174 & $1.08 \mathrm{E}-12$ & $7.02 \mathrm{E}-12$ & CACNB3 \\
\hline ENSG00000188177 & 1.190091354 & $1.32 \mathrm{E}-12$ & $8.55 \mathrm{E}-12$ & ZC3H6 \\
\hline ENSG00000277778 & 0.730989212 & $3.18 \mathrm{E}-07$ & $1.19 \mathrm{E}-06$ & PGM5P2 \\
\hline ENSG00000099889 & 0.874014164 & $2.18 \mathrm{E}-12$ & $1.39 \mathrm{E}-11$ & ARVCF \\
\hline ENSG00000257315 & 0.726622407 & $2.48 \mathrm{E}-12$ & $1.57 \mathrm{E}-11$ & ZBED6 \\
\hline ENSG00000161395 & 0.681576338 & $2.54 \mathrm{E}-12$ & $1.61 \mathrm{E}-11$ & PGAP3 \\
\hline ENSG00000283646 & 0.715148035 & $5.47 \mathrm{E}-13$ & $3.66 \mathrm{E}-12$ & LINC02009 \\
\hline ENSG00000274307 & 0.705188548 & 0.000339574 & 0.000836261 & AC023449.2 \\
\hline ENSG00000167103 & 1.332705616 & $4.92 \mathrm{E}-12$ & $3.05 \mathrm{E}-11$ & PIP5KL1 \\
\hline ENSG00000159423 & 0.611704098 & $6.38 \mathrm{E}-12$ & $3.90 \mathrm{E}-11$ & ALDH4A1 \\
\hline ENSG00000278897 & 0.70098086 & 0.000273415 & 0.000687648 & AC020951.1 \\
\hline ENSG00000162426 & 1.314896899 & $8.38 \mathrm{E}-12$ & $5.07 \mathrm{E}-11$ & SLC45A1 \\
\hline ENSG00000188064 & 0.633873994 & $1.16 \mathrm{E}-11$ & $6.95 \mathrm{E}-11$ & WNT7B \\
\hline ENSG00000187952 & 0.691227099 & 0.000538949 & 0.001287183 & HS6ST1P1 \\
\hline ENSG00000108641 & 0.676095788 & $1.31 \mathrm{E}-11$ & $7.82 \mathrm{E}-11$ & B9D1 \\
\hline ENSG00000139178 & 0.828600996 & $1.34 \mathrm{E}-11$ & $7.98 \mathrm{E}-11$ & C1RL \\
\hline ENSG00000253669 & 0.690702691 & $6.08 \mathrm{E}-09$ & $2.78 \mathrm{E}-08$ & AP003356.1 \\
\hline ENSG00000281881 & 0.679695644 & $1.99 \mathrm{E}-05$ & $5.96 \mathrm{E}-05$ & SPRY4-IT1 \\
\hline ENSG00000176371 & 0.682561022 & $3.05 \mathrm{E}-11$ & $1.75 \mathrm{E}-10$ & ZSCAN2 \\
\hline ENSG00000066248 & 0.600563626 & $3.22 \mathrm{E}-11$ & $1.85 \mathrm{E}-10$ & NGEF \\
\hline ENSG00000239713 & 0.790898223 & $3.30 \mathrm{E}-11$ & $1.89 \mathrm{E}-10$ & APOBEC3G \\
\hline ENSG00000084710 & 0.814302414 & $4.97 \mathrm{E}-11$ & $2.82 \mathrm{E}-10$ & EFR3B \\
\hline ENSG00000213315 & 0.674927745 & 0.001017341 & 0.002306342 & AL122020.1 \\
\hline ENSG00000233895 & 0.671323329 & $2.93 \mathrm{E}-05$ & $8.55 \mathrm{E}-05$ & AL121761.1 \\
\hline ENSG00000205593 & 1.297364282 & $7.30 \mathrm{E}-11$ & $4.05 \mathrm{E}-10$ & DENND6B \\
\hline ENSG00000173156 & 0.660509744 & $7.44 \mathrm{E}-11$ & $4.13 \mathrm{E}-10$ & RHOD \\
\hline ENSG00000129757 & 1.280060934 & $8.31 \mathrm{E}-11$ & $4.60 \mathrm{E}-10$ & CDKN1C \\
\hline ENSG00000165929 & 1.580465307 & $1.11 \mathrm{E}-10$ & $6.07 \mathrm{E}-10$ & $\mathrm{TC} 2 \mathrm{~N}$ \\
\hline ENSG00000111319 & 0.916384563 & $1.54 \mathrm{E}-10$ & $8.30 \mathrm{E}-10$ & SCNN1A \\
\hline ENSG00000226137 & 0.662532844 & $2.08 \mathrm{E}-17$ & $1.92 \mathrm{E}-16$ & BAIAP2-AS1 \\
\hline ENSG00000131779 & 0.645897178 & $1.88 \mathrm{E}-10$ & $1.00 \mathrm{E}-09$ & PEX11B \\
\hline ENSG00000149972 & 1.274126912 & $1.95 \mathrm{E}-10$ & $1.04 \mathrm{E}-09$ & CNTN5 \\
\hline ENSG00000250072 & 0.661140207 & $3.69 \mathrm{E}-05$ & 0.000106104 & AC091940.1 \\
\hline ENSG00000158458 & 0.771645473 & $2.17 \mathrm{E}-10$ & $1.15 \mathrm{E}-09$ & NRG2 \\
\hline ENSG00000171443 & 0.620783725 & $2.33 \mathrm{E}-10$ & $1.23 \mathrm{E}-09$ & ZNF524 \\
\hline ENSG00000233452 & 0.657023126 & 0.001884719 & 0.004066423 & STXBP5-AS1 \\
\hline ENSG00000146966 & 0.580752824 & $3.35 \mathrm{E}-10$ & $1.74 \mathrm{E}-09$ & DENND2A \\
\hline ENSG00000245849 & 0.649770435 & 0.001938789 & 0.004172798 & RAD51-AS1 \\
\hline ENSG00000220785 & 0.643682307 & 0.000249588 & 0.000632447 & MTMR9LP \\
\hline ENSG00000160703 & 0.613565082 & $3.93 \mathrm{E}-10$ & $2.02 \mathrm{E}-09$ & NLRX1 \\
\hline ENSG00000006756 & 0.786505348 & $4.19 \mathrm{E}-10$ & $2.15 \mathrm{E}-09$ & ARSD \\
\hline
\end{tabular}


Table 2 (continued)

\begin{tabular}{|c|c|c|c|c|}
\hline Locus & $\log \mathrm{FC}$ & $P$-Value & FDR & Gene name \\
\hline ENSG00000106078 & 1.171724535 & $4.43 \mathrm{E}-10$ & $2.27 \mathrm{E}-09$ & COBL \\
\hline ENSG00000169248 & 0.790213883 & $5.21 \mathrm{E}-10$ & $2.65 \mathrm{E}-09$ & CXCL11 \\
\hline ENSG00000143127 & 0.858887046 & $5.91 \mathrm{E}-10$ & $2.98 \mathrm{E}-09$ & ITGA10 \\
\hline ENSG00000230487 & 0.635330515 & $7.29 \mathrm{E}-05$ & 0.000200601 & PSMG3-AS1 \\
\hline ENSG00000169231 & 0.71986648 & $6.66 \mathrm{E}-10$ & $3.36 \mathrm{E}-09$ & THBS3 \\
\hline ENSG00000264112 & 0.630435915 & $3.88 \mathrm{E}-13$ & $2.61 \mathrm{E}-12$ & AC015813.1 \\
\hline ENSG00000272512 & 0.629649183 & 0.00016484 & 0.000430322 & AL645608.8 \\
\hline ENSG00000248008 & 0.626222703 & $1.44 \mathrm{E}-15$ & $1.16 \mathrm{E}-14$ & NRAV \\
\hline ENSG00000165698 & 1.042720757 & $1.01 \mathrm{E}-09$ & $4.98 \mathrm{E}-09$ & SPACA9 \\
\hline ENSG00000167065 & 0.691874401 & $1.17 \mathrm{E}-09$ & $5.78 \mathrm{E}-09$ & DUSP18 \\
\hline ENSG00000283160 & 0.62478041 & 0.003658265 & 0.007489349 & MIR4521 \\
\hline ENSG00000167306 & 0.684016305 & $1.74 \mathrm{E}-09$ & $8.41 \mathrm{E}-09$ & MYO5B \\
\hline ENSG00000187987 & 1.030707676 & $1.80 \mathrm{E}-09$ & $8.67 \mathrm{E}-09$ & ZSCAN23 \\
\hline ENSG00000185386 & 0.750119972 & $1.89 \mathrm{E}-09$ & $9.10 \mathrm{E}-09$ & MAPK11 \\
\hline ENSG00000281026 & 0.623674242 & 0.00174105 & 0.003777549 & N4BP2L2-IT2 \\
\hline ENSG00000167895 & 1.301863777 & $1.94 \mathrm{E}-09$ & $9.32 \mathrm{E}-09$ & TMC8 \\
\hline ENSG00000270640 & 0.621162265 & 0.003395331 & 0.006999073 & AC104695.3 \\
\hline ENSG00000263155 & 1.349972416 & $2.25 \mathrm{E}-09$ & $1.07 \mathrm{E}-08$ & MYZAP \\
\hline ENSG00000125637 & 0.809041824 & $2.30 \mathrm{E}-09$ & $1.10 \mathrm{E}-08$ & PSD4 \\
\hline ENSG00000178531 & 0.6403658 & $2.47 \mathrm{E}-09$ & $1.18 \mathrm{E}-08$ & CTXN1 \\
\hline ENSG00000006210 & 1.386770722 & 3.03E-09 & $1.43 \mathrm{E}-08$ & CX3CL1 \\
\hline ENSG00000196476 & 0.703193796 & $3.10 \mathrm{E}-09$ & $1.46 \mathrm{E}-08$ & C20orf96 \\
\hline ENSG00000114841 & 0.724964893 & $3.49 \mathrm{E}-09$ & $1.63 \mathrm{E}-08$ & DNAH1 \\
\hline ENSG00000248690 & 0.616225449 & 0.005847093 & 0.011483426 & HAS2-AS1 \\
\hline ENSG00000128849 & 0.993028568 & $3.72 \mathrm{E}-09$ & $1.74 \mathrm{E}-08$ & CGNL1 \\
\hline ENSG00000250903 & 0.616173957 & $7.24 \mathrm{E}-15$ & $5.56 \mathrm{E}-14$ & GMDS-AS1 \\
\hline ENSG00000139192 & 0.677957048 & $4.54 \mathrm{E}-09$ & $2.10 \mathrm{E}-08$ & TAPBPL \\
\hline ENSG00000273888 & 0.615241762 & 0.000253027 & 0.00064005 & FRMD6-AS1 \\
\hline ENSG00000276116 & 0.614111505 & $8.00 \mathrm{E}-05$ & 0.000218844 & FUT8-AS1 \\
\hline ENSG00000078114 & 0.779192836 & $5.73 \mathrm{E}-09$ & $2.63 \mathrm{E}-08$ & NEBL \\
\hline ENSG00000223745 & 0.609457977 & $6.90 \mathrm{E}-08$ & $2.79 \mathrm{E}-07$ & CCDC18-AS1 \\
\hline ENSG00000276476 & 0.595189913 & $3.36 \mathrm{E}-05$ & $9.71 \mathrm{E}-05$ & LINC00540 \\
\hline ENSG00000104332 & 1.024730115 & $6.35 \mathrm{E}-09$ & $2.90 \mathrm{E}-08$ & SFRP1 \\
\hline ENSG00000187688 & 0.74390243 & $6.40 \mathrm{E}-09$ & $2.92 \mathrm{E}-08$ & TRPV2 \\
\hline ENSG00000168961 & 1.249308012 & $7.43 \mathrm{E}-09$ & $3.37 \mathrm{E}-08$ & LGALS9 \\
\hline ENSG00000181444 & 1.289743152 & $7.97 \mathrm{E}-09$ & $3.60 \mathrm{E}-08$ & ZNF467 \\
\hline ENSG00000007516 & 0.7190471 & $9.06 \mathrm{E}-09$ & $4.06 \mathrm{E}-08$ & BAIAP3 \\
\hline ENSG00000074964 & 0.723682951 & $9.90 \mathrm{E}-09$ & $4.42 \mathrm{E}-08$ & ARHGEF10L \\
\hline ENSG00000153233 & 0.860394698 & $1.06 \mathrm{E}-08$ & $4.73 \mathrm{E}-08$ & PTPRR \\
\hline ENSG00000158125 & 0.749227047 & $1.34 \mathrm{E}-08$ & $5.87 \mathrm{E}-08$ & XDH \\
\hline ENSG00000105982 & 0.809043212 & $1.50 \mathrm{E}-08$ & $6.57 \mathrm{E}-08$ & RNF32 \\
\hline ENSG00000130751 & 0.996208061 & $2.12 \mathrm{E}-08$ & $9.07 \mathrm{E}-08$ & NPAS1 \\
\hline ENSG00000187583 & 0.628689467 & $2.15 \mathrm{E}-08$ & $9.21 \mathrm{E}-08$ & PLEKHN1 \\
\hline ENSG00000129295 & 0.804174666 & $3.78 \mathrm{E}-08$ & $1.58 \mathrm{E}-07$ & LRRC6 \\
\hline ENSG00000136167 & 0.72905745 & 4.42E-08 & $1.83 \mathrm{E}-07$ & LCP1 \\
\hline ENSG00000109107 & 0.624763894 & $4.83 \mathrm{E}-08$ & $1.99 \mathrm{E}-07$ & ALDOC \\
\hline ENSG00000102796 & 0.580283788 & $4.92 \mathrm{E}-08$ & $2.02 \mathrm{E}-07$ & DHRS12 \\
\hline ENSG00000169926 & 0.738403193 & $5.42 \mathrm{E}-08$ & $2.22 \mathrm{E}-07$ & KLF13 \\
\hline ENSG00000169583 & 0.867293251 & $6.71 \mathrm{E}-08$ & $2.72 \mathrm{E}-07$ & CLIC3 \\
\hline ENSG00000105204 & 0.693379327 & $6.79 \mathrm{E}-08$ & $2.75 \mathrm{E}-07$ & DYRK1B \\
\hline ENSG00000089127 & 0.795662065 & $8.09 \mathrm{E}-08$ & $3.24 \mathrm{E}-07$ & OAS1 \\
\hline ENSG00000169220 & 0.610978587 & $8.83 \mathrm{E}-08$ & $3.53 \mathrm{E}-07$ & RGS14 \\
\hline ENSG00000160190 & 0.656350516 & $9.89 \mathrm{E}-08$ & $3.94 \mathrm{E}-07$ & SLC37A1 \\
\hline ENSG00000156218 & 0.750817532 & $1.07 \mathrm{E}-07$ & $4.25 \mathrm{E}-07$ & ADAMTSL3 \\
\hline ENSG00000105854 & 0.64320919 & $1.18 \mathrm{E}-07$ & $4.64 \mathrm{E}-07$ & PON2 \\
\hline ENSG00000103034 & 0.978032576 & $1.35 \mathrm{E}-07$ & $5.28 \mathrm{E}-07$ & NDRG4 \\
\hline ENSG00000008517 & 0.607365229 & $1.35 \mathrm{E}-07$ & $5.29 \mathrm{E}-07$ & IL32 \\
\hline ENSG00000163617 & 0.881935404 & $1.36 \mathrm{E}-07$ & $5.33 \mathrm{E}-07$ & CCDC191 \\
\hline ENSG00000184194 & 0.756086964 & $1.44 \mathrm{E}-07$ & $5.61 \mathrm{E}-07$ & GPR173 \\
\hline ENSG00000124116 & 0.704374738 & $1.47 \mathrm{E}-07$ & $5.72 \mathrm{E}-07$ & WFDC3 \\
\hline ENSG00000197279 & 0.61212415 & $1.48 \mathrm{E}-07$ & $5.74 \mathrm{E}-07$ & ZNF165 \\
\hline ENSG00000155265 & 1.06380425 & $1.92 \mathrm{E}-07$ & $7.39 \mathrm{E}-07$ & GOLGA7B \\
\hline ENSG00000196420 & 0.668634419 & $2.03 \mathrm{E}-07$ & $7.76 \mathrm{E}-07$ & S100A5 \\
\hline ENSG00000163995 & 0.914334457 & $2.21 \mathrm{E}-07$ & $8.44 \mathrm{E}-07$ & ABLIM2 \\
\hline ENSG00000173267 & 0.875959067 & $2.32 \mathrm{E}-07$ & $8.85 \mathrm{E}-07$ & SNCG \\
\hline ENSG00000174951 & 0.583579922 & $2.48 \mathrm{E}-07$ & $9.40 \mathrm{E}-07$ & FUT1 \\
\hline
\end{tabular}


Table 2 (continued)

\begin{tabular}{|c|c|c|c|c|}
\hline Locus & $\log \mathrm{FC}$ & $P$-Value & FDR & Gene name \\
\hline ENSG00000166578 & 0.59325713 & $2.97 \mathrm{E}-07$ & $1.12 \mathrm{E}-06$ & IQCD \\
\hline ENSG00000134030 & 0.721745796 & $3.68 \mathrm{E}-07$ & $1.36 \mathrm{E}-06$ & CTIF \\
\hline ENSG00000133640 & 0.867286671 & $3.91 \mathrm{E}-07$ & $1.45 \mathrm{E}-06$ & LRRIQ1 \\
\hline ENSG00000262576 & 0.845341331 & $4.37 \mathrm{E}-07$ & $1.60 \mathrm{E}-06$ & PCDHGA4 \\
\hline ENSG00000160781 & 0.937385076 & $4.56 \mathrm{E}-07$ & $1.67 \mathrm{E}-06$ & PAQR6 \\
\hline ENSG00000050165 & 0.701579384 & $5.28 \mathrm{E}-07$ & $1.92 \mathrm{E}-06$ & DKK3 \\
\hline ENSG00000111863 & 0.721347277 & $6.34 \mathrm{E}-07$ & $2.29 \mathrm{E}-06$ & ADTRP \\
\hline ENSG00000160325 & 0.946677553 & $6.89 \mathrm{E}-07$ & $2.48 \mathrm{E}-06$ & CACFD1 \\
\hline ENSG00000198846 & 0.743926359 & $7.19 \mathrm{E}-07$ & $2.58 \mathrm{E}-06$ & TOX \\
\hline ENSG00000166262 & 1.011056155 & $7.77 \mathrm{E}-07$ & $2.77 \mathrm{E}-06$ & FAM227B \\
\hline ENSG00000239282 & 0.740685304 & $9.03 \mathrm{E}-07$ & $3.20 \mathrm{E}-06$ & CASTOR1 \\
\hline ENSG00000122547 & 0.589219386 & $1.04 \mathrm{E}-06$ & $3.67 \mathrm{E}-06$ & EEPD1 \\
\hline ENSG00000115556 & 0.759404536 & $1.29 \mathrm{E}-06$ & $4.50 \mathrm{E}-06$ & PLCD4 \\
\hline ENSG00000146021 & 0.996087722 & $1.42 \mathrm{E}-06$ & $4.94 \mathrm{E}-06$ & KLHL3 \\
\hline ENSG00000167554 & 0.801922519 & $1.58 \mathrm{E}-06$ & $5.45 \mathrm{E}-06$ & ZNF610 \\
\hline ENSG00000159899 & 0.607690901 & $1.59 \mathrm{E}-06$ & $5.47 \mathrm{E}-06$ & NPR2 \\
\hline ENSG00000085831 & 0.88967755 & $1.86 \mathrm{E}-06$ & $6.33 \mathrm{E}-06$ & TTC39A \\
\hline ENSG00000167600 & 0.949538739 & $1.95 \mathrm{E}-06$ & $6.64 \mathrm{E}-06$ & CYP2S1 \\
\hline ENSG00000135298 & 0.65393669 & $2.08 \mathrm{E}-06$ & $7.03 \mathrm{E}-06$ & ADGRB3 \\
\hline ENSG00000185345 & 1.087332962 & $2.19 \mathrm{E}-06$ & $7.38 \mathrm{E}-06$ & PRKN \\
\hline ENSG00000079974 & 0.704729252 & $2.33 \mathrm{E}-06$ & $7.83 \mathrm{E}-06$ & RABL2B \\
\hline ENSG00000213085 & 0.908492786 & $2.67 \mathrm{E}-06$ & $8.89 \mathrm{E}-06$ & CFAP45 \\
\hline ENSG00000137261 & 1.001309402 & $4.72 \mathrm{E}-06$ & $1.53 \mathrm{E}-05$ & KIAA0319 \\
\hline ENSG00000131620 & 0.893864552 & $5.41 \mathrm{E}-06$ & $1.74 \mathrm{E}-05$ & ANO1 \\
\hline ENSG00000005379 & 0.634150616 & $5.44 \mathrm{E}-06$ & $1.75 \mathrm{E}-05$ & TSPOAP1 \\
\hline ENSG00000170927 & 1.061916014 & $5.77 \mathrm{E}-06$ & $1.85 \mathrm{E}-05$ & PKHD1 \\
\hline ENSG00000183638 & 0.95696744 & $5.87 \mathrm{E}-06$ & $1.88 \mathrm{E}-05$ & RP1L1 \\
\hline ENSG00000185634 & 0.655532658 & $6.41 \mathrm{E}-06$ & $2.04 \mathrm{E}-05$ & SHC4 \\
\hline ENSG00000178026 & 0.704425401 & $7.44 \mathrm{E}-06$ & $2.35 \mathrm{E}-05$ & LRRC75B \\
\hline ENSG00000007237 & 0.94018185 & $7.53 \mathrm{E}-06$ & $2.38 \mathrm{E}-05$ & GAS7 \\
\hline ENSG00000063438 & 0.850087695 & $7.99 \mathrm{E}-06$ & $2.52 \mathrm{E}-05$ & AHRR \\
\hline ENSG00000078081 & 0.943742842 & $1.00 \mathrm{E}-05$ & $3.13 \mathrm{E}-05$ & LAMP3 \\
\hline ENSG00000152779 & 0.893034503 & $1.16 \mathrm{E}-05$ & $3.58 \mathrm{E}-05$ & SLC16A12 \\
\hline ENSG00000105649 & 0.629673856 & $1.17 \mathrm{E}-05$ & $3.59 \mathrm{E}-05$ & RAB3A \\
\hline ENSG00000101670 & 0.641918014 & $1.74 \mathrm{E}-05$ & $5.23 \mathrm{E}-05$ & LIPG \\
\hline ENSG00000153246 & 0.640475 & $2.34 \mathrm{E}-05$ & $6.95 \mathrm{E}-05$ & PLA2R1 \\
\hline ENSG00000197013 & 0.895543676 & $2.42 \mathrm{E}-05$ & $7.15 \mathrm{E}-05$ & ZNF429 \\
\hline ENSG00000100027 & 0.703475726 & $2.55 \mathrm{E}-05$ & $7.54 \mathrm{E}-05$ & YPEL1 \\
\hline ENSG00000222009 & 1.003174533 & $2.78 \mathrm{E}-05$ & $8.16 \mathrm{E}-05$ & BTBD19 \\
\hline ENSG00000077092 & 0.583904079 & $2.82 \mathrm{E}-05$ & $8.25 \mathrm{E}-05$ & RARB \\
\hline ENSG00000138271 & 0.879042367 & $2.88 \mathrm{E}-05$ & $8.41 \mathrm{E}-05$ & GPR87 \\
\hline ENSG00000215788 & 0.630842347 & $4.98 \mathrm{E}-05$ & 0.000140178 & TNFRSF25 \\
\hline ENSG00000111907 & 0.610094964 & $5.19 \mathrm{E}-05$ & 0.000145846 & TPD52L1 \\
\hline ENSG00000148225 & 0.603765317 & $5.24 \mathrm{E}-05$ & 0.000146979 & WDR31 \\
\hline ENSG00000265190 & 0.796463642 & $5.25 \mathrm{E}-05$ & 0.00014743 & ANXA8 \\
\hline ENSG00000166780 & 0.609717829 & $5.44 \mathrm{E}-05$ & 0.00015227 & C16orf45 \\
\hline ENSG00000196917 & 0.687020342 & $5.62 \mathrm{E}-05$ & 0.000157013 & HCAR1 \\
\hline ENSG00000243749 & 0.682870409 & $6.23 \mathrm{E}-05$ & 0.00017307 & TMEM35B \\
\hline ENSG00000100100 & 0.614989243 & $6.46 \mathrm{E}-05$ & 0.000179064 & PIK3IP1 \\
\hline ENSG00000185261 & 0.808099048 & $6.60 \mathrm{E}-05$ & 0.000182782 & KIAA0825 \\
\hline ENSG00000176714 & 0.719708594 & $6.65 \mathrm{E}-05$ & 0.000183929 & $\mathrm{CCDC} 121$ \\
\hline ENSG00000189350 & 0.682674219 & $8.27 \mathrm{E}-05$ & 0.00022538 & TOGARAM2 \\
\hline ENSG00000117586 & 0.66232365 & $9.60 \mathrm{E}-05$ & 0.000259115 & TNFSF4 \\
\hline ENSG00000163283 & 0.702973737 & $9.69 \mathrm{E}-05$ & 0.000261336 & ALPP \\
\hline ENSG00000100628 & 0.840396776 & 0.000102783 & 0.000275833 & ASB2 \\
\hline ENSG00000204936 & 0.799723751 & 0.000132176 & 0.000349991 & CD177 \\
\hline ENSG00000249242 & 0.674349922 & 0.00013392 & 0.000354394 & TMEM150C \\
\hline ENSG00000154589 & 0.621650835 & 0.000154203 & 0.000405048 & LY96 \\
\hline ENSG00000137103 & 0.813157861 & 0.000158856 & 0.000416188 & TMEM8B \\
\hline ENSG00000186417 & 0.845874331 & 0.000174472 & 0.000454112 & GLDN \\
\hline ENSG00000168702 & 0.829015848 & 0.000183578 & 0.000476395 & LRP1B \\
\hline ENSG00000177694 & 0.678072111 & 0.000201099 & 0.000518177 & NAALADL2 \\
\hline ENSG00000137460 & 0.621505207 & 0.000215133 & 0.000551848 & FHDC1 \\
\hline ENSG00000233493 & 0.606768387 & 0.000304964 & 0.000759001 & TMEM238 \\
\hline ENSG00000135525 & 0.592393732 & 0.000307346 & 0.00076377 & MAP7 \\
\hline ENSG00000177076 & 0.595537696 & 0.000354308 & 0.000869936 & ACER2 \\
\hline
\end{tabular}


Table 2 (continued)

\begin{tabular}{|c|c|c|c|c|}
\hline Locus & $\log \mathrm{FC}$ & $P$-Value & FDR & Gene name \\
\hline ENSG00000172456 & 0.624018639 & 0.000361318 & 0.000885657 & FGGY \\
\hline ENSG00000112303 & 0.791611125 & 0.000395261 & 0.00096382 & VNN2 \\
\hline ENSG00000174327 & 0.660320887 & 0.000409421 & 0.000994655 & SLC16A13 \\
\hline ENSG00000118997 & 0.801710893 & 0.000462226 & 0.001115511 & DNAH7 \\
\hline ENSG00000255690 & 0.769175757 & 0.000506713 & 0.001215944 & TRIL \\
\hline ENSG00000010295 & 0.685112629 & 0.000525958 & 0.001259364 & IFFO1 \\
\hline ENSG00000145107 & 0.585678306 & 0.000567754 & 0.001349109 & TM4SF19 \\
\hline ENSG00000168026 & 0.683159923 & 0.000690836 & 0.001616374 & TTC21A \\
\hline ENSG00000137285 & 0.737035799 & 0.000813426 & 0.00187585 & TUBB2B \\
\hline ENSG00000156510 & 0.685659195 & 0.000879493 & 0.002015241 & HKDC1 \\
\hline ENSG00000153237 & 0.729462631 & 0.000921302 & 0.002105764 & $\mathrm{CCDC} 148$ \\
\hline ENSG00000131849 & 0.669939869 & 0.001076571 & 0.002430196 & ZNF132 \\
\hline ENSG00000169550 & 0.724738148 & 0.0011291 & 0.002537865 & MUC15 \\
\hline ENSG00000253649 & 0.611969408 & 0.001576863 & 0.003452116 & PRSS51 \\
\hline ENSG00000264230 & 0.647657643 & 0.001653031 & 0.003600257 & ANXA8L1 \\
\hline ENSG00000183401 & 0.685190181 & 0.002421385 & 0.005126606 & CCDC159 \\
\hline ENSG00000166816 & 0.611266978 & 0.00242422 & 0.005130953 & LDHD \\
\hline ENSG00000108932 & 0.615640297 & 0.002572756 & 0.00541913 & SLC16A6 \\
\hline ENSG00000106125 & 0.593529032 & 0.003116381 & 0.006461581 & MINDY4 \\
\hline ENSG00000183091 & 0.60347346 & 0.003149988 & 0.006521995 & NEB \\
\hline ENSG00000152582 & 0.655526014 & 0.004260382 & 0.008617234 & SPEF2 \\
\hline
\end{tabular}

polyethylenimine polymer $(1 \mathrm{mg} / \mathrm{ml})$ (Polysciences Inc; 23966-1), which had been preincubated for $15 \mathrm{~min}$ at room temperature and processed as explained above. Viral particles were concentrated using Lenti-X Concentrator product (Clontech; 631232) and used to infect MDA-MB-231 cells. For SUV-39H1-GFP rescue experiments, MDA-MB-231 cells infected with either shCT or shLOXL2 were selected for $48 \mathrm{~h}$ with puromycin (Puro) $(2.5 \mu \mathrm{g} / \mathrm{ml})$. After selection, cells were seeded for $24 \mathrm{~h}$ and transfected with CGApCAGGS-SUV39H1-EGFP-IRES-Puro vector $(2.5 \mu \mathrm{g})$ using TransIT-X2 Dynamic Delivery System (Mirus Bio LLC; MIR600Q).

\section{Antibodies and other reagents}

The following antibodies were used: anti-FLAG (F7425, Sigma), anti-LOXL2 (NP1-32954, Novus), anti-H3K4me3 (07-473, EMD Millipore), anti-H3K9me3 (07-442, Millipore), anti-phospho-histone H2AX (S139) clone JBW301 (05-636, EMD Millipore), anti-GFP (ab6556, Abcam), anti-53BP1 (NB100-904, Novus Biol.), anti-phosphoCHK1 (S317) (A300-163A, Bethyl), anti-CHK2 clone 7 (05-649, EMD Millipore), anti-cleaved caspase 3 (Asp175) (9661, Cell Signaling), anti-KAP-1 (ab10484, Abcam), anti-Phospho-KAP-1 (S824) (A300-767A, Bethyl), antitubulin (T9026, Sigma), anti-phospho-H3 (S10) (06-570, Millipore), and anti-histone H3 (ab1791, Abcam). The following chemical reagents were used: doxorubicin hydrochloride (Sigma; D1515), cordycepin (Sigma; C3394), and the ATM inhibitor KU55933 (Sigma; SML1109). An anti-H3K4ox antibody was generated from a synthetic peptide in which fluorenylmethoxycarbonyl protecting group (Fmoc)-6-hydroxynorleucine (BAA1117, Iris Biotech) rather than Fmoc-lysine(Boc) was incorporated at position 4 . The peptide was purified $(>95 \%)$ by reversedphase high-performance liquid chromatography and its identity was confirmed by mass spectrometry, after which it was coupled to keyhole limpet hemocyanin $(\mathrm{KLH})$ for antibody production. Antibody information can be found in the Supplementary Table 1.

\section{Cell extracts, histone isolation, and PDX extraction}

To obtain nuclear fractions of LOXL2-Flag-transfected MCF-7 cells and HEK293T cells, cells were first lysed in soft-lysis buffer ( $50 \mathrm{mM}$ Tris, $2 \mathrm{mM}$ EDTA, $0.1 \%$ NP-40, and $10 \%$ glycerol, supplemented with protease and phosphatase inhibitors) for $5 \mathrm{~min}$ on ice. Samples were centrifuged at $900 \times g$ for $15 \mathrm{~min}$, and the supernatant was discarded. The nuclear pellet was lysed in high-salt lysis buffer $(20 \mathrm{mM}$ HEPES pH 7.4, $350 \mathrm{mM} \mathrm{NaCl}, 1 \mathrm{mM}$ $\mathrm{MgCl}_{2}, 0.5 \%$ Triton $\mathrm{X}-100$, and $10 \%$ glycerol, supplemented with protease and phosphatase inhibitors) for $30 \mathrm{~min}$ at $4{ }^{\circ} \mathrm{C}$, and samples were centrifuged at $16,000 \times g$ for 10 min supernatant $\mathrm{NaCl}$ concentration was reduced to $300 \mathrm{mM} \mathrm{NaCl}$ by adding balance buffer ( $20 \mathrm{mM}$ HEPES $\mathrm{pH}$ $7.4,1 \mathrm{mM} \mathrm{MgCl}_{2}$, and $10 \mathrm{mM} \mathrm{KCl}$ ). Cells extracts from the different breast cancer cell lines were obtained with SDS lysis buffer (2\% SDS, $50 \mathrm{mM}$ Tris- $\mathrm{HCl}$, and $10 \%$ glycerol). Histones were isolated from the different breast cancer cell lines using acid precipitation. For this, cell monolayers were first scraped in $1 \mathrm{ml}$ of lysis buffer $(10 \mathrm{mM}$ Tris $\mathrm{pH} 6.5$, 
Table 3 Genes downregulated in LOXL2 knockdown

\begin{tabular}{|c|c|c|c|c|}
\hline Locus & $\log \mathrm{FC}$ & $P$-Value & FDR & Gene name \\
\hline ENSG00000134013 & -3.053434126 & 0 & 0 & LOXL2 \\
\hline ENSG00000122545 & -1.493781116 & $6.6 \mathrm{E}-272$ & $1.45 \mathrm{E}-268$ & SEP-T7 \\
\hline ENSG00000137801 & -1.65563595 & $1.11 \mathrm{E}-262$ & $2.08 \mathrm{E}-259$ & THBS1 \\
\hline ENSG00000112062 & -1.367605967 & $1.43 \mathrm{E}-252$ & $2.34 \mathrm{E}-249$ & MAPK14 \\
\hline ENSG00000146281 & -1.644281336 & $6.67 \mathrm{E}-244$ & $9.73 \mathrm{E}-241$ & PM20D2 \\
\hline ENSG00000105971 & -1.358844865 & $4.29 \mathrm{E}-189$ & $3.31 \mathrm{E}-186$ & CAV2 \\
\hline ENSG00000117500 & -1.227263597 & $2.54 \mathrm{E}-176$ & $1.67 \mathrm{E}-173$ & TMED5 \\
\hline ENSG00000172380 & -1.135404374 & $1.81 \mathrm{E}-162$ & $1.08 \mathrm{E}-159$ & GNG12 \\
\hline ENSG00000152558 & -1.10232016 & $1.28 \mathrm{E}-160$ & $7.33 \mathrm{E}-158$ & TMEM123 \\
\hline ENSG00000100462 & -1.134694357 & $2.31 \mathrm{E}-160$ & $1.26 \mathrm{E}-157$ & PRMT5 \\
\hline ENSG00000213281 & -1.098589526 & $6.65 \mathrm{E}-160$ & $3.49 \mathrm{E}-157$ & NRAS \\
\hline ENSG00000162521 & -1.195180927 & $7.41 \mathrm{E}-138$ & $2.95 \mathrm{E}-135$ & RBBP4 \\
\hline ENSG00000196396 & -1.038088551 & $1.5 \mathrm{E}-136$ & $5.64 \mathrm{E}-134$ & PTPN1 \\
\hline ENSG00000182400 & -1.288279943 & $9.25 \mathrm{E}-134$ & $3.2 \mathrm{E}-131$ & TRAPPC6B \\
\hline ENSG00000115339 & -1.421874178 & $1.01 \mathrm{E}-132$ & $3.31 \mathrm{E}-130$ & GALNT3 \\
\hline ENSG00000105810 & -1.03878264 & $1.67 \mathrm{E}-132$ & $5.35 \mathrm{E}-130$ & CDK6 \\
\hline ENSG00000105849 & -1.177181646 & $7.96 \mathrm{E}-125$ & $2.38 \mathrm{E}-122$ & TWISTNB \\
\hline ENSG00000176853 & -0.991116504 & $4.73 \mathrm{E}-124$ & $1.38 \mathrm{E}-121$ & FAM91A1 \\
\hline ENSG00000113742 & -1.271922708 & $3.08 \mathrm{E}-120$ & $8.43 \mathrm{E}-118$ & CPEB4 \\
\hline ENSG00000156017 & -1.215741594 & $1.39 \mathrm{E}-117$ & $3.52 \mathrm{E}-115$ & CARNMT1 \\
\hline ENSG00000154429 & -1.346899288 & $2.62 \mathrm{E}-117$ & $6.5 \mathrm{E}-115$ & CCSAP \\
\hline ENSG00000101974 & -1.060939839 & $1.68 \mathrm{E}-116$ & $4.02 \mathrm{E}-114$ & ATP11C \\
\hline ENSG00000073712 & -0.991731657 & $6.59 \mathrm{E}-116$ & $1.54 \mathrm{E}-113$ & FERMT2 \\
\hline ENSG00000162104 & -1.444767007 & $1.64 \mathrm{E}-113$ & $3.77 \mathrm{E}-111$ & ADCY9 \\
\hline ENSG00000064042 & -1.111524916 & $1.06 \mathrm{E}-110$ & $2.36 \mathrm{E}-108$ & LIMCH1 \\
\hline ENSG00000087086 & -0.890490359 & $4.05 \mathrm{E}-108$ & $8.57 \mathrm{E}-106$ & FTL \\
\hline ENSG00000106460 & -1.091704144 & $6.11 \mathrm{E}-108$ & $1.27 \mathrm{E}-105$ & TMEM106B \\
\hline ENSG00000095752 & -1.227515654 & $8.77 \mathrm{E}-108$ & $1.8 \mathrm{E}-105$ & IL11 \\
\hline ENSG00000135521 & -1.168276042 & $5.89 \mathrm{E}-106$ & $1.12 \mathrm{E}-103$ & LTV1 \\
\hline ENSG00000187908 & -1.211596715 & $7.57 \mathrm{E}-106$ & $1.42 \mathrm{E}-103$ & DMBT1 \\
\hline ENSG00000176788 & -1.162731668 & $1.53 \mathrm{E}-103$ & $2.82 \mathrm{E}-101$ & BASP1 \\
\hline ENSG00000104375 & -1.261014996 & $2.57 \mathrm{E}-102$ & $4.69 \mathrm{E}-100$ & STK3 \\
\hline ENSG00000146143 & -1.074303191 & $9.78 \mathrm{E}-102$ & $1.76 \mathrm{E}-99$ & PRIM2 \\
\hline ENSG00000145284 & -1.323720519 & $4.77 \mathrm{E}-100$ & $8.34 \mathrm{E}-98$ & SCD5 \\
\hline ENSG00000113645 & -0.916653973 & $2.11 \mathrm{E}-99$ & $3.65 \mathrm{E}-97$ & WWC1 \\
\hline ENSG00000163161 & -1.018873601 & $3.41 \mathrm{E}-97$ & $5.59 \mathrm{E}-95$ & ERCC3 \\
\hline ENSG00000184007 & -0.822555541 & $1.26 \mathrm{E}-96$ & $2.02 \mathrm{E}-94$ & РTP4A2 \\
\hline ENSG00000143977 & -0.987888082 & $2.7 \mathrm{E}-93$ & $4.07 \mathrm{E}-91$ & SNRPG \\
\hline ENSG00000141994 & -1.367808305 & $2.61 \mathrm{E}-92$ & $3.9 \mathrm{E}-90$ & DUS3L \\
\hline ENSG00000172954 & -1.050710392 & $5.52 \mathrm{E}-92$ & $8.14 \mathrm{E}-90$ & LCLAT1 \\
\hline ENSG00000180694 & -1.033774023 & $2.99 \mathrm{E}-91$ & $4.36 \mathrm{E}-89$ & TMEM64 \\
\hline ENSG00000101003 & -1.071829328 & $3.19 \mathrm{E}-89$ & $4.36 \mathrm{E}-87$ & GINS1 \\
\hline ENSG00000123689 & -0.87877304 & $8.1 \mathrm{E}-88$ & $1.07 \mathrm{E}-85$ & G0S2 \\
\hline ENSG00000108561 & -0.906168536 & $7.24 \mathrm{E}-87$ & $9.51 \mathrm{E}-85$ & C1QBP \\
\hline ENSG00000175348 & -1.039373012 & $2.22 \mathrm{E}-85$ & $2.75 \mathrm{E}-83$ & TMEM9B \\
\hline ENSG00000011201 & -1.309694851 & $3.72 \mathrm{E}-85$ & $4.57 \mathrm{E}-83$ & ANOS1 \\
\hline ENSG00000097033 & -0.780495141 & $1.21 \mathrm{E}-84$ & $1.46 \mathrm{E}-82$ & SH3GLB1 \\
\hline
\end{tabular}


Table 3 (continued)

\begin{tabular}{|c|c|c|c|c|}
\hline Locus & $\log \mathrm{FC}$ & $P$-Value & FDR & Gene name \\
\hline ENSG00000103495 & -0.812954992 & $2.8 \mathrm{E}-83$ & $3.28 \mathrm{E}-81$ & MAZ \\
\hline ENSG00000109084 & -1.036628155 & $7.56 \mathrm{E}-83$ & $8.79 \mathrm{E}-81$ & TMEM97 \\
\hline ENSG00000170248 & -0.756074633 & $1.33 \mathrm{E}-82$ & $1.53 \mathrm{E}-80$ & PDCD6IP \\
\hline ENSG00000154734 & -0.993464022 & $2.81 \mathrm{E}-80$ & $3.07 \mathrm{E}-78$ & ADAMTS1 \\
\hline ENSG00000198959 & -0.78388823 & $3.88 \mathrm{E}-80$ & $4.17 \mathrm{E}-78$ & TGM2 \\
\hline ENSG00000109270 & -1.102716006 & $5.57 \mathrm{E}-79$ & $5.9 \mathrm{E}-77$ & LAMTOR3 \\
\hline ENSG00000171033 & -1.511641065 & $3.88 \mathrm{E}-78$ & $4.07 \mathrm{E}-76$ & PKIA \\
\hline ENSG00000262919 & -1.286604883 & $1.12 \mathrm{E}-77$ & $1.16 \mathrm{E}-75$ & FAM58A \\
\hline ENSG00000076003 & -0.895743892 & $1.38 \mathrm{E}-76$ & $1.39 \mathrm{E}-74$ & MCM6 \\
\hline ENSG00000104164 & -0.880674477 & $1.48 \mathrm{E}-76$ & $1.47 \mathrm{E}-74$ & BLOC1S6 \\
\hline ENSG00000112149 & -1.349898585 & $1.17 \mathrm{E}-75$ & $1.15 \mathrm{E}-73$ & $\mathrm{CD} 83$ \\
\hline ENSG00000173110 & -1.621696267 & $2.04 \mathrm{E}-75$ & $1.97 \mathrm{E}-73$ & HSPA6 \\
\hline ENSG00000196865 & -1.052977006 & $1.1 \mathrm{E}-74$ & $1.06 \mathrm{E}-72$ & NHLRC2 \\
\hline ENSG00000164209 & -0.79794111 & $1.25 \mathrm{E}-74$ & $1.19 \mathrm{E}-72$ & SLC25A46 \\
\hline ENSG00000092853 & -0.745590308 & $2.04 \mathrm{E}-73$ & $1.89 \mathrm{E}-71$ & CLSPN \\
\hline ENSG00000163513 & -0.719014341 & $2.71 \mathrm{E}-73$ & $2.48 \mathrm{E}-71$ & TGFBR2 \\
\hline ENSG00000127314 & -0.959251127 & $2.17 \mathrm{E}-72$ & $1.93 \mathrm{E}-70$ & RAP1B \\
\hline ENSG00000005020 & -0.909609789 & $2.38 \mathrm{E}-71$ & $2.04 \mathrm{E}-69$ & SKAP2 \\
\hline ENSG00000003989 & -1.062977014 & $3.54 \mathrm{E}-71$ & $3.02 \mathrm{E}-69$ & SLC7A2 \\
\hline ENSG00000076248 & -0.818890935 & $7.73 \mathrm{E}-70$ & $6.43 \mathrm{E}-68$ & UNG \\
\hline ENSG00000110104 & -0.886356935 & $8.21 \mathrm{E}-70$ & $6.74 \mathrm{E}-68$ & CCDC86 \\
\hline ENSG00000106034 & -2.258112177 & $2.58 \mathrm{E}-69$ & $2.1 \mathrm{E}-67$ & CPED1 \\
\hline ENSG00000139793 & -0.822257564 & $3.6 \mathrm{E}-69$ & $2.92 \mathrm{E}-67$ & MBNL2 \\
\hline ENSG00000122870 & -1.265770623 & $1.9 \mathrm{E}-68$ & $1.5 \mathrm{E}-66$ & $\mathrm{BICC} 1$ \\
\hline ENSG00000188811 & -1.270791643 & $4.79 \mathrm{E}-68$ & $3.75 \mathrm{E}-66$ & NHLRC3 \\
\hline ENSG00000140262 & -0.845128144 & $4.91 \mathrm{E}-68$ & $3.81 \mathrm{E}-66$ & TCF12 \\
\hline ENSG00000171791 & -1.383509833 & $6.73 \mathrm{E}-68$ & $5.2 \mathrm{E}-66$ & BCL2 \\
\hline ENSG00000085449 & -0.807391186 & $7.7 \mathrm{E}-68$ & $5.92 \mathrm{E}-66$ & WDFY1 \\
\hline ENSG00000163527 & -0.680451793 & $8.38 \mathrm{E}-68$ & $6.36 \mathrm{E}-66$ & STT3B \\
\hline ENSG00000101856 & -0.725495533 & $9.47 \mathrm{E}-68$ & $7.15 \mathrm{E}-66$ & PGRMC1 \\
\hline ENSG00000198689 & -0.855931203 & $1.38 \mathrm{E}-67$ & $1.04 \mathrm{E}-65$ & SLC9A6 \\
\hline ENSG00000139921 & -0.707226796 & $1.81 \mathrm{E}-67$ & $1.35 \mathrm{E}-65$ & TMX1 \\
\hline ENSG00000146047 & -0.927456112 & $2.17 \mathrm{E}-67$ & $1.61 \mathrm{E}-65$ & HIST1H2BA \\
\hline ENSG00000171867 & -0.693918536 & $2.33 \mathrm{E}-67$ & $1.72 \mathrm{E}-65$ & PRNP \\
\hline ENSG00000100625 & -0.866022602 & $5.2 \mathrm{E}-67$ & $3.79 \mathrm{E}-65$ & SIX4 \\
\hline ENSG00000160208 & -0.734399871 & $3.02 \mathrm{E}-66$ & $2.15 \mathrm{E}-64$ & RRP1B \\
\hline ENSG00000153132 & -0.846027423 & $3.16 \mathrm{E}-66$ & $2.24 \mathrm{E}-64$ & CLGN \\
\hline ENSG00000151151 & -1.049984787 & $3.44 \mathrm{E}-66$ & $2.43 \mathrm{E}-64$ & IPMK \\
\hline ENSG00000198948 & -1.324799997 & $4.2 \mathrm{E}-66$ & $2.95 \mathrm{E}-64$ & MFAP3L \\
\hline ENSG00000213160 & -1.153831739 & $4.97 \mathrm{E}-66$ & $3.45 \mathrm{E}-64$ & KLHL23 \\
\hline ENSG00000168615 & -0.756543804 & $7.94 \mathrm{E}-66$ & $5.48 \mathrm{E}-64$ & ADAM9 \\
\hline ENSG00000198964 & -0.81917694 & $1.48 \mathrm{E}-65$ & $1.02 \mathrm{E}-63$ & SGMS1 \\
\hline ENSG00000111371 & -0.655358043 & $6.25 \mathrm{E}-65$ & $4.28 \mathrm{E}-63$ & SLC38A1 \\
\hline ENSG00000138756 & -0.727021119 & $3.81 \mathrm{E}-64$ & $2.55 \mathrm{E}-62$ & BMP2K \\
\hline ENSG00000274997 & -0.669796522 & $2.01 \mathrm{E}-63$ & $1.33 \mathrm{E}-61$ & HIST1H2AH \\
\hline ENSG00000166128 & -0.743923809 & $3.52 \mathrm{E}-63$ & $2.29 \mathrm{E}-61$ & RAB8B \\
\hline ENSG00000153130 & -0.71036132 & $7.22 \mathrm{E}-63$ & $4.65 \mathrm{E}-61$ & SCOC \\
\hline
\end{tabular}


Table 3 (continued)

\begin{tabular}{|c|c|c|c|c|}
\hline Locus & $\log \mathrm{FC}$ & $P$-Value & FDR & Gene name \\
\hline ENSG00000058056 & -0.791211898 & $9.57 \mathrm{E}-63$ & $6.07 \mathrm{E}-61$ & USP13 \\
\hline ENSG00000183598 & -0.937984455 & $1.22 \mathrm{E}-62$ & $7.72 \mathrm{E}-61$ & HIST2H3D \\
\hline ENSG00000157657 & -1.331037104 & $6.49 \mathrm{E}-62$ & $4.06 \mathrm{E}-60$ & ZNF618 \\
\hline ENSG00000109586 & -0.806715459 & $3.03 \mathrm{E}-61$ & $1.89 \mathrm{E}-59$ & GALNT7 \\
\hline ENSG00000144354 & -1.284264102 & $8.96 \mathrm{E}-61$ & $5.55 \mathrm{E}-59$ & CDCA7 \\
\hline ENSG00000164211 & -0.821415196 & $9.8 \mathrm{E}-61$ & $6.04 \mathrm{E}-59$ & STARD4 \\
\hline ENSG00000099901 & -0.797049931 & $1.04 \mathrm{E}-60$ & $6.37 \mathrm{E}-59$ & RANBP1 \\
\hline ENSG00000103342 & -0.665272247 & $1.86 \mathrm{E}-60$ & $1.13 \mathrm{E}-58$ & GSPT1 \\
\hline ENSG00000273703 & -0.759808174 & $1.89 \mathrm{E}-60$ & $1.14 \mathrm{E}-58$ & HIST1H2BM \\
\hline ENSG00000162613 & -0.785667152 & $3.15 \mathrm{E}-60$ & $1.88 \mathrm{E}-58$ & FUBP1 \\
\hline ENSG00000156802 & -0.643909783 & $3.42 \mathrm{E}-60$ & $2.03 \mathrm{E}-58$ & ATAD2 \\
\hline ENSG00000107854 & -0.712659437 & $8.75 \mathrm{E}-60$ & $5.13 \mathrm{E}-58$ & TNKS2 \\
\hline ENSG00000030304 & -1.71173144 & $1.31 \mathrm{E}-59$ & $7.55 \mathrm{E}-58$ & MUSK \\
\hline ENSG00000264364 & -0.726395847 & $2.1 \mathrm{E}-59$ & $1.19 \mathrm{E}-57$ & DYNLL2 \\
\hline ENSG00000131016 & -0.791953337 & $2.29 \mathrm{E}-59$ & $1.29 \mathrm{E}-57$ & AKAP12 \\
\hline ENSG00000115364 & -0.778545058 & $2.65 \mathrm{E}-59$ & $1.48 \mathrm{E}-57$ & MRPL19 \\
\hline ENSG00000111666 & -0.804368606 & $8.4 \mathrm{E}-59$ & $4.64 \mathrm{E}-57$ & CHPT1 \\
\hline ENSG00000106771 & -0.644642299 & $1.24 \mathrm{E}-58$ & $6.77 \mathrm{E}-57$ & TMEM245 \\
\hline ENSG00000154553 & -2.010549714 & $1.69 \mathrm{E}-58$ & $9.16 \mathrm{E}-57$ & PDLIM3 \\
\hline ENSG00000180957 & -0.842836946 & $2.11 \mathrm{E}-58$ & $1.14 \mathrm{E}-56$ & PITPNB \\
\hline ENSG00000164081 & -0.810106876 & $4.81 \mathrm{E}-58$ & $2.58 \mathrm{E}-56$ & TEX264 \\
\hline ENSG00000112118 & -0.639768587 & $5.17 \mathrm{E}-58$ & $2.75 \mathrm{E}-56$ & MCM3 \\
\hline ENSG00000189057 & -0.826299148 & $7.77 \mathrm{E}-58$ & $4.09 \mathrm{E}-56$ & FAM111B \\
\hline ENSG00000116095 & -0.885698839 & $4.64 \mathrm{E}-57$ & $2.43 \mathrm{E}-55$ & PLEKHA3 \\
\hline ENSG00000113083 & -1.085829177 & $4.8 \mathrm{E}-57$ & $2.5 \mathrm{E}-55$ & LOX \\
\hline ENSG00000165156 & -0.739479342 & $5.04 \mathrm{E}-57$ & $2.62 \mathrm{E}-55$ & ZHX1 \\
\hline ENSG00000113448 & -0.76465591 & $5.39 \mathrm{E}-57$ & $2.79 \mathrm{E}-55$ & PDE4D \\
\hline ENSG00000166260 & -0.917921133 & $1.07 \mathrm{E}-56$ & $5.44 \mathrm{E}-55$ & COX11 \\
\hline ENSG00000203668 & -0.623298669 & $1.52 \mathrm{E}-56$ & $7.69 \mathrm{E}-55$ & CHML \\
\hline ENSG00000105698 & -0.737929868 & $1.71 \mathrm{E}-56$ & $8.62 \mathrm{E}-55$ & USF2 \\
\hline ENSG00000176890 & -0.736138571 & $2.01 \mathrm{E}-56$ & $1.01 \mathrm{E}-54$ & TYMS \\
\hline ENSG00000164466 & -0.68314086 & $2.1 \mathrm{E}-56$ & $1.05 \mathrm{E}-54$ & SFXN1 \\
\hline ENSG00000197312 & -0.84172031 & $7.43 \mathrm{E}-56$ & $3.67 \mathrm{E}-54$ & DDI2 \\
\hline ENSG00000132581 & -0.797529275 & $1.08 \mathrm{E}-55$ & $5.31 \mathrm{E}-54$ & SDF2 \\
\hline ENSG00000105281 & -0.76012066 & $1.34 \mathrm{E}-54$ & $6.44 \mathrm{E}-53$ & SLC1A5 \\
\hline ENSG00000101773 & -0.684417581 & $1.43 \mathrm{E}-54$ & $6.8 \mathrm{E}-53$ & RBBP8 \\
\hline ENSG00000125166 & -0.64746293 & $2.47 \mathrm{E}-54$ & $1.17 \mathrm{E}-52$ & GOT2 \\
\hline ENSG00000065615 & -0.766061917 & $3.65 \mathrm{E}-54$ & $1.72 \mathrm{E}-52$ & CYB5R4 \\
\hline ENSG00000106366 & -0.690600823 & $4.53 \mathrm{E}-54$ & $2.12 \mathrm{E}-52$ & SERPINE1 \\
\hline ENSG00000138675 & -1.055137884 & $2.97 \mathrm{E}-53$ & $1.37 \mathrm{E}-51$ & FGF5 \\
\hline ENSG00000166801 & -0.679971393 & $4.81 \mathrm{E}-53$ & $2.18 \mathrm{E}-51$ & FAM111A \\
\hline ENSG00000125430 & -1.119040342 & $1.8 \mathrm{E}-52$ & $8.13 \mathrm{E}-51$ & HS3ST3B1 \\
\hline ENSG00000136986 & -0.64205774 & $3.04 \mathrm{E}-52$ & $1.37 \mathrm{E}-50$ & DERL1 \\
\hline ENSG00000143507 & -0.824435143 & $3.91 \mathrm{E}-52$ & $1.75 \mathrm{E}-50$ & DUSP10 \\
\hline ENSG00000132646 & -0.659226998 & $4.16 \mathrm{E}-52$ & $1.85 \mathrm{E}-50$ & PCNA \\
\hline ENSG00000092470 & -0.776503363 & $4.36 \mathrm{E}-52$ & $1.93 \mathrm{E}-50$ & WDR76 \\
\hline ENSG00000125870 & -0.692582004 & $6.21 \mathrm{E}-52$ & $2.72 \mathrm{E}-50$ & SNRPB2 \\
\hline
\end{tabular}


Table 3 (continued)

\begin{tabular}{|c|c|c|c|c|}
\hline Locus & $\log \mathrm{FC}$ & $P$-Value & FDR & Gene name \\
\hline ENSG00000130830 & -1.001331439 & $7.01 \mathrm{E}-52$ & $3.05 \mathrm{E}-50$ & MPP1 \\
\hline ENSG00000169193 & -1.233765255 & $1.18 \mathrm{E}-51$ & $5.09 \mathrm{E}-50$ & $\mathrm{CCDC} 126$ \\
\hline ENSG00000115159 & -0.745203813 & $1.55 \mathrm{E}-51$ & $6.63 \mathrm{E}-50$ & GPD2 \\
\hline ENSG00000089597 & -0.608914143 & $4.59 \mathrm{E}-51$ & $1.95 \mathrm{E}-49$ & GANAB \\
\hline ENSG00000138448 & -0.679300714 & $2.25 \mathrm{E}-50$ & $9.46 \mathrm{E}-49$ & ITGAV \\
\hline ENSG00000261609 & -0.838562302 & $3.25 \mathrm{E}-50$ & $1.36 \mathrm{E}-48$ & GAN \\
\hline ENSG00000118596 & -0.91558522 & $3.25 \mathrm{E}-50$ & $1.36 \mathrm{E}-48$ & SLC16A7 \\
\hline ENSG00000104388 & -0.77698668 & $8.4 \mathrm{E}-50$ & $3.46 \mathrm{E}-48$ & RAB2A \\
\hline ENSG00000111725 & -0.851677774 & $1.59 \mathrm{E}-49$ & $6.47 \mathrm{E}-48$ & PRKAB1 \\
\hline ENSG00000070214 & -0.695894872 & $1.61 \mathrm{E}-49$ & $6.52 \mathrm{E}-48$ & SLC44A1 \\
\hline ENSG00000149289 & -0.717233099 & $1.62 \mathrm{E}-49$ & $6.53 \mathrm{E}-48$ & $\mathrm{ZC} 3 \mathrm{H} 12 \mathrm{C}$ \\
\hline ENSG00000196323 & -0.700131838 & $1.75 \mathrm{E}-49$ & $7.04 \mathrm{E}-48$ & ZBTB44 \\
\hline ENSG00000182504 & -0.657051624 & $8.72 \mathrm{E}-49$ & $3.46 \mathrm{E}-47$ & CEP97 \\
\hline ENSG00000082269 & -0.721562816 & $2.1 \mathrm{E}-48$ & $8.21 \mathrm{E}-47$ & FAM135A \\
\hline ENSG00000055208 & -0.688892188 & $2.44 \mathrm{E}-48$ & $9.51 \mathrm{E}-47$ & TAB2 \\
\hline ENSG00000083720 & -0.85708639 & $3.3 \mathrm{E}-48$ & $1.28 \mathrm{E}-46$ & OXCT1 \\
\hline ENSG00000110031 & -0.750834355 & $3.95 \mathrm{E}-48$ & $1.53 \mathrm{E}-46$ & LPXN \\
\hline ENSG00000121966 & -0.877794416 & $4.44 \mathrm{E}-48$ & $1.72 \mathrm{E}-46$ & CXCR4 \\
\hline ENSG00000081923 & -0.788155108 & $8.6 \mathrm{E}-48$ & $3.3 \mathrm{E}-46$ & АТP8B1 \\
\hline ENSG00000133026 & -0.759331662 & $3.21 \mathrm{E}-47$ & $1.21 \mathrm{E}-45$ & MYH10 \\
\hline ENSG00000167645 & -0.945756835 & $3.93 \mathrm{E}-47$ & $1.47 \mathrm{E}-45$ & YIF1B \\
\hline ENSG00000048392 & -0.69447929 & $5.19 \mathrm{E}-47$ & $1.93 \mathrm{E}-45$ & RRM2B \\
\hline ENSG00000157978 & -0.881827291 & $9.73 \mathrm{E}-47$ & $3.61 \mathrm{E}-45$ & LDLRAP1 \\
\hline ENSG00000169429 & -0.814790283 & $1.09 \mathrm{E}-46$ & $4.01 \mathrm{E}-45$ & CXCL8 \\
\hline ENSG00000253304 & -1.283748113 & $2.32 \mathrm{E}-46$ & $8.48 \mathrm{E}-45$ & TMEM200B \\
\hline ENSG00000139278 & -0.735325809 & $3.22 \mathrm{E}-46$ & $1.18 \mathrm{E}-44$ & GLIPR1 \\
\hline ENSG00000164070 & -0.61500254 & $3.29 \mathrm{E}-46$ & $1.2 \mathrm{E}-44$ & HSPA4L \\
\hline ENSG00000116406 & -0.582479865 & $3.37 \mathrm{E}-46$ & $1.22 \mathrm{E}-44$ & EDEM3 \\
\hline ENSG00000205302 & -0.632567484 & $6.01 \mathrm{E}-46$ & $2.18 \mathrm{E}-44$ & SNX2 \\
\hline ENSG00000119917 & -0.598753864 & $1.72 \mathrm{E}-45$ & $6.12 \mathrm{E}-44$ & IFIT3 \\
\hline ENSG00000094804 & -0.61234381 & $2.51 \mathrm{E}-45$ & $8.93 \mathrm{E}-44$ & CDC6 \\
\hline ENSG00000180998 & -1.39647951 & $2.96 \mathrm{E}-45$ & $1.05 \mathrm{E}-43$ & GPR137C \\
\hline ENSG00000166741 & -1.457281718 & $1.07 \mathrm{E}-44$ & $3.74 \mathrm{E}-43$ & NNMT \\
\hline ENSG00000113070 & -0.759564729 & $1.28 \mathrm{E}-44$ & $4.43 \mathrm{E}-43$ & HBEGF \\
\hline ENSG00000135250 & -0.766365183 & $1.37 \mathrm{E}-44$ & $4.74 \mathrm{E}-43$ & SRPK2 \\
\hline ENSG00000106683 & -0.770624188 & $2.57 \mathrm{E}-44$ & $8.9 \mathrm{E}-43$ & LIMK1 \\
\hline ENSG00000214517 & -0.683773756 & $2.59 \mathrm{E}-44$ & $8.93 \mathrm{E}-43$ & PPME1 \\
\hline ENSG00000100479 & -0.857727695 & $2.8 \mathrm{E}-44$ & $9.64 \mathrm{E}-43$ & POLE2 \\
\hline ENSG00000130175 & -0.636724289 & $5.94 \mathrm{E}-44$ & $2.02 \mathrm{E}-42$ & PRKCSH \\
\hline ENSG00000170185 & -0.607338959 & $1.22 \mathrm{E}-43$ & $4.08 \mathrm{E}-42$ & USP38 \\
\hline ENSG00000123908 & -0.609276107 & $1.75 \mathrm{E}-43$ & $5.86 \mathrm{E}-42$ & 37469 \\
\hline ENSG00000064666 & -0.604102394 & $1.93 \mathrm{E}-43$ & $6.43 \mathrm{E}-42$ & CNN2 \\
\hline ENSG00000130024 & -0.624632149 & $4.42 \mathrm{E}-43$ & $1.45 \mathrm{E}-41$ & PHF10 \\
\hline ENSG00000135272 & -0.779876332 & $1.35 \mathrm{E}-42$ & $4.34 \mathrm{E}-41$ & MDFIC \\
\hline ENSG00000138646 & -0.611105347 & $1.87 \mathrm{E}-42$ & $5.96 \mathrm{E}-41$ & HERC5 \\
\hline ENSG00000178904 & -0.821536016 & $4.05 \mathrm{E}-42$ & $1.28 \mathrm{E}-40$ & DPY19L3 \\
\hline ENSG00000116984 & -0.592452679 & $6.84 \mathrm{E}-42$ & $2.15 \mathrm{E}-40$ & MTR \\
\hline
\end{tabular}


Table 3 (continued)

\begin{tabular}{|c|c|c|c|c|}
\hline Locus & $\log \mathrm{FC}$ & $P$-Value & FDR & Gene name \\
\hline ENSG00000151233 & -0.696734208 & $1.24 \mathrm{E}-41$ & $3.89 \mathrm{E}-40$ & GXYLT1 \\
\hline ENSG00000049130 & -1.363755139 & $2.4 \mathrm{E}-41$ & $7.47 \mathrm{E}-40$ & KITLG \\
\hline ENSG00000131943 & -0.819671981 & $4.72 \mathrm{E}-41$ & $1.45 \mathrm{E}-39$ & C19orf12 \\
\hline ENSG00000180730 & -0.972346863 & $6.99 \mathrm{E}-41$ & $2.14 \mathrm{E}-39$ & SHISA2 \\
\hline ENSG00000151239 & -0.646933517 & $1.79 \mathrm{E}-40$ & $5.44 \mathrm{E}-39$ & TWF1 \\
\hline ENSG00000132819 & -0.768136766 & $2.14 \mathrm{E}-40$ & $6.44 \mathrm{E}-39$ & RBM38 \\
\hline ENSG00000163577 & -0.691799144 & $2.24 \mathrm{E}-40$ & $6.7 \mathrm{E}-39$ & EIF5A2 \\
\hline ENSG00000145743 & -0.871378383 & $2.77 \mathrm{E}-40$ & $8.28 \mathrm{E}-39$ & FBXL17 \\
\hline ENSG00000137168 & -0.580135569 & $9.42 \mathrm{E}-40$ & $2.77 \mathrm{E}-38$ & PPIL1 \\
\hline ENSG00000038210 & -0.645756993 & $1.75 \mathrm{E}-39$ & $5.06 \mathrm{E}-38$ & PI4K2B \\
\hline ENSG00000146263 & -0.647666403 & $1.86 \mathrm{E}-39$ & $5.35 \mathrm{E}-38$ & MMS22L \\
\hline ENSG00000116717 & -0.675446031 & $1.93 \mathrm{E}-39$ & $5.56 \mathrm{E}-38$ & GADD45A \\
\hline ENSG00000167842 & -0.621928208 & $1.97 \mathrm{E}-39$ & $5.64 \mathrm{E}-38$ & MIS12 \\
\hline ENSG00000114520 & -0.763326165 & $2.34 \mathrm{E}-39$ & $6.7 \mathrm{E}-38$ & SNX4 \\
\hline ENSG00000154678 & -0.944300821 & $2.5 \mathrm{E}-39$ & $7.13 \mathrm{E}-38$ & PDE1C \\
\hline ENSG00000164402 & -0.637231639 & $4.65 \mathrm{E}-39$ & $1.31 \mathrm{E}-37$ & 39692 \\
\hline ENSG00000162073 & -0.831857412 & $5.7 \mathrm{E}-39$ & $1.6 \mathrm{E}-37$ & PAQR4 \\
\hline ENSG00000144369 & -0.757917112 & $8.73 E-39$ & $2.43 \mathrm{E}-37$ & FAM171B \\
\hline ENSG00000123572 & -1.320658507 & $8.85 \mathrm{E}-39$ & $2.46 \mathrm{E}-37$ & NRK \\
\hline ENSG00000129691 & -0.662466725 & $1.08 \mathrm{E}-38$ & $2.99 \mathrm{E}-37$ & $\mathrm{ASH} 2 \mathrm{~L}$ \\
\hline ENSG00000002822 & -0.607342874 & $1.85 \mathrm{E}-38$ & $5.06 \mathrm{E}-37$ & MAD1L1 \\
\hline ENSG00000101412 & -0.797853061 & $2.45 \mathrm{E}-38$ & $6.64 \mathrm{E}-37$ & $\mathrm{E} 2 \mathrm{~F} 1$ \\
\hline ENSG00000156162 & -0.585388505 & $2.98 \mathrm{E}-38$ & $8.02 \mathrm{E}-37$ & DPY19L4 \\
\hline ENSG00000112144 & -0.601617859 & $3.85 \mathrm{E}-38$ & $1.03 \mathrm{E}-36$ & ICK \\
\hline ENSG00000125734 & -0.622236713 & $3.88 \mathrm{E}-38$ & $1.04 \mathrm{E}-36$ & GPR108 \\
\hline ENSG00000101544 & -0.625762283 & $3.98 \mathrm{E}-38$ & $1.06 \mathrm{E}-36$ & ADNP2 \\
\hline ENSG00000198860 & -0.623117034 & $4.95 \mathrm{E}-38$ & $1.31 \mathrm{E}-36$ & TSEN15 \\
\hline ENSG00000091986 & -0.628100732 & $5.14 \mathrm{E}-38$ & $1.36 \mathrm{E}-36$ & CCDC80 \\
\hline ENSG00000114982 & -0.612077259 & $9.28 \mathrm{E}-38$ & $2.42 \mathrm{E}-36$ & KANSL3 \\
\hline ENSG00000145604 & -0.706553217 & $1.28 \mathrm{E}-37$ & $3.32 \mathrm{E}-36$ & SKP2 \\
\hline ENSG00000187051 & -0.687870702 & $1.56 \mathrm{E}-37$ & $4.04 \mathrm{E}-36$ & RPS19BP1 \\
\hline ENSG00000163626 & -0.701696175 & $2.3 \mathrm{E}-37$ & $5.91 \mathrm{E}-36$ & COX18 \\
\hline ENSG00000125885 & -0.583920903 & $2.3 \mathrm{E}-37$ & $5.91 \mathrm{E}-36$ & MCM8 \\
\hline ENSG00000157557 & -0.662994439 & $4.57 \mathrm{E}-37$ & $1.15 \mathrm{E}-35$ & ETS2 \\
\hline ENSG00000127564 & -0.830827192 & $4.99 \mathrm{E}-37$ & $1.26 \mathrm{E}-35$ & PKMYT1 \\
\hline ENSG00000172260 & -0.882667662 & $5.77 \mathrm{E}-37$ & $1.45 \mathrm{E}-35$ & NEGR1 \\
\hline ENSG00000198478 & -0.913187859 & $7.59 \mathrm{E}-37$ & $1.9 \mathrm{E}-35$ & SH3BGRL2 \\
\hline ENSG00000152455 & -0.651372389 & $9.5 \mathrm{E}-37$ & $2.37 \mathrm{E}-35$ & SUV39H2 \\
\hline ENSG00000181744 & -0.857381192 & $3.74 \mathrm{E}-36$ & $9.25 \mathrm{E}-35$ & C3orf58 \\
\hline ENSG00000105825 & -0.683206742 & $4.19 \mathrm{E}-36$ & $1.03 \mathrm{E}-34$ & TFPI2 \\
\hline ENSG00000185129 & -0.650813837 & $4.66 \mathrm{E}-36$ & $1.14 \mathrm{E}-34$ & PURA \\
\hline ENSG00000131153 & -0.830497048 & $5.14 \mathrm{E}-36$ & $1.26 \mathrm{E}-34$ & GINS2 \\
\hline ENSG00000104361 & -1.128506294 & $9.17 \mathrm{E}-36$ & $2.23 \mathrm{E}-34$ & NIPAL2 \\
\hline ENSG00000142552 & -2.164888397 & $1.32 \mathrm{E}-35$ & $3.17 \mathrm{E}-34$ & $\mathrm{RCN} 3$ \\
\hline ENSG00000118971 & -0.828403671 & $1.65 \mathrm{E}-35$ & $3.94 \mathrm{E}-34$ & CCND2 \\
\hline ENSG00000162437 & -0.911533859 & $1.86 \mathrm{E}-35$ & $4.42 \mathrm{E}-34$ & RAVER2 \\
\hline ENSG00000072609 & -0.725569758 & $2.69 \mathrm{E}-35$ & $6.35 \mathrm{E}-34$ & CHFR \\
\hline
\end{tabular}


Table 3 (continued)

\begin{tabular}{|c|c|c|c|c|}
\hline Locus & $\log \mathrm{FC}$ & $P$-Value & FDR & Gene name \\
\hline ENSG00000135750 & -1.097089858 & $2.74 \mathrm{E}-35$ & $6.47 \mathrm{E}-34$ & KCNK1 \\
\hline ENSG00000198108 & -1.330709511 & $3.6 \mathrm{E}-35$ & $8.47 \mathrm{E}-34$ & CHSY3 \\
\hline ENSG00000139438 & -1.36818776 & $5.27 \mathrm{E}-35$ & $1.23 \mathrm{E}-33$ & FAM222A \\
\hline ENSG00000176170 & -1.061371315 & $1.42 \mathrm{E}-34$ & $3.25 \mathrm{E}-33$ & SPHK1 \\
\hline ENSG00000172159 & -0.719708766 & $1.54 \mathrm{E}-34$ & $3.53 \mathrm{E}-33$ & FRMD3 \\
\hline ENSG00000115380 & -0.592899689 & $2.06 \mathrm{E}-34$ & $4.68 \mathrm{E}-33$ & EFEMP1 \\
\hline ENSG00000109511 & -1.823866992 & $2.43 \mathrm{E}-34$ & $5.52 \mathrm{E}-33$ & ANXA10 \\
\hline ENSG00000198780 & -0.894526335 & $2.96 \mathrm{E}-34$ & $6.7 \mathrm{E}-33$ & FAM169A \\
\hline ENSG00000132970 & -0.851435716 & $1.4 \mathrm{E}-33$ & $3.08 \mathrm{E}-32$ & WASF3 \\
\hline ENSG00000136111 & -0.603181977 & $1.96 \mathrm{E}-33$ & $4.27 \mathrm{E}-32$ & TBC1D4 \\
\hline ENSG00000225830 & -0.709999482 & $2.48 \mathrm{E}-33$ & $5.35 \mathrm{E}-32$ & ERCC6 \\
\hline ENSG00000109255 & -1.175751743 & $3.49 \mathrm{E}-33$ & $7.47 \mathrm{E}-32$ & NMU \\
\hline ENSG00000121058 & -0.618925976 & $4.93 \mathrm{E}-33$ & $1.05 \mathrm{E}-31$ & COIL \\
\hline ENSG00000164508 & -1.194336423 & $7.79 \mathrm{E}-33$ & $1.64 \mathrm{E}-31$ & HIST1H2AA \\
\hline ENSG00000123570 & -1.292915486 & $7.81 \mathrm{E}-33$ & $1.65 \mathrm{E}-31$ & RAB9B \\
\hline ENSG00000176834 & -0.652497435 & $8.39 \mathrm{E}-33$ & $1.76 \mathrm{E}-31$ & VSIG10 \\
\hline ENSG00000186416 & -0.589099004 & $8.99 \mathrm{E}-33$ & $1.89 \mathrm{E}-31$ & NKRF \\
\hline ENSG00000106789 & -0.67263713 & $1.3 \mathrm{E}-32$ & $2.72 \mathrm{E}-31$ & $\mathrm{CORO} 2 \mathrm{~A}$ \\
\hline ENSG00000172379 & -0.788111039 & $1.74 \mathrm{E}-32$ & $3.62 \mathrm{E}-31$ & ARNT2 \\
\hline ENSG00000112592 & -0.651455796 & $1.74 \mathrm{E}-32$ & $3.62 \mathrm{E}-31$ & TBP \\
\hline ENSG00000159259 & -0.683175408 & $3.54 \mathrm{E}-32$ & $7.29 \mathrm{E}-31$ & CHAF1B \\
\hline ENSG00000226742 & -1.146129313 & $6.12 \mathrm{E}-32$ & $1.24 \mathrm{E}-30$ & HSBP1L1 \\
\hline ENSG00000129173 & -0.786345274 & $7.03 \mathrm{E}-32$ & $1.43 \mathrm{E}-30$ & $\mathrm{E} 2 \mathrm{~F} 8$ \\
\hline ENSG00000170689 & -0.891856708 & $1.2 \mathrm{E}-31$ & $2.41 \mathrm{E}-30$ & HOXB9 \\
\hline ENSG00000112294 & -1.176881508 & $1.51 \mathrm{E}-31$ & $2.99 \mathrm{E}-30$ & ALDH5A1 \\
\hline ENSG00000174021 & -0.597920622 & $2 \mathrm{E}-31$ & $3.95 \mathrm{E}-30$ & GNG5 \\
\hline ENSG00000164199 & -0.796504879 & $2.27 \mathrm{E}-31$ & $4.46 \mathrm{E}-30$ & ADGRV1 \\
\hline ENSG00000153037 & -0.672607947 & $3.19 \mathrm{E}-31$ & $6.24 \mathrm{E}-30$ & SRP19 \\
\hline ENSG00000170734 & -0.586607195 & $3.55 \mathrm{E}-31$ & $6.92 \mathrm{E}-30$ & POLH \\
\hline ENSG00000179454 & -0.750172183 & $3.78 \mathrm{E}-31$ & $7.35 \mathrm{E}-30$ & KLHL28 \\
\hline ENSG00000168564 & -0.588203808 & $4.93 \mathrm{E}-31$ & $9.54 \mathrm{E}-30$ & CDKN2AIP \\
\hline ENSG00000112031 & -0.762313636 & $6.71 \mathrm{E}-31$ & $1.29 \mathrm{E}-29$ & MTRF1L \\
\hline ENSG00000180773 & -0.580924737 & $8.38 \mathrm{E}-31$ & $1.61 \mathrm{E}-29$ & SLC36A4 \\
\hline ENSG00000164161 & -1.167949712 & $8.5 \mathrm{E}-31$ & $1.63 \mathrm{E}-29$ & HHIP \\
\hline ENSG00000170085 & -1.010340626 & $8.52 \mathrm{E}-31$ & $1.63 \mathrm{E}-29$ & SIMC1 \\
\hline ENSG00000213047 & -0.852741909 & $9.45 \mathrm{E}-31$ & $1.8 \mathrm{E}-29$ & DENND1B \\
\hline ENSG00000178695 & -0.608721797 & $1.3 \mathrm{E}-30$ & $2.45 \mathrm{E}-29$ & KCTD12 \\
\hline ENSG00000162694 & -0.581927567 & $1.4 \mathrm{E}-30$ & $2.63 \mathrm{E}-29$ & EXTL2 \\
\hline ENSG00000153993 & -1.881252549 & $2.69 \mathrm{E}-30$ & $5.02 \mathrm{E}-29$ & SEMA3D \\
\hline ENSG00000121236 & -0.974421588 & $3.3 \mathrm{E}-30$ & $6.14 \mathrm{E}-29$ & TRIM6 \\
\hline ENSG00000157869 & -0.655287956 & $4.29 \mathrm{E}-30$ & $7.95 \mathrm{E}-29$ & RAB28 \\
\hline ENSG00000135966 & -0.618380605 & $6.88 \mathrm{E}-30$ & $1.26 \mathrm{E}-28$ & TGFBRAP1 \\
\hline ENSG00000121005 & -1.212412618 & $8.16 \mathrm{E}-30$ & $1.49 \mathrm{E}-28$ & CRISPLD1 \\
\hline ENSG00000067177 & -0.643679511 & $1.08 \mathrm{E}-29$ & $1.95 \mathrm{E}-28$ & PHKA1 \\
\hline ENSG00000104643 & -0.792913093 & $1.57 \mathrm{E}-29$ & $2.8 \mathrm{E}-28$ & MTMR9 \\
\hline ENSG00000163818 & -0.711190839 & $1.7 \mathrm{E}-29$ & $3.03 \mathrm{E}-28$ & LZTFL1 \\
\hline ENSG00000145545 & -0.582387628 & $2.79 \mathrm{E}-29$ & $4.91 \mathrm{E}-28$ & SRD5A1 \\
\hline
\end{tabular}


Table 3 (continued)

\begin{tabular}{|c|c|c|c|c|}
\hline Locus & $\log \mathrm{FC}$ & $P$-Value & FDR & Gene name \\
\hline ENSG00000143376 & -0.70665512 & $3.54 \mathrm{E}-29$ & $6.2 \mathrm{E}-28$ & SNX27 \\
\hline ENSG00000276043 & -0.609555567 & $5.15 \mathrm{E}-29$ & $8.95 \mathrm{E}-28$ & UHRF1 \\
\hline ENSG00000176406 & -1.738298125 & $1.08 \mathrm{E}-28$ & $1.85 \mathrm{E}-27$ & RIMS2 \\
\hline ENSG00000101871 & -0.597281832 & $1.53 \mathrm{E}-28$ & $2.6 \mathrm{E}-27$ & MID1 \\
\hline ENSG00000197223 & -0.808372429 & $4.86 \mathrm{E}-28$ & $8.12 \mathrm{E}-27$ & C1D \\
\hline ENSG00000214357 & -0.84131965 & $5.59 \mathrm{E}-28$ & $9.31 \mathrm{E}-27$ & NEURL1B \\
\hline ENSG00000137462 & -1.051657592 & $7.74 \mathrm{E}-28$ & $1.28 \mathrm{E}-26$ & TLR2 \\
\hline ENSG00000040199 & -0.614077873 & $1.01 \mathrm{E}-27$ & $1.65 \mathrm{E}-26$ & PHLPP2 \\
\hline ENSG00000135185 & -0.890686199 & $1.23 \mathrm{E}-27$ & $2.01 \mathrm{E}-26$ & TMEM243 \\
\hline ENSG00000128591 & -0.771115601 & $1.28 \mathrm{E}-27$ & $2.1 \mathrm{E}-26$ & FLNC \\
\hline ENSG00000113356 & -0.70152701 & $1.33 \mathrm{E}-27$ & $2.17 \mathrm{E}-26$ & POLR3G \\
\hline ENSG00000112218 & -1.31975116 & $1.38 \mathrm{E}-27$ & $2.25 \mathrm{E}-26$ & GPR63 \\
\hline ENSG00000166250 & -0.584854006 & $1.41 \mathrm{E}-27$ & $2.3 \mathrm{E}-26$ & CLMP \\
\hline ENSG00000123892 & -0.709596504 & $2.05 \mathrm{E}-27$ & $3.31 \mathrm{E}-26$ & RAB38 \\
\hline ENSG00000126215 & -0.59285666 & $3.95 \mathrm{E}-27$ & $6.32 \mathrm{E}-26$ & XRCC3 \\
\hline ENSG00000111331 & -0.709429851 & $4.04 \mathrm{E}-27$ & $6.45 \mathrm{E}-26$ & OAS3 \\
\hline ENSG00000179348 & -0.959652747 & $5.77 \mathrm{E}-27$ & $9.16 \mathrm{E}-26$ & GATA2 \\
\hline ENSG00000135045 & -0.580419299 & $7.97 \mathrm{E}-27$ & $1.25 \mathrm{E}-25$ & C9orf40 \\
\hline ENSG00000128923 & -0.640712113 & $9.41 \mathrm{E}-27$ & $1.48 \mathrm{E}-25$ & MINDY2 \\
\hline ENSG00000151150 & -1.00381512 & $1.76 \mathrm{E}-26$ & $2.72 \mathrm{E}-25$ & ANK3 \\
\hline ENSG00000167513 & -0.659977018 & $3.21 \mathrm{E}-26$ & $4.9 \mathrm{E}-25$ & CDT1 \\
\hline ENSG00000084676 & -0.598801314 & $4.32 \mathrm{E}-26$ & $6.55 \mathrm{E}-25$ & NCOA1 \\
\hline ENSG00000174405 & -0.594636053 & $7.09 \mathrm{E}-26$ & $1.06 \mathrm{E}-24$ & LIG4 \\
\hline ENSG00000134056 & -0.815574482 & $1.05 \mathrm{E}-25$ & $1.57 \mathrm{E}-24$ & MRPS36 \\
\hline ENSG00000137965 & -0.582015156 & $1.21 \mathrm{E}-25$ & $1.79 \mathrm{E}-24$ & IFI44 \\
\hline ENSG00000157510 & -0.701292502 & $1.38 \mathrm{E}-25$ & $2.04 \mathrm{E}-24$ & AFAP1L1 \\
\hline ENSG00000173083 & -0.723996154 & $2.26 \mathrm{E}-25$ & $3.32 \mathrm{E}-24$ & HPSE \\
\hline ENSG00000175197 & -0.673342058 & $5.14 \mathrm{E}-25$ & $7.46 \mathrm{E}-24$ & DDIT3 \\
\hline ENSG00000108960 & -0.58542233 & $6.22 \mathrm{E}-25$ & $8.99 \mathrm{E}-24$ & MMD \\
\hline ENSG00000100916 & -0.624568654 & $1.56 \mathrm{E}-24$ & $2.22 \mathrm{E}-23$ & BRMS1L \\
\hline ENSG00000181222 & -0.726152084 & $1.6 \mathrm{E}-24$ & $2.27 \mathrm{E}-23$ & POLR2A \\
\hline ENSG00000187123 & -0.956022878 & $2.07 \mathrm{E}-24$ & $2.9 \mathrm{E}-23$ & LYPD6 \\
\hline ENSG00000124788 & -0.590242443 & $2.42 \mathrm{E}-24$ & $3.37 \mathrm{E}-23$ & ATXN1 \\
\hline ENSG00000129354 & -0.691992358 & $2.83 \mathrm{E}-24$ & $3.93 \mathrm{E}-23$ & AP1M2 \\
\hline ENSG00000078401 & -0.592475007 & $4 \mathrm{E}-24$ & $5.52 \mathrm{E}-23$ & EDN1 \\
\hline ENSG00000121316 & -1.050558857 & $6.3 \mathrm{E}-24$ & $8.57 \mathrm{E}-23$ & PLBD1 \\
\hline ENSG00000121211 & -0.582342226 & $6.37 \mathrm{E}-24$ & $8.66 \mathrm{E}-23$ & MND1 \\
\hline ENSG00000135776 & -0.585970667 & $1.14 \mathrm{E}-23$ & $1.52 \mathrm{E}-22$ & $\mathrm{ABCB} 10$ \\
\hline ENSG00000162599 & -0.648028127 & $1.15 \mathrm{E}-23$ & $1.53 \mathrm{E}-22$ & NFIA \\
\hline ENSG00000143195 & -0.652324164 & $1.34 \mathrm{E}-23$ & $1.78 \mathrm{E}-22$ & ILDR2 \\
\hline ENSG00000182010 & -0.810382882 & $1.94 \mathrm{E}-23$ & $2.57 \mathrm{E}-22$ & RTKN2 \\
\hline ENSG00000196083 & -0.719308824 & $1.98 \mathrm{E}-23$ & $2.62 \mathrm{E}-22$ & IL1RAP \\
\hline ENSG00000135698 & -0.685340754 & $2.6 \mathrm{E}-23$ & $3.41 \mathrm{E}-22$ & MPHOSPH6 \\
\hline ENSG00000122435 & -0.73861782 & $4.38 \mathrm{E}-23$ & $5.68 \mathrm{E}-22$ & TRMT13 \\
\hline ENSG00000099256 & -0.742089516 & $5.07 \mathrm{E}-23$ & $6.54 \mathrm{E}-22$ & PRTFDC1 \\
\hline ENSG00000173926 & -0.725353141 & $5.49 \mathrm{E}-23$ & $7.06 \mathrm{E}-22$ & MARCH3 \\
\hline ENSG00000171016 & -0.635078785 & $6.62 \mathrm{E}-23$ & $8.48 \mathrm{E}-22$ & PYGO1 \\
\hline
\end{tabular}


Table 3 (continued)

\begin{tabular}{|c|c|c|c|c|}
\hline Locus & $\log \mathrm{FC}$ & $P$-Value & FDR & Gene name \\
\hline ENSG00000166908 & -0.610595678 & $1.01 \mathrm{E}-22$ & $1.28 \mathrm{E}-21$ & PIP4K2C \\
\hline ENSG00000254535 & -1.198870344 & $1.2 \mathrm{E}-22$ & $1.52 \mathrm{E}-21$ & PABPC4L \\
\hline ENSG00000117481 & -0.628980367 & $1.35 \mathrm{E}-22$ & $1.69 \mathrm{E}-21$ & NSUN4 \\
\hline ENSG00000049192 & -0.719589951 & $1.56 \mathrm{E}-22$ & $1.96 \mathrm{E}-21$ & ADAMTS6 \\
\hline ENSG00000133739 & -0.688520659 & $1.78 \mathrm{E}-22$ & $2.23 \mathrm{E}-21$ & LRRCC1 \\
\hline ENSG00000083454 & -1.060807972 & $1.96 \mathrm{E}-22$ & $2.44 \mathrm{E}-21$ & P2RX5 \\
\hline ENSG00000154319 & -0.887801464 & $2.23 \mathrm{E}-22$ & $2.77 \mathrm{E}-21$ & FAM167A \\
\hline ENSG00000023892 & -0.675886174 & $2.38 \mathrm{E}-22$ & $2.95 \mathrm{E}-21$ & DEF6 \\
\hline ENSG00000138795 & -0.843440962 & $3.04 \mathrm{E}-22$ & $3.75 \mathrm{E}-21$ & LEF1 \\
\hline ENSG00000079156 & -0.842535226 & $3.07 \mathrm{E}-22$ & $3.78 \mathrm{E}-21$ & OSBPL6 \\
\hline ENSG00000170396 & -0.805881831 & $3.81 \mathrm{E}-22$ & $4.67 \mathrm{E}-21$ & ZNF804A \\
\hline ENSG00000135211 & -0.747849147 & $3.86 \mathrm{E}-22$ & $4.73 \mathrm{E}-21$ & TMEM60 \\
\hline ENSG00000165323 & -0.725561816 & $6.07 \mathrm{E}-22$ & $7.35 \mathrm{E}-21$ & FAT3 \\
\hline ENSG00000152495 & -0.751284296 & $6.71 \mathrm{E}-22$ & $8.1 \mathrm{E}-21$ & CAMK4 \\
\hline ENSG00000124813 & -0.681157995 & $7.41 \mathrm{E}-22$ & $8.93 \mathrm{E}-21$ & RUNX2 \\
\hline ENSG00000109738 & -0.716767424 & $1.08 \mathrm{E}-21$ & $1.3 \mathrm{E}-20$ & GLRB \\
\hline ENSG00000082438 & -1.400231606 & $1.49 \mathrm{E}-21$ & $1.77 \mathrm{E}-20$ & COBLL1 \\
\hline ENSG00000138463 & -0.692070234 & $1.91 \mathrm{E}-21$ & $2.25 \mathrm{E}-20$ & DIRC2 \\
\hline ENSG00000148680 & -0.6672409 & $1.96 \mathrm{E}-21$ & $2.31 \mathrm{E}-20$ & HTR7 \\
\hline ENSG00000169750 & -0.921289251 & $3.24 \mathrm{E}-21$ & $3.75 \mathrm{E}-20$ & RAC3 \\
\hline ENSG00000114698 & -1.130704953 & $3.84 \mathrm{E}-21$ & $4.42 \mathrm{E}-20$ & PLSCR4 \\
\hline ENSG00000112319 & -0.822410931 & $6.03 \mathrm{E}-21$ & $6.89 \mathrm{E}-20$ & EYA4 \\
\hline ENSG00000115392 & -0.58789192 & $6.16 \mathrm{E}-21$ & $7.02 \mathrm{E}-20$ & FANCL \\
\hline ENSG00000185818 & -1.163457685 & $6.72 \mathrm{E}-21$ & $7.63 \mathrm{E}-20$ & NAT8L \\
\hline ENSG00000143942 & -0.728072397 & $6.72 \mathrm{E}-21$ & $7.63 \mathrm{E}-20$ & $\mathrm{CHAC} 2$ \\
\hline ENSG00000185862 & -0.776759935 & $1.13 \mathrm{E}-20$ & $1.27 \mathrm{E}-19$ & EVI2B \\
\hline ENSG00000164619 & -1.07660645 & $1.2 \mathrm{E}-20$ & $1.35 \mathrm{E}-19$ & BMPER \\
\hline ENSG00000058091 & -0.700546181 & $2.42 \mathrm{E}-20$ & $2.67 \mathrm{E}-19$ & CDK14 \\
\hline ENSG00000175556 & -0.6405596 & $2.9 \mathrm{E}-20$ & $3.19 \mathrm{E}-19$ & LONRF3 \\
\hline ENSG00000176641 & -0.725354894 & $2.93 \mathrm{E}-20$ & $3.22 \mathrm{E}-19$ & RNF152 \\
\hline ENSG00000135414 & -0.646867873 & $6.7 \mathrm{E}-20$ & $7.23 \mathrm{E}-19$ & GDF11 \\
\hline ENSG00000134569 & -0.789074753 & $6.71 \mathrm{E}-20$ & $7.24 \mathrm{E}-19$ & LRP4 \\
\hline ENSG00000080823 & -0.602706451 & $7.6 \mathrm{E}-20$ & $8.18 \mathrm{E}-19$ & MOK \\
\hline ENSG00000188312 & -0.722540228 & $8.34 \mathrm{E}-20$ & $8.96 \mathrm{E}-19$ & CENPP \\
\hline ENSG00000163568 & -0.962591724 & $1.46 \mathrm{E}-19$ & $1.56 \mathrm{E}-18$ & AIM2 \\
\hline ENSG00000160392 & -0.610014247 & $2.03 \mathrm{E}-19$ & $2.14 \mathrm{E}-18$ & C19orf47 \\
\hline ENSG00000119042 & -0.612708814 & $3.03 \mathrm{E}-19$ & $3.17 \mathrm{E}-18$ & SATB2 \\
\hline ENSG00000166532 & -0.673619231 & $3.77 \mathrm{E}-19$ & $3.91 \mathrm{E}-18$ & RIMKLB \\
\hline ENSG00000100483 & -0.677631261 & $8.66 \mathrm{E}-19$ & $8.8 \mathrm{E}-18$ & VCPKMT \\
\hline ENSG00000080493 & -1.327083425 & $2.48 \mathrm{E}-18$ & $2.44 \mathrm{E}-17$ & SLC4A4 \\
\hline ENSG00000248487 & -0.969369054 & $2.57 \mathrm{E}-18$ & $2.52 \mathrm{E}-17$ & ABHD14A \\
\hline ENSG00000153956 & -1.026853205 & $3.17 \mathrm{E}-18$ & $3.09 \mathrm{E}-17$ & CACNA2D1 \\
\hline ENSG00000204767 & -0.615679238 & $4.62 \mathrm{E}-18$ & $4.46 \mathrm{E}-17$ & FAM196B \\
\hline ENSG00000166582 & -0.670746514 & $5.34 \mathrm{E}-18$ & $5.15 \mathrm{E}-17$ & CENPV \\
\hline ENSG00000121578 & -0.646426771 & $5.55 \mathrm{E}-18$ & $5.33 \mathrm{E}-17$ & B4GALT4 \\
\hline ENSG00000175183 & -0.603690194 & $7.81 \mathrm{E}-18$ & $7.4 \mathrm{E}-17$ & CSRP2 \\
\hline ENSG00000134508 & -0.584140679 & $7.84 \mathrm{E}-18$ & $7.42 \mathrm{E}-17$ & CABLES1 \\
\hline
\end{tabular}


Table 3 (continued)

\begin{tabular}{|c|c|c|c|c|}
\hline Locus & $\log \mathrm{FC}$ & $P$-Value & FDR & Gene name \\
\hline ENSG00000154237 & -0.591563665 & $1.09 \mathrm{E}-17$ & $1.02 \mathrm{E}-16$ & LRRK1 \\
\hline ENSG00000118257 & -0.661860339 & $1.27 \mathrm{E}-17$ & $1.19 \mathrm{E}-16$ & NRP2 \\
\hline ENSG00000136160 & -0.728515383 & $1.31 \mathrm{E}-17$ & $1.22 \mathrm{E}-16$ & EDNRB \\
\hline ENSG00000172889 & -0.78025385 & $2.36 \mathrm{E}-17$ & $2.17 \mathrm{E}-16$ & EGFL7 \\
\hline ENSG00000170498 & -1.543019875 & $2.8 \mathrm{E}-17$ & $2.56 \mathrm{E}-16$ & KISS1 \\
\hline ENSG00000112394 & -1.707112548 & $4.7 \mathrm{E}-17$ & $4.24 \mathrm{E}-16$ & SLC16A10 \\
\hline ENSG00000151229 & -0.746387031 & $6.14 \mathrm{E}-17$ & $5.48 \mathrm{E}-16$ & SLC2A13 \\
\hline ENSG00000091129 & -1.696794072 & $6.83 \mathrm{E}-17$ & $6.05 \mathrm{E}-16$ & NRCAM \\
\hline ENSG00000171388 & -1.043380952 & $8.76 \mathrm{E}-17$ & $7.71 \mathrm{E}-16$ & APLN \\
\hline ENSG00000228300 & -0.706210371 & $9.96 \mathrm{E}-17$ & $8.73 \mathrm{E}-16$ & C19orf24 \\
\hline ENSG00000149970 & -0.855419499 & $1.04 \mathrm{E}-16$ & $9.09 \mathrm{E}-16$ & CNKSR2 \\
\hline ENSG00000162711 & -0.776561197 & $1.06 \mathrm{E}-16$ & $9.24 \mathrm{E}-16$ & NLRP3 \\
\hline ENSG00000156795 & -0.622341733 & $1.18 \mathrm{E}-16$ & $1.03 \mathrm{E}-15$ & WDYHV1 \\
\hline ENSG00000169684 & -0.74358858 & $2.58 \mathrm{E}-16$ & $2.21 \mathrm{E}-15$ & CHRNA5 \\
\hline ENSG00000197147 & -0.671838185 & $2.76 \mathrm{E}-16$ & $2.36 \mathrm{E}-15$ & LRRC8B \\
\hline ENSG00000206538 & -0.84083006 & $3.21 \mathrm{E}-16$ & $2.72 \mathrm{E}-15$ & VGLL3 \\
\hline ENSG00000133101 & -0.714667229 & $3.28 \mathrm{E}-16$ & $2.78 \mathrm{E}-15$ & CCNA1 \\
\hline ENSG00000107518 & -0.601853491 & $3.48 \mathrm{E}-16$ & $2.94 \mathrm{E}-15$ & ATRNL1 \\
\hline ENSG00000155495 & -1.359915587 & $4.29 \mathrm{E}-16$ & $3.61 \mathrm{E}-15$ & MAGEC1 \\
\hline ENSG00000181754 & -0.612040358 & $5.47 \mathrm{E}-16$ & $4.56 \mathrm{E}-15$ & AMIGO1 \\
\hline ENSG00000168685 & -0.629731176 & $6.4 \mathrm{E}-16$ & $5.31 \mathrm{E}-15$ & IL7R \\
\hline ENSG00000154274 & -0.654221576 & $6.52 \mathrm{E}-16$ & $5.41 \mathrm{E}-15$ & C4orf19 \\
\hline ENSG00000112425 & -0.870586941 & $6.91 \mathrm{E}-16$ & $5.72 \mathrm{E}-15$ & EPM2A \\
\hline ENSG00000128610 & -1.168896695 & $6.95 \mathrm{E}-16$ & $5.75 \mathrm{E}-15$ & FEZF1 \\
\hline ENSG00000154914 & -0.915019891 & $7.14 \mathrm{E}-16$ & $5.9 \mathrm{E}-15$ & USP43 \\
\hline ENSG00000082014 & -1.08393885 & $8.58 \mathrm{E}-16$ & $7.05 \mathrm{E}-15$ & SMARCD3 \\
\hline ENSG00000105929 & -0.876658986 & $3.48 \mathrm{E}-15$ & $2.74 \mathrm{E}-14$ & ATP6V0A4 \\
\hline ENSG00000169570 & -0.772019802 & $4.97 \mathrm{E}-15$ & $3.88 \mathrm{E}-14$ & DTWD2 \\
\hline ENSG00000131480 & -1.0578589 & $7.95 \mathrm{E}-15$ & $6.09 \mathrm{E}-14$ & AOC2 \\
\hline ENSG00000127311 & -0.757865784 & $8.99 \mathrm{E}-15$ & $6.87 \mathrm{E}-14$ & HELB \\
\hline ENSG00000169851 & -0.775603189 & $9.22 \mathrm{E}-15$ & $7.04 \mathrm{E}-14$ & PCDH7 \\
\hline ENSG00000026103 & -0.72906724 & $1.21 \mathrm{E}-14$ & $9.14 \mathrm{E}-14$ & FAS \\
\hline ENSG00000235194 & -0.79485048 & $3.52 \mathrm{E}-14$ & $2.56 \mathrm{E}-13$ & PPP1R3E \\
\hline ENSG00000105894 & -0.782561566 & $3.9 \mathrm{E}-14$ & $2.83 \mathrm{E}-13$ & PTN \\
\hline ENSG00000162545 & -1.145665693 & $4.19 \mathrm{E}-14$ & $3.04 \mathrm{E}-13$ & CAMK2N1 \\
\hline ENSG00000150764 & -0.704440404 & $4.83 \mathrm{E}-14$ & $3.49 \mathrm{E}-13$ & DIXDC1 \\
\hline ENSG00000018236 & -1.47880204 & $4.91 \mathrm{E}-14$ & $3.53 \mathrm{E}-13$ & CNTN1 \\
\hline ENSG00000187231 & -0.644113059 & $5.52 \mathrm{E}-14$ & $3.97 \mathrm{E}-13$ & SESTD1 \\
\hline ENSG00000110852 & -0.662960751 & $6.52 \mathrm{E}-14$ & $4.66 \mathrm{E}-13$ & CLEC2B \\
\hline ENSG00000170577 & -0.939095059 & $7.26 \mathrm{E}-14$ & $5.16 \mathrm{E}-13$ & SIX2 \\
\hline ENSG00000175893 & -0.614669715 & $1.1 \mathrm{E}-13$ & $7.72 \mathrm{E}-13$ & ZDHHC21 \\
\hline ENSG00000084734 & -1.185795317 & $1.34 \mathrm{E}-13$ & $9.33 \mathrm{E}-13$ & GCKR \\
\hline ENSG00000166689 & -0.674253903 & $1.62 \mathrm{E}-13$ & $1.13 \mathrm{E}-12$ & PLEKHA7 \\
\hline ENSG00000152669 & -0.704229096 & $1.78 \mathrm{E}-13$ & $1.23 \mathrm{E}-12$ & $\mathrm{CCNO}$ \\
\hline ENSG00000069667 & -0.817249087 & $1.88 \mathrm{E}-13$ & $1.3 \mathrm{E}-12$ & RORA \\
\hline ENSG00000167941 & -1.822789729 & $2.12 \mathrm{E}-13$ & $1.46 \mathrm{E}-12$ & SOST \\
\hline ENSG00000152270 & -0.627826609 & $2.26 \mathrm{E}-13$ & $1.55 \mathrm{E}-12$ & PDE3B \\
\hline
\end{tabular}


Table 3 (continued)

\begin{tabular}{|c|c|c|c|c|}
\hline Locus & $\log \mathrm{FC}$ & $P$-Value & FDR & Gene name \\
\hline ENSG00000152527 & -0.65848094 & $3.22 \mathrm{E}-13$ & $2.19 \mathrm{E}-12$ & PLEKHH2 \\
\hline ENSG00000070759 & -0.583708292 & $4.71 \mathrm{E}-13$ & $3.16 \mathrm{E}-12$ & TESK2 \\
\hline ENSG00000068615 & -0.935596963 & $4.71 \mathrm{E}-13$ & $3.16 \mathrm{E}-12$ & REEP1 \\
\hline ENSG00000163328 & -1.081666267 & $5.61 \mathrm{E}-13$ & $3.75 \mathrm{E}-12$ & GPR155 \\
\hline ENSG00000005249 & -0.989574304 & $7.85 \mathrm{E}-13$ & $5.19 \mathrm{E}-12$ & PRKAR2B \\
\hline ENSG00000120279 & -1.086387629 & $8.3 \mathrm{E}-13$ & $5.48 \mathrm{E}-12$ & MYCT1 \\
\hline ENSG00000140450 & -0.633642782 & $8.3 \mathrm{E}-13$ & $5.48 \mathrm{E}-12$ & ARRDC4 \\
\hline ENSG00000197415 & -0.649632825 & $1.08 \mathrm{E}-12$ & $7.02 \mathrm{E}-12$ & VEPH1 \\
\hline ENSG00000170417 & -0.93939747 & $1.48 \mathrm{E}-12$ & $9.5 \mathrm{E}-12$ & TMEM182 \\
\hline ENSG00000126803 & -1.11469054 & $1.54 \mathrm{E}-12$ & $9.92 \mathrm{E}-12$ & HSPA2 \\
\hline ENSG00000170899 & -0.684484123 & $1.7 \mathrm{E}-12$ & $1.09 \mathrm{E}-11$ & GSTA4 \\
\hline ENSG00000168077 & -1.165454147 & $2.15 \mathrm{E}-12$ & $1.37 \mathrm{E}-11$ & SCARA3 \\
\hline ENSG00000181418 & -1.58931145 & $2.26 \mathrm{E}-12$ & $1.44 \mathrm{E}-11$ & DDN \\
\hline ENSG00000154040 & -0.938234977 & $2.4 \mathrm{E}-12$ & $1.52 \mathrm{E}-11$ & CABYR \\
\hline ENSG00000188060 & -1.258637511 & $2.57 \mathrm{E}-12$ & $1.62 \mathrm{E}-11$ & RAB42 \\
\hline ENSG00000108852 & -0.843142689 & $2.7 \mathrm{E}-12$ & $1.71 \mathrm{E}-11$ & MPP2 \\
\hline ENSG00000166823 & -0.986678684 & $4.18 \mathrm{E}-12$ & $2.61 \mathrm{E}-11$ & MESP1 \\
\hline ENSG00000104154 & -0.597478232 & $4.45 \mathrm{E}-12$ & $2.77 \mathrm{E}-11$ & SLC30A4 \\
\hline ENSG00000106025 & -0.593643936 & $6.72 \mathrm{E}-12$ & $4.1 \mathrm{E}-11$ & TSPAN12 \\
\hline ENSG00000163472 & -0.745176085 & $1.25 \mathrm{E}-11$ & $7.49 \mathrm{E}-11$ & TMEM79 \\
\hline ENSG00000178343 & -0.948648227 & $1.45 \mathrm{E}-11$ & $8.61 \mathrm{E}-11$ & SHISA3 \\
\hline ENSG00000140044 & -0.769542054 & $1.64 \mathrm{E}-11$ & $9.7 \mathrm{E}-11$ & JDP2 \\
\hline ENSG00000166813 & -0.590817747 & $1.72 \mathrm{E}-11$ & $1.01 \mathrm{E}-10$ & KIF7 \\
\hline ENSG00000143382 & -1.022866716 & $1.85 \mathrm{E}-11$ & $1.09 \mathrm{E}-10$ & ADAMTSL4 \\
\hline ENSG00000280670 & -0.649067763 & $2.08 \mathrm{E}-11$ & $1.22 \mathrm{E}-10$ & CCDC163 \\
\hline ENSG00000143320 & -0.873020398 & $2.19 \mathrm{E}-11$ & $1.28 \mathrm{E}-10$ & CRABP2 \\
\hline ENSG00000090020 & -0.695130894 & $2.54 \mathrm{E}-11$ & $1.47 \mathrm{E}-10$ & SLC9A1 \\
\hline ENSG00000116141 & -0.722567149 & $2.54 \mathrm{E}-11$ & $1.48 \mathrm{E}-10$ & MARK1 \\
\hline ENSG00000165655 & -0.867909098 & $2.67 \mathrm{E}-11$ & $1.54 \mathrm{E}-10$ & ZNF503 \\
\hline ENSG00000128694 & -0.624482372 & $2.9 \mathrm{E}-11$ & $1.67 \mathrm{E}-10$ & OSGEPL1 \\
\hline ENSG00000135378 & -0.665705024 & $4.18 \mathrm{E}-11$ & $2.39 \mathrm{E}-10$ & PRRG4 \\
\hline ENSG00000135333 & -1.203520059 & $4.22 \mathrm{E}-11$ & $2.42 \mathrm{E}-10$ & EPHA7 \\
\hline ENSG00000008277 & -0.724949048 & $4.27 \mathrm{E}-11$ & $2.44 \mathrm{E}-10$ & ADAM22 \\
\hline ENSG00000134321 & -0.688580763 & $4.43 \mathrm{E}-11$ & $2.53 \mathrm{E}-10$ & RSAD2 \\
\hline ENSG00000196843 & -0.607550863 & $4.54 \mathrm{E}-11$ & $2.59 \mathrm{E}-10$ & ARID5A \\
\hline ENSG00000186205 & -0.637588673 & $4.66 \mathrm{E}-11$ & $2.65 \mathrm{E}-10$ & MARC1 \\
\hline ENSG00000171365 & -0.672055612 & $5.69 \mathrm{E}-11$ & $3.21 \mathrm{E}-10$ & CLCN5 \\
\hline ENSG00000128872 & -0.584078549 & $5.78 \mathrm{E}-11$ & $3.26 \mathrm{E}-10$ & TMOD2 \\
\hline ENSG00000221890 & -0.782195142 & $6.9 \mathrm{E}-11$ & $3.84 \mathrm{E}-10$ & NPTXR \\
\hline ENSG00000234602 & -0.995961314 & $7.14 \mathrm{E}-11$ & $3.97 \mathrm{E}-10$ & MCIDAS \\
\hline ENSG00000157303 & -0.96944952 & $7.23 \mathrm{E}-11$ & $4.02 \mathrm{E}-10$ & SUSD3 \\
\hline ENSG00000164414 & -0.591096299 & $7.69 \mathrm{E}-11$ & $4.27 \mathrm{E}-10$ & SLC35A1 \\
\hline ENSG00000178401 & -0.94668282 & $7.84 \mathrm{E}-11$ & $4.35 \mathrm{E}-10$ & DNAJC22 \\
\hline ENSG00000139973 & -0.875379862 & $8.35 \mathrm{E}-11$ & $4.61 \mathrm{E}-10$ & SYT16 \\
\hline ENSG00000182118 & -0.593515976 & $8.35 \mathrm{E}-11$ & $4.61 \mathrm{E}-10$ & FAM89A \\
\hline ENSG00000184254 & -0.62334185 & $8.41 \mathrm{E}-11$ & $4.64 \mathrm{E}-10$ & ALDH1A3 \\
\hline ENSG00000130244 & -0.638701689 & $9.48 \mathrm{E}-11$ & $5.2 \mathrm{E}-10$ & FAM98C \\
\hline
\end{tabular}


Table 3 (continued)

\begin{tabular}{|c|c|c|c|c|}
\hline Locus & $\log \mathrm{FC}$ & $P$-Value & FDR & Gene name \\
\hline ENSG00000011332 & -0.682979301 & $1.07 \mathrm{E}-10$ & $5.86 \mathrm{E}-10$ & DPF1 \\
\hline ENSG00000151468 & -0.953297034 & $1.3 \mathrm{E}-10$ & $7.06 \mathrm{E}-10$ & $\mathrm{CCDC} 3$ \\
\hline ENSG00000162999 & -1.755455161 & $1.6 \mathrm{E}-10$ & $8.58 \mathrm{E}-10$ & DUSP19 \\
\hline ENSG00000116396 & -0.896224276 & $1.67 \mathrm{E}-10$ & $8.97 \mathrm{E}-10$ & $\mathrm{KCNC4}$ \\
\hline ENSG00000105290 & -0.98909701 & $1.99 \mathrm{E}-10$ & $1.06 \mathrm{E}-09$ & APLP1 \\
\hline ENSG00000184838 & -0.709702383 & $2.05 \mathrm{E}-10$ & $1.09 \mathrm{E}-09$ & PRR16 \\
\hline ENSG00000131471 & -1.508965698 & $2.07 \mathrm{E}-10$ & $1.1 \mathrm{E}-09$ & AOC3 \\
\hline ENSG00000141404 & -1.039495113 & $2.15 \mathrm{E}-10$ & $1.14 \mathrm{E}-09$ & GNAL \\
\hline ENSG00000125384 & -1.217017506 & $2.82 \mathrm{E}-10$ & $1.47 \mathrm{E}-09$ & PTGER2 \\
\hline ENSG00000061918 & -0.775981343 & $3.62 \mathrm{E}-10$ & $1.87 \mathrm{E}-09$ & GUCY1B3 \\
\hline ENSG00000156172 & -0.844143968 & $3.84 \mathrm{E}-10$ & $1.98 \mathrm{E}-09$ & C8orf37 \\
\hline ENSG00000100092 & -0.612795457 & $5.7 \mathrm{E}-10$ & $2.89 \mathrm{E}-09$ & SH3BP1 \\
\hline ENSG00000175175 & -0.668290059 & $6.19 \mathrm{E}-10$ & $3.13 \mathrm{E}-09$ & PPM1E \\
\hline ENSG00000150893 & -0.822911314 & $7.32 \mathrm{E}-10$ & $3.67 \mathrm{E}-09$ & FREM2 \\
\hline ENSG00000173890 & -0.725043577 & $9.66 \mathrm{E}-10$ & $4.79 \mathrm{E}-09$ & GPR160 \\
\hline ENSG00000129595 & -0.756168514 & $1.32 \mathrm{E}-09$ & $6.44 \mathrm{E}-09$ & EPB41L4A \\
\hline ENSG00000180739 & -1.212317922 & $1.43 \mathrm{E}-09$ & 0.000000007 & S1PR5 \\
\hline ENSG00000104369 & -0.713893389 & $1.49 \mathrm{E}-09$ & $7.28 \mathrm{E}-09$ & JPH1 \\
\hline ENSG00000163884 & -1.001721809 & $2.22 \mathrm{E}-09$ & $1.06 \mathrm{E}-08$ & KLF15 \\
\hline ENSG00000172543 & -1.443971336 & $2.6 \mathrm{E}-09$ & $1.23 \mathrm{E}-08$ & CTSW \\
\hline ENSG00000034533 & -0.602893943 & $2.98 \mathrm{E}-09$ & $1.41 \mathrm{E}-08$ & ASTE1 \\
\hline ENSG00000016391 & -1.454323229 & 0.000000003 & $1.42 \mathrm{E}-08$ & $\mathrm{CHDH}$ \\
\hline ENSG00000163412 & -1.455095924 & $3.22 \mathrm{E}-09$ & $1.51 \mathrm{E}-08$ & EIF4E3 \\
\hline ENSG00000130821 & -0.69419495 & $3.28 \mathrm{E}-09$ & $1.54 \mathrm{E}-08$ & SLC6A8 \\
\hline ENSG00000135929 & -0.792267569 & $3.68 \mathrm{E}-09$ & $1.72 \mathrm{E}-08$ & CYP27A1 \\
\hline ENSG00000174370 & -1.005785086 & $4.1 \mathrm{E}-09$ & $1.91 \mathrm{E}-08$ & C11orf 45 \\
\hline ENSG00000025423 & -0.998326574 & $4.63 \mathrm{E}-09$ & $2.14 \mathrm{E}-08$ & HSD17B6 \\
\hline ENSG00000111199 & -1.268393502 & $5.71 \mathrm{E}-09$ & $2.62 \mathrm{E}-08$ & TRPV4 \\
\hline ENSG00000155974 & -0.67183537 & $6.04 \mathrm{E}-09$ & $2.76 \mathrm{E}-08$ & GRIP1 \\
\hline ENSG00000147606 & -1.027590206 & $6.16 \mathrm{E}-09$ & $2.81 \mathrm{E}-08$ & SLC26A7 \\
\hline ENSG00000065675 & -1.501715725 & $6.81 \mathrm{E}-09$ & $3.09 \mathrm{E}-08$ & PRKCQ \\
\hline ENSG00000179841 & -0.749380697 & $7.31 \mathrm{E}-09$ & $3.31 \mathrm{E}-08$ & AKAP5 \\
\hline ENSG00000260822 & -0.582080039 & 0.000000227 & 0.000000864 & AC004656.1 \\
\hline ENSG00000156265 & -0.79220517 & $8.14 \mathrm{E}-09$ & $3.67 \mathrm{E}-08$ & MAP3K7CL \\
\hline ENSG00000079215 & -0.679149885 & $9.51 \mathrm{E}-09$ & $4.25 \mathrm{E}-08$ & SLC1A3 \\
\hline ENSG00000221944 & -0.600202219 & $9.68 \mathrm{E}-09$ & $4.32 \mathrm{E}-08$ & TIGD1 \\
\hline ENSG00000101680 & -0.905321065 & $1.11 \mathrm{E}-08$ & $4.94 \mathrm{E}-08$ & LAMA1 \\
\hline ENSG00000126785 & -0.618262682 & $1.15 \mathrm{E}-08$ & $5.08 \mathrm{E}-08$ & RHOJ \\
\hline ENSG00000205628 & -0.582080325 & 0.000251743 & 0.000637414 & LINC01446 \\
\hline ENSG00000223773 & -0.583523523 & 0.008232673 & 0.015658057 & CD99P1 \\
\hline ENSG00000282826 & -0.583636435 & 0.008093028 & 0.015414788 & FRG1CP \\
\hline ENSG00000241544 & -0.586635592 & 0.001586262 & 0.0034698 & LINC02029 \\
\hline ENSG00000122420 & -0.7597314 & $1.73 \mathrm{E}-08$ & $7.48 \mathrm{E}-08$ & PTGFR \\
\hline ENSG00000111879 & -0.681429858 & $1.79 \mathrm{E}-08$ & $7.72 \mathrm{E}-08$ & FAM184A \\
\hline ENSG00000186162 & -0.589358402 & $4.94 \mathrm{E}-16$ & $4.13 \mathrm{E}-15$ & CIDECP \\
\hline ENSG00000225793 & -0.598603858 & 0.002182824 & 0.004653786 & AL080250.1 \\
\hline ENSG00000164674 & -0.834186124 & $2.35 \mathrm{E}-08$ & 0.0000001 & SYTL3 \\
\hline
\end{tabular}


Table 3 (continued)

\begin{tabular}{|c|c|c|c|c|}
\hline Locus & $\log \mathrm{FC}$ & $P$-Value & FDR & Gene name \\
\hline ENSG00000163710 & -0.74510529 & $2.55 \mathrm{E}-08$ & 0.000000108 & PCOLCE2 \\
\hline ENSG00000138741 & -1.124387768 & $2.62 \mathrm{E}-08$ & 0.000000111 & TRPC3 \\
\hline ENSG00000147003 & -0.945694847 & $2.92 \mathrm{E}-08$ & 0.000000123 & TMEM27 \\
\hline ENSG00000168874 & -1.171454266 & $3.51 \mathrm{E}-08$ & 0.000000147 & ATOH8 \\
\hline ENSG00000100077 & -0.645354414 & $4.29 \mathrm{E}-08$ & 0.000000178 & GRK3 \\
\hline ENSG00000206652 & -0.600319045 & 0.0041797 & 0.008468391 & RNU1-1 \\
\hline ENSG00000056998 & -1.159597278 & $4.96 \mathrm{E}-08$ & 0.000000204 & GYG2 \\
\hline ENSG00000110811 & -1.364088188 & $5.18 \mathrm{E}-08$ & 0.000000212 & $\mathrm{P} 3 \mathrm{H} 3$ \\
\hline ENSG00000234155 & -0.603589219 & 0.0000182 & 0.0000545 & AL135903.2 \\
\hline ENSG00000132718 & -0.675745243 & $5.96 \mathrm{E}-08$ & 0.000000243 & SYT11 \\
\hline ENSG00000262202 & -0.611008242 & 0.00012338 & 0.000327359 & AC007952.4 \\
\hline ENSG00000263535 & -0.612042556 & 0.004466662 & 0.009001187 & AC134669.1 \\
\hline ENSG00000260966 & -0.619021953 & 0.000673059 & 0.001578768 & AP001486.2 \\
\hline ENSG00000100321 & -1.052508227 & 0.000000103 & 0.000000411 & SYNGR1 \\
\hline ENSG00000201558 & -0.622105346 & $6.01 \mathrm{E}-11$ & $3.37 \mathrm{E}-10$ & RNVU1-6 \\
\hline ENSG00000139173 & -1.036479375 & 0.000000114 & 0.00000045 & TMEM117 \\
\hline ENSG00000273320 & -0.627836339 & 0.001733515 & 0.003763066 & AC007032.1 \\
\hline ENSG00000175764 & -0.612825531 & 0.000000149 & 0.000000581 & TTLL11 \\
\hline ENSG00000196405 & -0.658711721 & 0.000000158 & 0.000000615 & EVL \\
\hline ENSG00000169085 & -0.59715125 & 0.000000164 & 0.000000633 & C8orf46 \\
\hline ENSG00000255389 & -0.628197788 & 0.004805773 & 0.009610814 & Z97989.1 \\
\hline ENSG00000272821 & -0.628905849 & 0.00000876 & 0.0000274 & U62317.3 \\
\hline ENSG00000222365 & -0.632247461 & 0.00000032 & 0.0000012 & SNORD12B \\
\hline ENSG00000245146 & -0.63578959 & 0.002320634 & 0.004924648 & LINC01024 \\
\hline ENSG00000184271 & -0.985548652 & 0.000000242 & 0.000000921 & POU6F1 \\
\hline ENSG00000027075 & -0.603546465 & 0.000000268 & 0.00000101 & PRKCH \\
\hline ENSG00000249456 & -0.640283418 & 0.002467147 & 0.00521089 & AL731577.2 \\
\hline ENSG00000159263 & -0.785381395 & 0.000000284 & 0.00000107 & SIM2 \\
\hline ENSG00000268001 & -0.641308425 & 0.000000459 & 0.00000168 & CARD8-AS1 \\
\hline ENSG00000272779 & -0.642724602 & $3.69 \mathrm{E}-15$ & $2.9 \mathrm{E}-14$ & AC245060.4 \\
\hline ENSG00000261175 & -0.644864636 & 0.001089512 & 0.00245603 & LINC02188 \\
\hline ENSG00000259959 & -0.649511362 & 0.00000882 & 0.0000276 & AC107068.1 \\
\hline ENSG00000204228 & -0.962404554 & 0.000000335 & 0.00000125 & HSD17B8 \\
\hline ENSG00000114270 & -0.589453951 & 0.00000035 & 0.0000013 & COL7A1 \\
\hline ENSG00000226887 & -1.126238941 & 0.000000395 & 0.00000146 & ERVMER34-1 \\
\hline ENSG00000117425 & -0.660152077 & 0.000000436 & 0.0000016 & PTCH2 \\
\hline ENSG00000250299 & -0.650539806 & 0.000742332 & 0.001725842 & MRPS31P4 \\
\hline ENSG00000055732 & -0.706315288 & 0.000000475 & 0.00000174 & MCOLN3 \\
\hline ENSG00000233382 & -0.654372192 & 0.001896591 & 0.004089349 & NKAPP1 \\
\hline ENSG00000175745 & -0.605230114 & 0.000000521 & 0.0000019 & NR2F1 \\
\hline ENSG00000232093 & -0.658713398 & 0.004308088 & 0.008706828 & DCST1-AS1 \\
\hline ENSG00000165716 & -0.84644461 & 0.000000701 & 0.00000252 & FAM69B \\
\hline ENSG00000131187 & -0.649231899 & 0.00000073 & 0.00000261 & $\mathrm{~F} 12$ \\
\hline ENSG00000170775 & -0.603129994 & 0.000000804 & 0.00000286 & GPR37 \\
\hline ENSG00000164749 & -0.714196763 & 0.000000822 & 0.00000292 & HNF4G \\
\hline ENSG00000256663 & -0.661542619 & 0.00000329 & 0.0000109 & AC112777.1 \\
\hline ENSG00000228742 & -0.662633295 & 0.000530017 & 0.001268157 & AC002384.1 \\
\hline
\end{tabular}


Table 3 (continued)

\begin{tabular}{|c|c|c|c|c|}
\hline Locus & $\log \mathrm{FC}$ & $P$-Value & FDR & Gene name \\
\hline ENSG00000198515 & -1.13484411 & 0.00000108 & 0.00000381 & CNGA1 \\
\hline ENSG00000127324 & -0.591605084 & 0.00000113 & 0.00000397 & TSPAN8 \\
\hline ENSG00000237870 & -0.666779213 & $5.37 \mathrm{E}-08$ & 0.00000022 & AC073130.1 \\
\hline ENSG00000145040 & -0.999709672 & 0.00000138 & 0.0000048 & $\mathrm{UCN} 2$ \\
\hline ENSG00000197959 & -0.728336114 & 0.00000158 & 0.00000543 & DNM3 \\
\hline ENSG00000237187 & -0.673528302 & $1.09 \mathrm{E}-14$ & $8.29 \mathrm{E}-14$ & NR2F1-AS1 \\
\hline ENSG00000158050 & -0.888282466 & 0.00000182 & 0.0000062 & DUSP2 \\
\hline ENSG00000272269 & -0.674471909 & 0.000000168 & 0.000000648 & AL138724.1 \\
\hline ENSG00000162733 & -0.85420253 & 0.00000199 & 0.00000675 & DDR2 \\
\hline ENSG00000170458 & -0.753322548 & 0.00000203 & 0.00000689 & CD14 \\
\hline ENSG00000275131 & -0.675077093 & $8.09 \mathrm{E}-09$ & $3.65 \mathrm{E}-08$ & AC241952.1 \\
\hline ENSG00000246228 & -0.676059031 & $3.1 \mathrm{E}-20$ & $3.4 \mathrm{E}-19$ & CASC8 \\
\hline ENSG00000254887 & -0.676505955 & 0.001307319 & 0.002905128 & AC010247.1 \\
\hline ENSG00000230445 & -0.683831123 & 0.0000288 & 0.0000842 & LRRC37A6P \\
\hline ENSG00000204335 & -0.655622518 & 0.00000381 & 0.0000125 & SP5 \\
\hline ENSG00000121207 & -1.100166986 & 0.00000424 & 0.0000138 & LRAT \\
\hline ENSG00000151687 & -0.657707604 & 0.00000479 & 0.0000156 & ANKAR \\
\hline ENSG00000153162 & -0.713261029 & 0.00000516 & 0.0000166 & BMP6 \\
\hline ENSG00000120693 & -0.923295877 & 0.0000056 & 0.000018 & SMAD9 \\
\hline ENSG00000169330 & -0.583313432 & 0.00000629 & 0.0000201 & KIAA1024 \\
\hline ENSG00000250731 & -0.68687386 & 0.000258139 & 0.000651726 & ТРМ3Р6 \\
\hline ENSG00000130675 & -0.922679936 & 0.00000653 & 0.0000208 & MNX1 \\
\hline ENSG00000117318 & -0.846439456 & 0.00000656 & 0.0000209 & ID3 \\
\hline ENSG00000206052 & -0.91285047 & 0.00000696 & 0.0000221 & DOK6 \\
\hline ENSG00000119699 & -0.79719179 & 0.00000704 & 0.0000223 & TGFB3 \\
\hline ENSG00000100867 & -0.695196402 & 0.00000731 & 0.0000231 & DHRS2 \\
\hline ENSG00000244486 & -0.991783568 & 0.00000738 & 0.0000233 & SCARF2 \\
\hline ENSG00000166963 & -1.102639672 & 0.00000789 & 0.0000249 & MAP1A \\
\hline ENSG00000254615 & -0.690954391 & $9.46 \mathrm{E}-10$ & $4.7 \mathrm{E}-09$ & AC027031.2 \\
\hline ENSG00000163009 & -0.691160202 & 0.000483706 & 0.0011635 & C2orf48 \\
\hline ENSG00000109705 & -0.700668566 & 0.00000911 & 0.0000284 & NKX3-2 \\
\hline ENSG00000178662 & -0.707748281 & 0.00000999 & 0.0000311 & CSRNP3 \\
\hline ENSG00000248121 & -0.691464153 & 0.001506607 & 0.003312113 & SMURF2P1 \\
\hline ENSG00000254893 & -0.826566134 & 0.0000103 & 0.0000321 & AC113404.3 \\
\hline ENSG00000164841 & -0.622993759 & 0.0000104 & 0.0000323 & TMEM74 \\
\hline ENSG00000215210 & -0.702924697 & 0.001207828 & 0.002698653 & RBMXP2 \\
\hline ENSG00000267383 & -0.703518554 & 0.0000101 & 0.0000314 & AC011447.3 \\
\hline ENSG00000277072 & -0.70804624 & 0.000396301 & 0.000965996 & STAG3L2 \\
\hline ENSG00000111012 & -1.073265175 & 0.0000114 & 0.000035 & CYP27B1 \\
\hline ENSG00000147036 & -0.759385987 & 0.0000114 & 0.000035 & LANCL3 \\
\hline ENSG00000186019 & -0.7083644 & 0.000754381 & 0.001750497 & AC021092.1 \\
\hline ENSG00000259673 & -0.709890208 & $6.48 \mathrm{E}-10$ & $3.27 \mathrm{E}-09$ & IQCH-AS1 \\
\hline ENSG00000258429 & -0.624426456 & 0.0000121 & 0.0000371 & PDF \\
\hline ENSG00000141391 & -0.60827287 & 0.0000122 & 0.0000373 & PRELID3A \\
\hline ENSG00000211448 & -0.972541619 & 0.0000124 & 0.0000381 & $\mathrm{DIO} 2$ \\
\hline ENSG00000115616 & -0.665234335 & 0.0000133 & 0.0000408 & SLC9A2 \\
\hline ENSG00000112599 & -0.735375549 & 0.0000157 & 0.0000474 & GUCA1B \\
\hline
\end{tabular}


Table 3 (continued)

\begin{tabular}{|c|c|c|c|c|}
\hline Locus & $\log \mathrm{FC}$ & $P$-Value & FDR & Gene name \\
\hline ENSG00000204792 & -0.712824082 & 0.00000285 & 0.00000946 & LINC01291 \\
\hline ENSG00000170629 & -0.718917939 & $7.44 \mathrm{E}-08$ & 0.0000003 & DPY19L2P2 \\
\hline ENSG00000270607 & -0.721426273 & 0.0000854 & 0.000231989 & AC009549.1 \\
\hline ENSG00000166415 & -0.915490775 & 0.0000193 & 0.0000577 & WDR72 \\
\hline ENSG00000188985 & -0.721464876 & 0.000000975 & 0.00000344 & DHFRP1 \\
\hline ENSG00000270696 & -0.72171295 & $5.63 \mathrm{E}-13$ & $3.76 \mathrm{E}-12$ & AC005034.3 \\
\hline ENSG00000261087 & -0.722660516 & 0.000910157 & 0.00208174 & AP003469.4 \\
\hline ENSG00000224167 & -0.72658689 & $1.62 \mathrm{E}-08$ & $7.06 \mathrm{E}-08$ & AL390729.1 \\
\hline ENSG00000115738 & -0.875366803 & 0.0000248 & 0.0000733 & ID2 \\
\hline ENSG00000007968 & -0.997190263 & 0.0000264 & 0.0000779 & $\mathrm{E} 2 \mathrm{~F} 2$ \\
\hline ENSG00000133687 & -0.885164289 & 0.0000268 & 0.000079 & TMTC1 \\
\hline ENSG00000260442 & -0.727912721 & 0.000664516 & 0.001559564 & ATP2A1-AS1 \\
\hline ENSG00000178184 & -0.791109251 & 0.0000279 & 0.0000819 & PARD6G \\
\hline ENSG00000276672 & -0.733771157 & $3.09 \mathrm{E}-18$ & $3.03 \mathrm{E}-17$ & AL161891.1 \\
\hline ENSG00000177465 & -0.817978872 & 0.0000291 & 0.0000851 & ACOT4 \\
\hline ENSG00000264350 & -0.736318413 & 0.0000175 & 0.0000525 & AC090897.1 \\
\hline ENSG00000238142 & -0.738440741 & 0.00083095 & 0.001913575 & BX284668.5 \\
\hline ENSG00000143028 & -0.730875189 & 0.0000305 & 0.0000888 & SYPL2 \\
\hline ENSG00000173320 & -0.595801591 & 0.000032 & 0.0000929 & STOX2 \\
\hline ENSG00000260000 & -0.738881279 & 0.0000167 & 0.0000505 & AL133338.1 \\
\hline ENSG00000177822 & -0.741978242 & 0.00003 & 0.0000875 & AC098864.1 \\
\hline ENSG00000156140 & -0.669690954 & 0.0000439 & 0.000124705 & ADAMTS3 \\
\hline ENSG00000241360 & -0.647292344 & 0.0000467 & 0.000132129 & PDXP \\
\hline ENSG00000149646 & -0.661096929 & 0.0000498 & 0.000140129 & CNBD2 \\
\hline ENSG00000164920 & -0.58797654 & 0.0000546 & 0.000152834 & OSR2 \\
\hline ENSG00000273033 & -0.764755287 & $1.97 \mathrm{E}-08$ & $8.47 \mathrm{E}-08$ & LINC02035 \\
\hline ENSG00000116819 & -0.594037737 & 0.0000587 & 0.000163798 & TFAP2E \\
\hline ENSG00000274213 & -0.767601803 & 0.000644991 & 0.001516995 & AC015912.3 \\
\hline ENSG00000240929 & -0.770831237 & $1.19 \mathrm{E}-19$ & $1.28 \mathrm{E}-18$ & HIST2H2BB \\
\hline ENSG00000184005 & -0.872567649 & 0.0000797 & 0.000218143 & ST6GALNAC3 \\
\hline ENSG00000247556 & -0.7791386 & $8.2 \mathrm{E}-70$ & $6.74 \mathrm{E}-68$ & OIP5-AS1 \\
\hline ENSG00000016402 & -0.767097162 & 0.0000806 & 0.000220457 & IL20RA \\
\hline ENSG00000140465 & -0.704109542 & 0.000082 & 0.000223701 & CYP1A1 \\
\hline ENSG00000240024 & -0.786762956 & $3.5 \mathrm{E}-29$ & $6.14 \mathrm{E}-28$ & LINC00888 \\
\hline ENSG00000128596 & -0.690766509 & 0.0000891 & 0.000241372 & $\mathrm{CCDC} 136$ \\
\hline ENSG00000176125 & -0.855443147 & 0.0000949 & 0.00025624 & UFSP1 \\
\hline ENSG00000165730 & -0.595042604 & 0.0000969 & 0.000261295 & STOX1 \\
\hline ENSG00000119915 & -0.779823964 & 0.000105488 & 0.00028257 & ELOVL3 \\
\hline ENSG00000251350 & -0.79220264 & 0.0000027 & 0.00000897 & LINC02475 \\
\hline ENSG00000177181 & -0.894622588 & 0.000124158 & 0.00032929 & RIMKLA \\
\hline ENSG00000280079 & -0.80571522 & $5.25 \mathrm{E}-09$ & $2.41 \mathrm{E}-08$ & AC011447.7 \\
\hline ENSG00000279059 & -0.827433939 & 0.0000225 & 0.000067 & AC007485.2 \\
\hline ENSG00000184486 & -0.606547058 & 0.000159816 & 0.000418622 & POU3F2 \\
\hline ENSG00000138395 & -0.702091541 & 0.00016143 & 0.000422511 & CDK15 \\
\hline ENSG00000095637 & -0.610468574 & 0.000162344 & 0.000424481 & SORBS1 \\
\hline ENSG00000275185 & -0.828054896 & 0.00000027 & 0.00000102 & AC130324.3 \\
\hline ENSG00000135643 & -0.61786301 & 0.000180308 & 0.000468496 & KCNMB4 \\
\hline
\end{tabular}


Table 3 (continued)

\begin{tabular}{|c|c|c|c|c|}
\hline Locus & $\log \mathrm{FC}$ & $P$-Value & FDR & Gene name \\
\hline ENSG00000205002 & -0.777190629 & 0.000186764 & 0.000483708 & AARD \\
\hline ENSG00000229368 & -0.82873561 & 0.0000576 & 0.000160817 & AC090587.2 \\
\hline ENSG00000123096 & -0.61800128 & 0.00020928 & 0.000537779 & SSPN \\
\hline ENSG00000160219 & -0.713815817 & 0.000226571 & 0.000579039 & GAB3 \\
\hline ENSG00000119737 & -0.654203266 & 0.00024644 & 0.000625436 & GPR75 \\
\hline ENSG00000263272 & -0.832845527 & 0.0000107 & 0.0000332 & AC004148.2 \\
\hline ENSG00000204789 & -0.836003991 & $1.67 \mathrm{E}-10$ & $8.96 \mathrm{E}-10$ & ZNF204P \\
\hline ENSG00000279207 & -0.839738829 & 0.00000227 & 0.00000765 & AC015813.6 \\
\hline ENSG00000278291 & -0.844858432 & 0.000507367 & 0.001217292 & AL161772.1 \\
\hline ENSG00000233117 & -0.857807409 & $2.24 \mathrm{E}-13$ & $1.54 \mathrm{E}-12$ & LINC00702 \\
\hline ENSG00000164778 & -0.858184757 & 0.000294259 & 0.000735145 & EN2 \\
\hline ENSG00000173376 & -0.799990294 & 0.000322836 & 0.000799238 & NDNF \\
\hline ENSG00000186340 & -0.612224671 & 0.000327986 & 0.000810917 & THBS2 \\
\hline ENSG00000226608 & -0.86563425 & $2.31 \mathrm{E}-08$ & $9.86 \mathrm{E}-08$ & FTLP3 \\
\hline ENSG00000150510 & -0.635813794 & 0.000354515 & 0.000870281 & FAM124A \\
\hline ENSG00000129167 & -0.767846935 & 0.000362165 & 0.000887408 & TPH1 \\
\hline ENSG00000255224 & -0.871481211 & 0.000139626 & 0.000368601 & AC109322.1 \\
\hline ENSG00000132932 & -0.844803117 & 0.000408508 & 0.000992987 & ATP8A2 \\
\hline ENSG00000179546 & -0.62615245 & 0.000418009 & 0.001014768 & HTR1D \\
\hline ENSG00000124772 & -0.758950016 & 0.000448513 & 0.001084408 & CPNE5 \\
\hline ENSG00000132016 & -0.595302993 & 0.000460125 & 0.001110729 & C19orf57 \\
\hline ENSG00000227908 & -0.87629061 & 0.0000218 & 0.0000647 & FLJ31104 \\
\hline ENSG00000272462 & -0.889371251 & 0.000000306 & 0.00000115 & U91328.2 \\
\hline ENSG00000232368 & -0.891946046 & $8.72 \mathrm{E}-10$ & $4.34 \mathrm{E}-09$ & FTLP2 \\
\hline ENSG00000274020 & -0.902527943 & 0.000000219 & 0.000000837 & LINC01138 \\
\hline ENSG00000167705 & -0.656826415 & 0.00055993 & 0.001333171 & RILP \\
\hline ENSG00000163596 & -0.716182102 & 0.000585479 & 0.001386711 & ICA1L \\
\hline ENSG00000162601 & -0.652501753 & 0.000599957 & 0.0014187 & MYSM1 \\
\hline ENSG00000250132 & -0.906972213 & 0.000126211 & 0.000334668 & AC004803.1 \\
\hline ENSG00000143494 & -0.629613078 & 0.000659815 & 0.001549361 & VASH2 \\
\hline ENSG00000276107 & -0.914373714 & $3.98 \mathrm{E}-09$ & $1.85 \mathrm{E}-08$ & AC037198.2 \\
\hline ENSG00000238266 & -0.920917248 & $2.39 \mathrm{E}-11$ & $1.39 \mathrm{E}-10$ & LINC00707 \\
\hline ENSG00000128203 & -0.656798742 & 0.000682964 & 0.001599429 & ASPHD2 \\
\hline ENSG00000187391 & -0.775390286 & 0.000695998 & 0.001627632 & MAGI2 \\
\hline ENSG00000198157 & -0.672184208 & 0.000719226 & 0.001676278 & HMGN5 \\
\hline ENSG00000266709 & -0.92347604 & $1.49 \mathrm{E}-15$ & $1.2 \mathrm{E}-14$ & AC005224.4 \\
\hline ENSG00000269927 & -0.941179574 & 0.0000116 & 0.0000356 & AC004817.3 \\
\hline ENSG00000224080 & -0.957073973 & 0.00000138 & 0.00000479 & UBE2FP1 \\
\hline ENSG00000171119 & -0.732024185 & 0.000859632 & 0.001973741 & NRTN \\
\hline ENSG00000182795 & -0.713257 & 0.000893473 & 0.002045361 & C1orf116 \\
\hline ENSG00000235859 & -0.981085776 & $1.34 \mathrm{E}-11$ & $7.99 \mathrm{E}-11$ & AC006978.1 \\
\hline ENSG00000184058 & -0.781722778 & 0.000947799 & 0.002160685 & TBX1 \\
\hline ENSG00000137959 & -0.589383242 & 0.000982869 & 0.00223288 & IFI44L \\
\hline ENSG00000249485 & -0.994236206 & 0.0000105 & 0.0000326 & RBBP4P1 \\
\hline ENSG00000188848 & -0.612794187 & 0.00106899 & 0.00241599 & BEND4 \\
\hline ENSG00000235180 & -1.024177912 & $1.2 \mathrm{E}-09$ & $5.92 \mathrm{E}-09$ & LINC00601 \\
\hline ENSG00000280123 & -1.03782143 & $2.74 \mathrm{E}-12$ & $1.73 \mathrm{E}-11$ & AC023632.6 \\
\hline
\end{tabular}


Table 3 (continued)

\begin{tabular}{|c|c|c|c|c|}
\hline Locus & $\log F C$ & $P$-Value & FDR & Gene name \\
\hline ENSG00000176912 & -1.038065676 & $3.47 \mathrm{E}-10$ & $1.79 \mathrm{E}-09$ & TYMSOS \\
\hline ENSG00000134317 & -0.746307481 & 0.001354336 & 0.003003002 & GRHL1 \\
\hline ENSG00000130208 & -0.676215853 & 0.001374168 & 0.003039733 & APOC1 \\
\hline ENSG00000250462 & -1.061344837 & $4.22 \mathrm{E}-32$ & $8.64 \mathrm{E}-31$ & LRRC37BP1 \\
\hline ENSG00000155465 & -0.67282726 & 0.001515904 & 0.00333088 & SLC7A7 \\
\hline ENSG00000230316 & -1.096386598 & $3.09 \mathrm{E}-10$ & $1.61 \mathrm{E}-09$ & FEZF1-AS1 \\
\hline ENSG00000115318 & -0.601269693 & 0.001614401 & 0.003523135 & LOXL3 \\
\hline ENSG00000125931 & -0.665048797 & 0.001714986 & 0.003725307 & CITED1 \\
\hline ENSG00000261534 & -1.102227861 & $7.9 \mathrm{E}-10$ & $3.95 \mathrm{E}-09$ & AL596244.1 \\
\hline ENSG00000214719 & -1.106140664 & $1.01 \mathrm{E}-16$ & $8.81 \mathrm{E}-16$ & AC005562.1 \\
\hline ENSG00000234456 & -1.116449494 & $4.03 \mathrm{E}-22$ & $4.92 \mathrm{E}-21$ & MAGI2-AS3 \\
\hline ENSG00000092200 & -0.685034084 & 0.001895898 & 0.004088527 & RPGRIP1 \\
\hline ENSG00000216775 & -1.169568862 & $1.42 \mathrm{E}-39$ & $4.14 \mathrm{E}-38$ & AL109918.1 \\
\hline ENSG00000260686 & -1.172038199 & 0.000000482 & 0.00000176 & AC008669.1 \\
\hline ENSG00000226943 & -1.177930323 & $1.53 \mathrm{E}-08$ & $6.66 \mathrm{E}-08$ & ALG1L5P \\
\hline ENSG00000188185 & -1.185262956 & 0.000001 & 0.00000354 & LINC00265 \\
\hline ENSG00000113389 & -0.597567416 & 0.002456821 & 0.005192422 & NPR3 \\
\hline ENSG00000248890 & -1.205696789 & $6.08 \mathrm{E}-18$ & $5.82 \mathrm{E}-17$ & HHIP-AS1 \\
\hline ENSG00000170270 & -0.68387924 & 0.002558896 & 0.005392531 & GON7 \\
\hline ENSG00000119508 & -0.711455187 & 0.002680663 & 0.005624765 & NR4A3 \\
\hline ENSG00000140905 & -0.636524948 & 0.002992587 & 0.006224563 & GCSH \\
\hline ENSG00000050730 & -0.636431758 & 0.003037792 & 0.006309593 & TNIP3 \\
\hline ENSG00000170989 & -0.64441633 & 0.003150496 & 0.006522018 & S1PR1 \\
\hline ENSG00000256304 & -1.233661403 & $2.46 \mathrm{E}-13$ & $1.68 \mathrm{E}-12$ & CCDC150P1 \\
\hline ENSG00000237424 & -1.291854855 & $1.19 \mathrm{E}-11$ & $7.12 \mathrm{E}-11$ & FOXD2-AS1 \\
\hline ENSG00000227359 & -1.292403358 & 0.000000113 & 0.000000447 & AC017074.1 \\
\hline ENSG00000230615 & -1.294100524 & $2.92 \mathrm{E}-32$ & $6.03 \mathrm{E}-31$ & AL139220.2 \\
\hline ENSG00000244300 & -1.311706541 & $6.67 \mathrm{E}-08$ & 0.000000271 & GATA2-AS1 \\
\hline ENSG00000183762 & -0.599097723 & 0.004686398 & 0.009396411 & KREMEN1 \\
\hline ENSG00000168405 & -1.328267416 & $4.82 \mathrm{E}-09$ & $2.23 \mathrm{E}-08$ & СМАНР \\
\hline ENSG00000144834 & -0.620458505 & 0.004968065 & 0.00989472 & TAGLN3 \\
\hline ENSG00000279519 & -1.3382021 & $3.67 \mathrm{E}-12$ & $2.3 \mathrm{E}-11$ & AC007382.1 \\
\hline ENSG00000105371 & -0.591348772 & 0.00761156 & 0.014575965 & ICAM4 \\
\hline ENSG00000128536 & -0.58264386 & 0.007717241 & 0.014763271 & CDHR3 \\
\hline ENSG00000226806 & -1.402676685 & 0.000000047 & 0.000000194 & AC011893.1 \\
\hline ENSG00000257732 & -1.507011758 & $2.49 \mathrm{E}-14$ & $1.83 \mathrm{E}-13$ & AC089983.1 \\
\hline
\end{tabular}

$50 \mathrm{mM}$ sodium bisulfite, $10 \mathrm{mM} \mathrm{MgCl}_{2}, 1 \%$ Triton $\mathrm{X}-100$, $8.6 \%$ sucrose, and $10 \mathrm{mM}$ sodium butyrate). Working at $4{ }^{\circ} \mathrm{C}$, pellets were then purified by three rounds of centrifugation $(16,000 \times g$ for $15 \mathrm{~s})$ and resuspension by vortexing, in lysis buffer for the first two rounds and washing buffer (10 mM Tris pH 7.4, $13 \mathrm{mM}$ EDTA) for the final round; supernatant fractions were discarded. The obtained pellets containing chromatin were resuspended in $0.4 \mathrm{~N}$ sulfuric acid, the mixture was incubated at $4{ }^{\circ} \mathrm{C}$ for $1 \mathrm{~h}$ and then centrifuged at $16,000 \times g$ for $10 \mathrm{~min}$, and the histone- containing supernatants were kept. Samples were incubated with acetone (1:9) to block the acid overnight at $-20^{\circ} \mathrm{C}$ and then centrifuged at $16,000 \times g$ for $10 \mathrm{~min}$ histone pellets were air dried for $5 \mathrm{~min}$ and then resuspended in water for analysis. Cell extracts of PDX samples were obtained by disrupting tissue using a pellet pestle (Sigma; Z359947) with lysis buffer $(50 \mathrm{mM}$ Tris- $\mathrm{HCl} \mathrm{pH} 8,10 \mathrm{mM}$ EDTA, $1 \%$ SDS, and $1 \mathrm{mM}$ DTT). Proteins were separated by SDSpolyacrylamide gel electrophoresis gel and analyzed with the indicated antibodies. 
Fig. 5 LOXL2 suppresses sensitivity in TNBC cells. a Cell viability was measured at different timepoints using an MTT assay of cultured different breast cancer cells and PDXs. The effects of doxorubicin, TSA, and doxorubicin plus TSA were analyzed. b Dissociated cells from PDX-549 were orthotopically implanted into NOD/SCID mice and injected intraperitoneally twice weekly with TSA $(0.25 \mathrm{mg} / \mathrm{kg})$, doxorubicin $(2 \mathrm{mg} / \mathrm{kg}$ mouse weight), or doxorubicin plus TSA. Tumor volumes were measured twice a week and are given as averages. Results are given as averages of six independent tumors \pm SEM
A

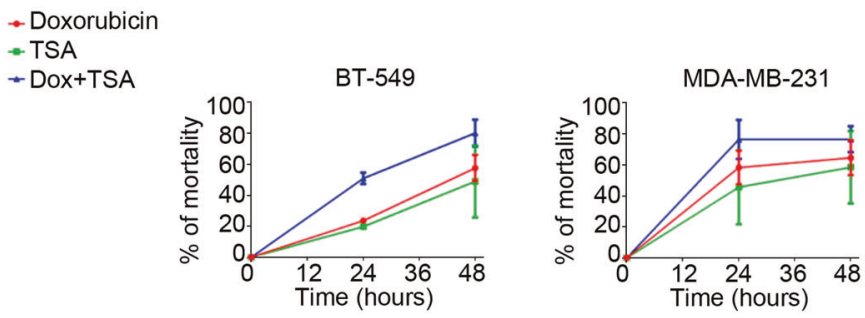

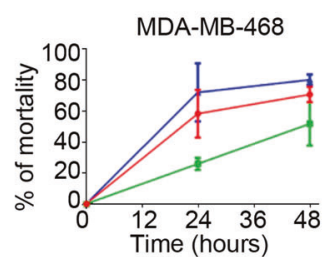

PDX-154
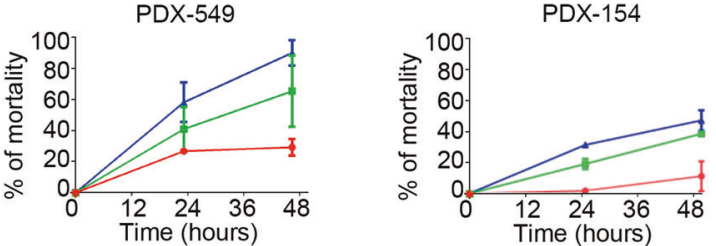

B

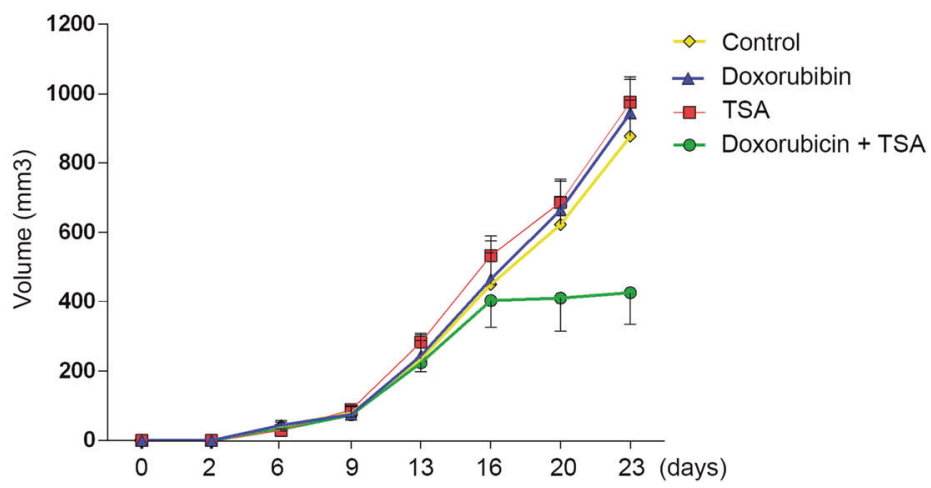

\section{Recombinant LOXL2 purification}

LOXL2-encoding baculovirus were amplified, and LOXL2Flag recombinant proteins (wt and mutant) were produced in Sf9 cells according to standard procedures [3]. Cell lysis was performed as previously described [54]. Cell extracts were incubated with Flag M2 beads for $4 \mathrm{~h}$ at $4{ }^{\circ} \mathrm{C}$ and then washed $4 \times$ times with $20 \mathrm{mM}$ HEPES $\mathrm{pH} 7.4,1 \mathrm{mM}$ $\mathrm{MgCl}_{2}, 300 \mathrm{mM} \mathrm{NaCl}, 10 \mathrm{mM} \mathrm{KCl}, 10 \%$ glycerol, and $0.2 \%$ Triton X-100. Recombinant proteins were eluted with the Flag peptide $(1 \mu \mathrm{g} / \mu \mathrm{l})$ for $1 \mathrm{~h}$ at $4{ }^{\circ} \mathrm{C}$.

\section{Oxidation reaction}

Nucleosomes $(2 \mu \mathrm{g})$ were incubated with recombinant LOXL2 purified protein in oxidation buffer, $20 \mathrm{mM}$ HEPES $\mathrm{pH} 7.4,100 \mathrm{mM} \mathrm{NaCl}, 1 \mathrm{mM} \mathrm{MgCl}_{2}$, and $1 \mathrm{mM}$ DTT. Reactions were carried out for the indicated times at $37^{\circ} \mathrm{C}$ and then analyzed by SDS/PAGE and western blotting. To detect the aldehyde group, biotin hydrazide (BTH) $(5 \mathrm{mM})$ was added after the oxidation reaction for $2 \mathrm{~h}$ at $25^{\circ} \mathrm{C}$. Finally, biotinylated histones were immunoprecipitated with streptavidin beads, and oxidized $\mathrm{H} 3$ was detected by western blot.

\section{Dot blot assay}

For dot blot assays, $1 \mu \mathrm{g}$ of each peptide (in $10 \mu \mathrm{l}$ of sample) was applied under low vacuum to a prewetted nitrocellulose membrane (Amersham Protran 0.45 nitrocellulose, GE Healthcare) using a dot blot apparatus (HYBRI-DOT Manifold; Life Technologies). After blocking the entire blot in $15 \mathrm{~mL}$ of $5 \%$ nonfat dry milk and $0.1 \%$ Tween-20 Trisbuffered saline for $1 \mathrm{~h}$ at room temperature, the blot was probed with the indicated antibodies.

\section{ChIP experiments}

For ChIP experiments, cells were first crosslinked in $1 \%$ formaldehyde for $10 \mathrm{~min}$ at $37^{\circ} \mathrm{C}$. Crosslinking was stopped by adding glycine to a final concentration of $0.125 \mathrm{M}$ for $2 \mathrm{~min}$ at room temperature. Cell monolayers were scraped in cold soft-lysis buffer $(50 \mathrm{mM}$ Tris $\mathrm{pH} 8.0$, $10 \mathrm{mM}$ EDTA, $0.1 \%$ NP-40, and $10 \%$ glycerol), and incubated $20 \mathrm{~min}$ on ice. Nuclei pellets were lysed with SDS lysis buffer (1\% SDS, $10 \mathrm{mM}$ EDTA, $50 \mathrm{mM}$ Tris $\mathrm{pH}$ 8.0 ), and extracts were sonicated to generate 200-1500-bp DNA fragments. For immunoprecipitation, supernatants were diluted 1:10 with dilution buffer, and samples were 
incubated with rotation overnight at $4{ }^{\circ} \mathrm{C}$ with primary antibody or irrelevant immunoglobulin G. Samples were then treated with elution buffer $\left(100 \mathrm{mM} \mathrm{Na}_{2} \mathrm{CO}_{3}\right.$ and $1 \%$ SDS) for $1 \mathrm{~h}$ at $37^{\circ} \mathrm{C}$ and incubated at $65^{\circ} \mathrm{C}$ overnight after addition of $\mathrm{NaCl}$ to a final concentration of $200 \mathrm{mM}$, to reverse formaldehyde crosslinking. After proteinase $\mathrm{K}$ treatment for $1 \mathrm{~h}$ at $55^{\circ} \mathrm{C}$, DNA was purified with MinElute PCR purification kit (Qiagen; 28006) and eluted in Milli-Q water. Genomic regions (GRs) were detected by quantitative staining with PCR SYBR Green (Quantabio; 95073), and the ChIP results were quantified relative to the input amount and the amount of $\mathrm{H} 3$ immunoprecipitated in each condition.

Peaks of H3K4ox were called from sequence reads detected through ChIP-seq using the MACS2 tool [26]. The chromatin state files for HepG2 and HMEC cells were computed by the ENCODE project using the ChromHMM tool [27] from https://genome.ucsc.edu/cgi-bin/hgFileUi? $\mathrm{db}=\mathrm{hg} 19 \& \mathrm{~g}=$ wgEncodeBroadHmm. Statistical overrepresentation of H3K4ox peaks detected by ChIP-seq was assessed from the two cell lines across several chromatin states: heterochromatin, repressed, insulator, strong enhancer (sum of states 4 and 5), poised/weak enhancer (sum of states 6 and 7), promoter (sum of states 1 and 2), and poised promoter. The contingency table of the Fisher's test carried out for this contained the number of nucleotides within peaks, chromatin states, intersections thereof, and the remaining portion of the genome (computed as the difference from the effective genome size for ChIP-seq peaks calling). The same procedure was applied to detect H3K4ox peak overrepresentation in lamin-associated domains of chromatin, obtained from [55]. ChIP-PCR used the following GR number and peak positions: GR\#1: chr 14 (79003315-79007307); GR\#2: chr 5 (151967551-151971 984); GR\#3: chr 1 (230657019-230658589); GR\#4: chr 2 (41741709-41745780); GR\#5: chr 17 (53951879-5395 5135); GR\#6: chr 4 (62611008-62615505); GR\#7: chr 6 (67550237-67554488); GR\#8: chr 5 (66894215-668990 21); and GR\#9: chr 13 (89334968-89336774). The sequences of the primers used can be found in Supplementary Table 2.

\section{ATAC-seq and ATAC-qPCR experiments}

ATAC experiments were performed as described previously [28]. Cells were harvested and treated with transposase Tn5 (Nextera DNA Library Preparation Kit, Illumina; FC-121-1030). DNA was purified using MinElute PCR purification kit (Qiagen), samples were amplified by PCR using NEBNext High-Fidelity $2 \times$ PCR Master Mix (New England Biolabs; M5041), and DNA was again purified with the MinElute PCR purification kit. Reads produced by ATAC sequencing of two control
(Control) replicates and two LOXL2 KD sequencing replicates (LOXL2 KD) were aligned to the hg 19 build of the reference human genome using Bowtie 2 [56] with default parameters for pair-end sequencing. ATAC peaks were then called by combining aligned reads of both replicates of the control and the KD using MACS2. To allow for false discovery rate (FDR) threshold selection further downstream in the analysis, no FDR restrictions were imposed on the ATAC peak calling. For ATACqPCR experiments, the final elution product of the ATAC protocol was diluted 1:50. Incorporation of the transposase Tn5 to GRs was detected by quantitative staining with PCR SYBR Green (Quantabio; 95073). ATACqPCR results for the selected GRs were normalized to the same unaffected GR (HPRT promoter) for each condition. The sequences of the primers used can be found in Supplementary Table 2 .

\section{RNA-seq analysis}

Reads produced by RNA-seq of the same replicates as described above of two controls (Control) and two LOXL2 KDs were aligned to the $\mathrm{hg} 19$ build of the reference human transcriptome using TopHat2 [57] with default parameters for pair-end sequencing. Aligned reads were then analyzed using a standard Cufflinks pipeline [58] to detect differentially expressed genes between the two conditions ( $L O X L 2$ $\mathrm{KD}$ and Control).

\section{Differential expression analysis of transposable elements (TEs)}

The trimmed RNA-seq reads from the two controls and the two LOXL2 samples were processed with the TEtools program [59] using the hg19 annotation of TEs. The obtained count table was then processed with NOIseq [60] to perform a differential expression analysis of LOXL2 against the control. Expression was normalized using the TMM method, whereby TEs with a probability higher than 0.95 of being differentially expressed were considered as statistically significant.

\section{Replicate correlation}

The read count (coverage) at each position of the hg19 human reference genome was computed for each replicate of the H3K4ox ChIP-seq and the control and LOXL2 KD ATAC sequencing, using the BEDtools genomecov capability [61]. Genomic positions with zero-read counts were filtered out. Replicate files of each experiment were merged to produce a single file aligned by genomic position, and the corresponding Pearson's correlation coefficient of read counts was computed. For the graphical representation of 
the correlation, 100,000 genomic positions were randomly selected.

\section{Analysis of ATAC peaks that overlap with H3K4ox peaks}

All significant $\left(P<10^{-5}\right)$ ATAC peaks (LOXL2 KD versus control) and H3K4ox peaks were first intersected with the BEDtools intersect program [61]. Based on this intersection, ATAC peaks were classified as overlapping (if they intersected an H3K4ox peak) or orphan (if not). Only intersections involving more than $95 \%$ of the sequence of ATAC peaks were considered. Control and LOXL2 KD read counts over all genomic positions (see above) were intersected with both overlapping and orphan peaks. Read counts over genomic positions of control and experiment replicates were averaged. To carry out the heatmap representation, peak sequences (overlapping or orphan) were aligned by their summits. For linear representation, the average experimental read counts at each downstream and upstream position were summed for both the experimental and the control counts. Position-wise sums were then divided by the read count sum value obtained for the summit of control read counts, thus making all sums relative to the maximum control value.

\section{Integrated analysis of H3K40x and ATAC peaks, and differentially expressed genes}

The differentially expressed genes detected through the RNA-seq analysis of control and LOXL2 KD cells were selected if they were in close vicinity (either up- or downstream) to overlapping ATAC peaks. Two different distance thresholds $(0.5$ and $1 \mathrm{Mb})$ were used to detect close differentially expressed genes.

\section{Inhibition of RNA synthesis, ATM kinase, and cell cycle analysis}

MDA-MB-231-infected cells selected for $48 \mathrm{~h}$ with puromycin $(2.5 \mu \mathrm{g} / \mathrm{ml})$ were seeded and then maintained under puromycin selection.

For inhibition of RNA synthesis, cells were treated at $48 \mathrm{~h}$ with $200 \mu \mathrm{M}$ cordycepin (Sigma; C3394) and fixed with $100 \%$ cold ethanol at designated timepoints.

For inhibition of the ATM kinase, cells were incubated at the 24-h timepoint with KU55933 (Sigma; SML1109) for a further $24 \mathrm{~h}$ at two different concentrations (either 5 or $10 \mu \mathrm{M})$, or for $2 \mathrm{~h}$ with doxorubicin $(1 \mu \mathrm{M})$ as a positive control.

For cell cycle analysis, cells at $48 \mathrm{~h}$ after puromycin selection were first synchronized through the double thymidine block protocol. Specifically, cells were seeded to
$50 \%$ confluency, incubated for $14 \mathrm{~h}$ with complete growth medium (supplemented with $2 \mathrm{mM}$ thymidine), washed $2 \times$ with phosphate-buffered saline (PBS), and released by a 9-h incubation with complete medium growth. Cells were washed again $2 \times$ with PBS, incubated $14-\mathrm{h}$ with $2-\mathrm{mM}$ thymidine, and released with complete growth medium. Cells were then harvested at the designated timepoints and fixed with $100 \%$ cold ethanol. After two days, fixed cells were stained with propidium iodide (PI) and analyzed by flow cytometry using BD FACSCalibur (Becton Dickinson). Results were analyzed using BD CellQuest Pro software.

\section{Non-replicative cell experiment}

MDA-MB-231 cells were seeded in coverslips and maintained during all the experiment in Dulbecco's modified Eagle's medium (Biowest; L0106-500) with $0.5 \%$ fetal bovine serum (Gibco; 10270106) at $37{ }^{\circ} \mathrm{C}$ in $5 \% \mathrm{CO}_{2}$. After $24 \mathrm{~h}$, cells were infected with lentiviral particles for $L O X L 2$ KD. After $96 \mathrm{~h}$ under selection, cells were fixed with paraformaldehyde $4 \%$ with PBS.

\section{Comet assay}

MDA-MB-231-infected cells under puromycin selection (see above) were seeded at the 48-h timepoint and grown another $48 \mathrm{~h}$ more (still with puromycin at $2.5 \mu \mathrm{g} / \mathrm{ml}$ ). DNA strand breaks were measured at the single-cell level for MDA-MB-231 control or LOXL2 KD cells using an alkaline comet assay with the CometAssay kit (Trevigen, Gaithersburg, MD) following the manufacturer's instructions. Briefly, 5000 cells in $50 \mu \mathrm{l}$ PBS were combined with $500 \mu \mathrm{l}$ molten LMAgarose (at $37^{\circ} \mathrm{C}$ ), and $50 \mu \mathrm{l}$ of this was immediately transferred to a CometSlide. After a 10-min incubation at $4{ }^{\circ} \mathrm{C}$, slides were immersed in $4{ }^{\circ} \mathrm{C}$ lysis solution and incubated overnight at $4{ }^{\circ} \mathrm{C}$. Slides were then immersed in freshly prepared alkaline solution $(200 \mathrm{mM}$ $\mathrm{NaOH}, 1 \mathrm{mM}$ EDTA $\mathrm{pH}>13$ ) for $1 \mathrm{~h}$ at $4{ }^{\circ} \mathrm{C}$ in the dark. For electrophoresis, slides were placed in an electrophoresis slide tray containing alkaline solution and incubated with voltage $(21 \mathrm{~V})$ for $30 \mathrm{~min}$. After that, slides were immersed twice in $\mathrm{H}_{2} \mathrm{O}$ for $5 \mathrm{~min}$ and then once in $70 \%$ ethanol for $5 \mathrm{~min}$. Samples were dried at $37^{\circ} \mathrm{C}$ and stained with SYBR Green staining solution (SYBR Green I, Invitrogen) using a 1:10000 dilution in TE $(10 \mathrm{mM}$ Tris- $\mathrm{HCl} \mathrm{pH} 7.5,1 \mathrm{mM}$ EDTA) for 30 min Finally, slides were dried at $37^{\circ} \mathrm{C}$, and cells were imaged using an Olympus BX61 microscope.

\section{Rescue experiments}

MDA-MB-231-infected cells under puromycin selection (see above) were seeded at the 48-h timepoint, incubated 
under puromycin selection for an addition $24 \mathrm{~h}$, and then transfected with the SUV-39H1-EGFP vector or reinfected with retroviral particles for LOXL2-FLAG or lentiviral particles for $\mathrm{H} 1$ expression. After $24 \mathrm{~h}$, cells were fixed for immunofluorescence.

\section{Immunofluorescence, image acquisition, and analysis}

Cells were fixed with $4 \%$ paraformaldehid for $15 \mathrm{~min}$ at room temperature, blocked for $1 \mathrm{~h}$ with $1 \%$ PBS-bovine serum albumin, incubated at room temperature for $2 \mathrm{~h}$ with primary antibody, washed $3 \times$ with PBS, and then incubated for $1 \mathrm{~h}$ at room temperature with the secondary antibody. Cells were washed again $3 \times$ with PBS, incubated for 5 min with $4^{\prime}, 6-$ diamidino-2-phenylindole (DAPI) $(0.25 \mathrm{mg} / \mathrm{ml})$ for cell nuclei staining, and then mounted with fluoromount. Fluorescence images corresponding to DAPI, GFP, $\gamma$-H2AX and 53BP1 were acquired in a Leica TCS SPE microscope using a Leica DFC300 FX camera and the Leica IM50 software.

\section{Metaphase spreads}

For metaphase spread preparations, cells were treated with colcemid $(0.1 \mathrm{ug} / \mathrm{ml})$ for $4 \mathrm{~h}$. Cells were trypsinized, hypotonically swollen in $0.075 \mathrm{M} \mathrm{KCl}$ for $15 \mathrm{~min}$ at $37^{\circ} \mathrm{C}$, and then fixed (75\% $\mathrm{MeOH}$ and $25 \%$ acetic acid, ice cold). Metaphase preparations were spread on glass slides, stained with $10 \%$ Giemsa stain (Sigma), and mounted in DPX mounting medium (PanReac). Images were taken using a Leica DM6000 microscope (Leica, Wetzlar, Germany) and analyzed using Fiji Software (https://fiji.sc/).

\section{Cellular viability experiment}

Breast cancer cell lines and PDX cells were seeded in 96-well plates. Cellular viability was analyzed using Thiazolyl Blue Tetrazolium Bromide (MTT) (Sigma; M5655) at different timepoints. The concentrations used were: $0.1 \mu \mathrm{M}$ doxorubicin for all samples; 250-nM TSA for the breast cancer cell lines; and 500-nM TSA for the PDXs cells. Absorbance was detected at $565 \mathrm{~nm}$ on Infinite ${ }^{\circledR} 200$ PRO Series Multimode Reader (Tecan Group Ltd.) and analyzed with i-control ${ }^{\mathrm{TM}}$ Microplate Reader Software (Tecan Group Ltd.)

\section{Cancer PDXs and treatments}

Samples from patients with breast cancer were obtained from the operating room and transferred to the pathology department, where breast cancer samples were collected, transferred to the animal facility, and implanted into mice, to generate PDXs. All samples were implanted within 60 to 90 min after surgical removal. All patients willingly signed an informed consent, and the study was approved by the Ethics Committee of the Vall d'Hebron Hospital.

For in vivo experiments, a tumor from an established PDX was dissociated into single cells by enzymatic digestion (collagenase at $300 \mathrm{U} / \mathrm{ml}$ and hyaluronidase at $200 \mathrm{U} /$ $\mathrm{ml}$ ) during $1 \mathrm{~h}$ at $37^{\circ} \mathrm{C}$ on a rotating wheel. The solution of digested tumor was treated with $0.025 \%$ trypsin and filtered sequentially using 100 and $40 \mu \mathrm{m}$ strainers. Isolated cells were plated in culture dishes with DMEM-F12 supplemented with FBS, glutamine, and penicillin/streptomycin. Once the culture was established, $10^{6}$ cells were injected into the number four fat pad of 6-week old NOD. CB17PrkdcSCID/J (NOD/SCID) female mice (Charles River) with Matrigel. For this, mice were anesthetized and shaved, the fourth and fifth sets of nipples were localized, and an inverted $\mathrm{Y}$ incision was made from the midline point between the fourth set of nipples, ending between the fourth and fifth sets to expose the fourth and fifth fat pads on one side. After the injection, animals were sutured, and analgesics injected. Animals were kept in a clean cage with drinking water supplemented with $1 \mu \mathrm{M} 17-\beta$-estradiol. Tumor xenografts were measured with callipers every 3 days, and tumor volume was determined using the formula: (length $\times$ width $\left.^{2}\right) \times(\mathrm{pi} / 6)$. At the end of the experiment, animals were anesthetized with a $1.5 \%$ isoflorane-air mixture and were killed by cervical dislocation.

Treatments were administered intraperitoneally twice weekly. One week after cell injection, mice were randomized and treated with TSA $(0.25 \mathrm{mg} / \mathrm{kg})$, doxorubicin ( $2 \mathrm{mg} / \mathrm{kg}$ mouse weight), or doxorubicin plus TSA. The control group was injected with sterile PBS.

Mice were maintained and treated in accordance with institutional guidelines of Vall d'Hebron University Hospital Care and Use Committee.

\section{Data availability}

\section{GSE96064.}

Acknowledgements We would like to thank R. Peña and J. Valle for technical assistance, V.A. Raker for manuscript editing, G. Gil for manuscript reading and advice, A. Jordan for H1-GFP constructs, T. Jenuwein for SUV-39 constructs, and H. Galvez-Garcia for ATAC protocol implementation. This work was supported by grants from Instituto de Salud Carlos III (ISCIII) FIS/FEDER (PI12/01250; CP08/ 00223; PI16/00253; and CB16/12/00449), MINECO (SAF201348849-C2-1-R) to SP, BFU2015-68354 to THS, Breast Cancer Research Foundation (BCRF-17-008) to JA, AGL2014-52395-C2-2-R to DA, Worldwide Cancer Research, Red Temática de Investigación Cooperativa en Cáncer (RD012/0036/005), Fundación Científica de la Asociación Española contra el Cáncer, and Fundació La Marató TV3. THS was supported by institutional funding (MINECO) through the Centres of Excellence Severo Ochoa award and the CERCA Programme of the Catalan Government, and SS-B, by a Fundació La Caixa fellowship. We thank La Caixa Foundation and Cellex Foundation for provide research facilities and equipment. GV has received 
funding from the MINECO (a "Juan de la Cierva Incorporation" fellowship; IJCI-2014-20723). SP was a recipient of a Miguel Servet contract (ISCIII/FIS), and AI, JPC-C, LP-G, and GS-B are supported by contracts from Worldwide Cancer Research, Fundació La Marató TV3, Fundació FERO, and a FI Fellowship from the Generalitat de Catalunya, respectively.

\section{Compliance with ethical standards}

Conflict of interest The authors declare that they have no conflict of interest.

Publisher's note: Springer Nature remains neutral with regard to jurisdictional claims in published maps and institutional affiliations.

Open Access This article is licensed under a Creative Commons Attribution 4.0 International License, which permits use, sharing, adaptation, distribution and reproduction in any medium or format, as long as you give appropriate credit to the original author(s) and the source, provide a link to the Creative Commons license, and indicate if changes were made. The images or other third party material in this article are included in the article's Creative Commons license, unless indicated otherwise in a credit line to the material. If material is not included in the article's Creative Commons license and your intended use is not permitted by statutory regulation or exceeds the permitted use, you will need to obtain permission directly from the copyright holder. To view a copy of this license, visit http://creativecommons. org/licenses/by/4.0/.

\section{References}

1. Bannister AJ, Kouzarides T. Regulation of chromatin by histone modifications. Cell Res. 2011;21:381-95.

2. Williamson PR, Kagan HM. Reaction pathway of bovine aortic lysyl oxidase. J Biol Chem. 1986;261:9477-82.

3. Herranz N, Dave N, Millanes-Romero A, Pascual-Reguant L, Morey L, Diaz VM, et al. Lysyl oxidasE-like 2 (LOXL2) oxidizes trimethylated lysine 4 in histone H3. FEBS J. 2016;283:4263-73.

4. Iturbide A, Garcia de Herreros A, Peiro S. A new role for LOX and LOXL2 proteins in transcription regulation. FEBS J. 2015;282:1768-73.

5. Iturbide A, Pascual-Reguant L, Fargas L, Cebria JP, Alsina B, Garcia de Herreros A, et al. LOXL2 oxidizes methylated TAF10 and controls TFIID-dependent genes during neural progenitor differentiation. Mol Cell. 2015;58:755-66.

6. Millanes-Romero A, Herranz N, Perrera V, Iturbide A, LoubatCasanovas J, Gil J, et al. Regulation of heterochromatin transcription by Snail1/LOXL2 during epithelial-to-mesenchymal transition. Mol Cell. 2013;52:746-57.

7. Barker HE, Cox TR, Erler JT. The rationale for targeting the LOX family in cancer. Nat Rev Cancer. 2012;12:540-52.

8. Cano A, Santamaria PG, Moreno-Bueno G. LOXL2 in epithelial cell plasticity and tumor progression. Future Oncol. 2012;8:1095-108.

9. Ahn SG, Dong SM, Oshima A, Kim WH, Lee HM, Lee SA, et al. LOXL2 expression is associated with invasiveness and negatively influences survival in breast cancer patients. Breast Cancer Res Treat. 2013;141:89-99.

10. Almendro V, Cheng YK, Randles A, Itzkovitz S, Marusyk A, Ametller E, et al. Inference of tumor evolution during chemotherapy by computational modeling and in situ analysis of genetic and phenotypic cellular diversity. Cell Rep. 2014;6:514-27.

11. De Craene B, Berx G. Regulatory networks defining EMT during cancer initiation and progression. Nat Rev Cancer. 2013;13:97-110.
12. Escriva M, Peiro S, Herranz N, Villagrasa P, Dave N, MontserratSentis B, et al. Repression of PTEN phosphatase by Snaill transcriptional factor during gamma radiation-induced apoptosis. Mol Cell Biol. 2008;28:1528-40.

13. Vega S, Morales AV, Ocana OH, Valdes F, Fabregat I, Nieto MA. Snail blocks the cell cycle and confers resistance to cell death. Genes Dev. 2004;18:1131-43.

14. McDonald OG, Wu H, Timp W, Doi A, Feinberg AP. Genomescale epigenetic reprogramming during epithelial-to-mesenchymal transition. Nat Struct Mol Biol. 2011;18:867-74.

15. Peinado H, Moreno-Bueno G, Hardisson D, Perez-Gomez E, Santos V, Mendiola M, et al. Lysyl oxidase-like 2 as a new poor prognosis marker of squamous cell carcinomas. Cancer Res. 2008;68:4541-50.

16. Herranz N, Pasini D, Diaz VM, Franci C, Gutierrez A, Dave N, et al. Polycomb complex 2 is required for E-cadherin repression by the Snail1 transcription factor. Mol Cell Biol. 2008;28:4772-81.

17. Bekker-Jensen S, Lukas C, Kitagawa R, Melander F, Kastan MB, Bartek J, et al. Spatial organization of the mammalian genome surveillance machinery in response to DNA strand breaks. J Cell Biol. 2006;173:195-206.

18. Soutoglou E, Misteli T. Activation of the cellular DNA damage response in the absence of DNA lesions. Science. 2008;320:1507-10.

19. Bonilla CY, Melo JA, Toczyski DP. Colocalization of sensors is sufficient to activate the DNA damage checkpoint in the absence of damage. Mol Cell. 2008;30:267-76.

20. Gursoy-Yuzugullu O, House N, Price BD. Patching broken DNA: nucleosome dynamics and the repair of DNA breaks. J Mol Biol. 2016;428:1846-60.

21. Ziv Y, Bielopolski D, Galanty Y, Lukas C, Taya Y, Schultz DC, et al. Chromatin relaxation in response to DNA double-strand breaks is modulated by a novel ATM- and KAP-1 dependent pathway. Nat Cell Biol. 2006;8:870-6.

22. Burgess RC, Burman B, Kruhlak MJ, Misteli T. Activation of DNA damage response signaling by condensed chromatin. Cell Rep. 2014;9:1703-17.

23. Ayrapetov MK, Gursoy-Yuzugullu O, Xu C, Xu Y, Price BD. DNA double-strand breaks promote methylation of histone $\mathrm{H} 3$ on lysine 9 and transient formation of repressive chromatin. Proc Natl Acad Sci USA. 2014;111:9169-74.

24. Khurana S, Kruhlak MJ, Kim J, Tran AD, Liu J, Nyswaner K, et al. A macrohistone variant links dynamic chromatin compaction to BRCA1-dependent genome maintenance. Cell Rep. 2014;8:1049-62.

25. Holliday DL, Speirs V. Choosing the right cell line for breast cancer research. Breast Cancer Res. 2011;13:215.

26. Zhang Y, Liu T, Meyer CA, Eeckhoute J, Johnson DS, Bernstein $\mathrm{BE}$, et al. Model-based analysis of ChIP-Seq (MACS). Genome Biol. 2008;9:R137.

27. Ernst J, Kellis M. ChromHMM: automating chromatin-state discovery and characterization. Nat Methods. 2012;9:215-6.

28. Buenrostro JD, Giresi PG, Zaba LC, Chang HY, Greenleaf WJ. Transposition of native chromatin for fast and sensitive epigenomic profiling of open chromatin, DNA-binding proteins and nucleosome position. Nat Methods. 2013;10:1213-8.

29. Tsompana M, Buck MJ. Chromatin accessibility: a window into the genome. Epigenetics Chromatin. 2014;7:33.

30. Burgess RC, Misteli T. Not all DDRs are created equal: noncanonical DNA damage responses. Cell. 2015;162:944-7.

31. Bayona-Feliu A, Casas-Lamesa A, Reina O, Bernues J, Azorin F. Linker histone $\mathrm{H} 1$ prevents R-loop accumulation and genome instability in heterochromatin. Nat Commun. 2017;8:283.

32. Tuduri S, Crabbe L, Conti C, Tourriere H, HoltgrevE-Grez H, Jauch A, et al. Topoisomerase I suppresses genomic instability by preventing interference between replication and transcription. Nat Cell Biol. 2009;11:1315-24. 
33. Garcia-Rubio ML, Perez-Calero C, Barroso SI, Tumini E, Herrera-Moyano E, Rosado IV, et al. The Fanconi anemia pathway protects genome integrity from R-loops. PLoS Genet. 2015;11:e1005674.

34. Gan W, Guan Z, Liu J, Gui T, Shen K, Manley JL, et al. R-loopmediated genomic instability is caused by impairment of replication fork progression. Genes Dev. 2011;25:2041-56.

35. Bakkenist CJ, Kastan MB. DNA damage activates ATM through intermolecular autophosphorylation and dimer dissociation. Nature. 2003;421:499-506.

36. Kaidi A, Jackson SP. KAT5 tyrosine phosphorylation couples chromatin sensing to ATM signalling. Nature. 2013;498:70-4.

37. Yoshida M, Kijima M, Akita M, Beppu T. Potent and specific inhibition of mammalian histone deacetylase both in vivo and in vitro by trichostatin A. J Biol Chem. 1990;265:17174-9.

38. Toth KF, Knoch TA, Wachsmuth M, Frank-Stohr M, Stohr M, Bacher $\mathrm{CP}$, et al. Trichostatin A-induced histone acetylation causes decondensation of interphase chromatin. J Cell Sci. 2004;117:4277-87.

39. Peinado H, Del Carmen Iglesias-de la Cruz M, Olmeda D, Csiszar $\mathrm{K}$, Fong KS, Vega S, et al. A molecular role for lysyl oxidasE - like 2 enzyme in snail regulation and tumor progression. EMBO J. 2005;24:3446-58.

40. Schietke R, Warnecke C, Wacker I, Schodel J, Mole DR, Campean $\mathrm{V}$, et al. The lysyl oxidases LOX and LOXL2 are necessary and sufficient to repress E-cadherin in hypoxia: insights into cellular transformation processes mediated by HIF-1. J Biol Chem. 2010;285:6658-69.

41. Voloshenyuk TG, Landesman ES, Khoutorova E, Hart AD, Gardner JD. Induction of cardiac fibroblast lysyl oxidase by TGFbeta1 requires PI3K/Akt, Smad3, and MAPK signaling. CytoAbsorbance was detected atkine. 2011;55:90-7.

42. Fischer KR, Durrans A, Lee S, Sheng J, Li F, Wong ST, et al. Epithelial-to-mesenchymal transition is not required for lung metastasis but contributes to chemoresistance. Nature. 2015;527:472-6.

43. Zheng X, Carstens JL, Kim J, Scheible M, Kaye J, Sugimoto H, et al. Epithelial-to-mesenchymal transition is dispensable for metastasis but induces chemoresistance in pancreatic cancer. Nature. 2015;527:525-30.

44. Baldeyron C, Soria G, Roche D, Cook AJ, Almouzni G. HP1alpha recruitment to DNA damage by p150CAF-1 promotes homologous recombination repair. J Cell Biol. 2011;193:81-95.

45. Soria G, Polo SE, Almouzni G. Prime, repair, restore: the active role of chromatin in the DNA damage response. Mol Cell. 2012;46:722-34.

46. Murga M, Jaco I, Fan Y, Soria R, Martinez-Pastor B, Cuadrado $\mathrm{M}$, et al. Global chromatin compaction limits the strength of the DNA damage response. J Cell Biol. 2007;178:1101-8.

47. Goodarzi AA, Noon AT, Deckbar D, Ziv Y, Shiloh Y, Lobrich M, et al. ATM signaling facilitates repair of DNA double-strand breaks associated with heterochromatin. Mol Cell. 2008;31:167-77.
48. Fong SF, Dietzsch E, Fong KS, Hollosi P, Asuncion L, He Q, et al. Lysyl oxidase-like 2 expression is increased in colon and esophageal tumors and associated with less differentiated colon tumors. Genes Chromosomes Cancer. 2007;46:644-55.

49. Moreno-Bueno G, Salvador F, Martin A, Floristan A, Cuevas EP, Santos V, et al. Lysyl oxidase-like 2 (LOXL2), a new regulator of cell polarity required for metastatic dissemination of basal-like breast carcinomas. EMBO Mol Med. 2011;3:528-44.

50. Torres S, Garcia-Palmero I, Herrera M, Bartolome RA, Pena C, Fernandez-Acenero MJ, et al. LOXL2 is highly expressed in cancer-associated fibroblasts and associates to poor colon cancer survival. Clin Cancer Res. 2015;21:4892-902.

51. Wong CC, Tse AP, Huang YP, Zhu YT, Chiu DK, Lai RK, et al. Lysyl oxidase-like 2 is critical to tumor microenvironment and metastatic niche formation in hepatocellular carcinoma. Hepatology. 2014;60:1645-58.

52. Martin A, Salvador F, Moreno-Bueno G, Floristan A, RuizHerguido C, Cuevas EP, et al. Lysyl oxidase-like 2 represses Notch1 expression in the skin to promote squamous cell carcinoma progression. EMBO J. 2015;34:1090-109.

53. Bao S, Wu Q, McLendon RE, Hao Y, Shi Q, Hjelmeland AB, et al. Glioma stem cells promote radioresistance by preferential activation of the DNA damage response. Nature. 2006;444:756-60.

54. Wu M, Wang PF, Lee JS, Martin-Brown S, Florens L, Washburn $\mathrm{M}$, et al. Molecular regulation of $\mathrm{H} 3 \mathrm{~K} 4$ trimethylation by Wdr82, a component of human Set1/COMPASS. Mol Cell Biol. 2008;28:7337-44.

55. Guelen L, Pagie L, Brasset E, Meuleman W, Faza MB, Talhout $\mathrm{W}$, et al. Domain organization of human chromosomes revealed by mapping of nuclear lamina interactions. Nature. 2008;453:948-51.

56. Langmead B, Trapnell C, Pop M, Salzberg SL. Ultrafast and memory-efficient alignment of short DNA sequences to the human genome. Genome Biol. 2009;10:R25.

57. Kim D, Pertea G, Trapnell C, Pimentel H, Kelley R, Salzberg SL. TopHat2: accurate alignment of transcriptomes in the presence of insertions, deletions and gene fusions. Genome Biol. 2013;14:R36.

58. Trapnell C, Roberts A, Goff L, Pertea G, Kim D, Kelley DR, et al. Differential gene and transcript expression analysis of RNA-seq experiments with TopHat and Cufflinks. Nat Protoc. 2012;7:562-78.

59. Lerat E, Fablet M, Modolo L, Lopez-Maestre H, Vieira C. TEtools facilitates big data expression analysis of transposable elements and reveals an antagonism between their activity and that of piRNA genes. Nucleic Acids Res. 2017;45:e17.

60. Tarazona S, Furio-Tari P, Turra D, Pietro AD, Nueda MJ, Ferrer A, et al. Data quality aware analysis of differential expression in RNA-seq with NOISeq R/Bioc package. Nucleic Acids Res. 2015;43:e140.

61. Quinlan AR, Hall IM. BEDTools: a flexible suite of utilities for comparing genomic features. Bioinformatics. 2010;26:841-2. 